\title{
Engineering Scale Development of the Vapor-Liquid-Solid (VLS) Process for the Production of Silicon Carbide Fibrils 2 ? 7 ? \\ OSTI
}

April 1995

Phase II Report Prepared by

R. W. Ohnsorg, W. E. Hollar, Jr., and S. K. Lau

The Carborundum Company

Technology Division

P.O. Box 832

Niagara Falls, NY 14302

F. K. Ko and K. Schatz

Advanced Product Development

2500 Pearl Buck Road

Bristol, PA 19007

under

Subcontract Number 62X-SD861C

for

OAK RIDGE NATIONAL LABORATORY

Oak Ridge, Tennessee 37831

managed by

MARTIN MARIETTA ENERGY SYSTEMS, INC.

for the

U.S. Department of Energy

under Contract No. DE-AC05-84OR21400 
This report has been reproduced directly from the best available copy.

Available to DOE and DOE contractors from the Office of Scientific and Technical Information, P.O. Box 62, Oak Ridge, TN 37831; prices available from (615) 576-8401, FTS 626-8401.

Available to the public from the National TEchnical Information Service, U.S. Department of Commerce, 5285 Port Royal Rd., Springfield, VA 22161.

This report was prepared as an account of work sponsored by an agency of the United States Government. Neither the United States Government nor any agency thereof, nor any of their employees, makes any warranty, expressed or implied, or assumes any legal liability or responsibility for the accuracy, completeness, or usefulness of any information, apparatus, product, or process disclosed, or represents that its use would not infringe privately owned rights. Reference herein to any specific commercial product, process, or service by trade name, trademark, manufacturer, or otherwise, does not necessarily constitute or imply its endorsement, recommendation, or favoring by the United States Government or any agency thereof. The views and opinions of authors expressed herein do not necessarily state or reflect those of the United States Government or any agency thereof. 


\section{Engineering Scale Development of the Vapor-Liquid-Solid (VLS) Process for the Production of Silicon Carbide Fibrils}

April 1995

Research Sponsored by the U.S. Department of Energy, Fossil Energy Advanced Research and Technology Development Materials Program, DOE/FE AA 151010 0, Work Breakdown Structure Element CARB-5

Phase II Report Prepared by

R. W. Ohnsorg, W. E. Hollar, Jr., and S. K. Lau

The Carborundum Company

Technology Division

P.O. Box 832

Niagara Falls, NY 14302

F. K. Ko and K. Schatz

Advanced Product Development

2500 Pearl Buck Road

Bristol, PA 19007

under

Subcontract Number 62X-SD861C

for

OAK RIDGE NATIONAL LABORATORY

Oak Ridge, Tennessee 37831

managed by

MARTIN MARIETTA ENERGY SYSTEMS, INC.

for the

U.S. Department of Energy

under Contract No. DE-AC05-84OR21400 

Table of Contents

1. Abstract 1

2. Introduction 1

3. Phase I Summary 2

3.1 Phase I Description and Results 2

4. Phase II Program 4

4.1 Phase II Goals 4

4.2 Feasibility and Demonstration of Yarn Fabrication 5

4.3 Composite Fabrication $\quad 7$

4.4 Economic Analysis $\quad 7$

$\begin{array}{ll}\text { 4.4.1 Summary } & 7\end{array}$

$\begin{array}{ll}\text { 4.4.2 Background } & 7\end{array}$

4.4.3 Economic Analysis Procedure $\quad 13$

4.4.4 Economic Analysis Results 13

5. Acknowledgments 18

6. References 19

Figures

1. SiC Fibril Process Flow Chart 8

2. SiC Fibril Synthesis Reaction Mechanisms $\quad 10$

3. Carborundum SiC Fibril Reactor 11

4. Los Alamos Whisker-Growing Furnace 11

5. Effect of Reactor Position on Reactant Partial Pressures and SiC Fibril

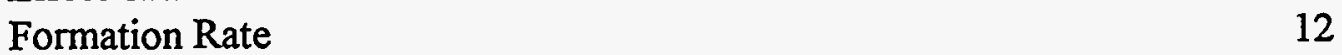

6. Reactor Design Evolution $\quad 12$

7. Production Cost $(\$ / / \mathrm{b})$ Sensitivities 17

Tables

1. Number of Furnace Units Required 14

2. Fibril Production Cost Summary (I) 15

3. Fibril Production Cost Summary (II) 16

Appendix I Conversion of SiC Fibrils to Linear, Aligned Fibrous Assemblies for Ceramic Matrix Composites (APD Report)

Appendix IIA Economic Analysis Conservative Approach

Appendix IIB Economic Analysis Aggressive Approach 



\section{Engineering Scala Development of the Vapor-Liquid-Solid (VLS) Process \\ for the Production of Silicon Carbide Fibrils \\ Phase II Report}

\section{Abstract}

As reinforcements for composites, VLS SiC fibrils have attractive mechanical properties including high strength, high modulus, and excellent creep resistance. To make use of their excellent mechanical properties in a composite, a significant volume fraction $(>10 \%)$ of aligned, long fibrils ( $>2 \mathrm{~mm}$ ) needs to be consolidated in the ceramic matrix. The fibrils must be processed into an assembly that will allow for composite fabrication while maintaining fibril alignment and length. With Advanced Product Development (APD) as the yarn fabrication subcontractor, Carborundum investigated several approaches to achieve this goal, including traditional yarn-forming processes such as carding and airvortex spinning and nontraditional processes such as tape forming and wet casting. Carborundum additionally performed an economic analysis for producing 500 and 10,000 pounds of SiC fibrils annually using both conservative and more aggressive processing parameters. With the aggressive approach, the projected costs for SiC fibril production for 500 and 10,000 pounds per year are $\$ 1,340$ /pound and $\$ 340 /$ pound, respectively.

\section{Introduction}

Vapor-liquid-solid (VLS) SiC fibrils are high-aspect-ratio, fine-diameter, single-crystal SiC fibers which have attractive mechanical, physical, and thermal properties for use as reinforcements in ceramic matrix composite (CMC) material systems. These properties include: high-temperature stability; unmatched tensue strength, modulus, and creep resistance; and good oxidation resistance.

It is the objective of this program to demonstrate the technical and economic feasibility of scaling the VLS SiC fibril process; to demonstrate the feasibility of producing linear, aligned fibril preforms for ceramic matrix composites (CMCs); and to determine the subsequent performance of $\mathrm{SiC}$ fibrils as a reinforcement for CMCs. Incorporated in this report is a summary recapping the earlier Phase I effort and the goals and accomplishments of the current Phase II program, including a detailed report by APD and an economic analysis for producing 500 and 10,000 pounds of harvested and beneficiated $\mathrm{SiC}$ fibrils annually. 


\section{Phase I Summary}

Carborundum completed a Phase I program in early 1993 with the following program tasks:

- Design a prototype scale reactor capable of producing 100 to $500 \mathrm{lb} /$ year of VLS SiC fibrils

- Improve process productivity through increases in growth rate and yield

- Demonstrate a process gas recycle system

- Develop a harvesting/beneficiation/leaching process

- Demonstrate characterization techniques

\subsection{Phase I Description and Results}

Reactor designs and processing parameters such as process gas flow rate and composition, degree of mixing, and temperature were the key factors to the growth rate of the fibrils. The Los Alamos National Laboratory (LANL) contributions during the 1980s included increased understanding of several key process variables such as gas mixing, catalystsubstrate interactions, and Si reactant supply on the growth process[1,2]. An order-ofmagnitude increase in growth rates was realized and post-processing steps and fibril characterization techniques were developed[1,3].

Carborundum's efforts then focused on transferring the LANL technology. A reactor with a height twice that (12" vs. 6 ") of the LANL reactor was constructed to evaluate reactor scaleup feasibility. This configuration allowed evaluation of growth process limitations caused by reactant concentration variations with reactor height. The effect of SiO and $\mathrm{CH}_{4}$ supply rates on the fibril growth rate was evaluated and variations in reactant supply with reactor position were identified as the controlling scaleup parameters. Modification of the synthesis process conditions was found necessary to achieve uniform fibril growth in a taller reactor.

The results of this work showed that a prototype scale reactor was possible. Evaluation of the relationship between reactant supply and the fibril growth process led to an improved understanding of reactor design requirements culminating in the development of a computer model capable of simulating reactor performance as a function of process conditions and reactor geometry. The kinetic data obtained in the development reactor was incorporated into the model to simulate the effects of critical process variables such as temperature and reactant supply within a single reactor channel.

Acceptable process gas mixing was difficult to achieve due to the laminar flow conditions. LANL pioneered the application of "turbulent jet mixing" and this, along with improved gas distribution, allowed for impressive increases in growth rates and product uniformity in the LANL reactor[4]. However, turbulent jet mixing had several potential drawbacks in a taller reactor since it depended on the interaction of the SiO generators with the process gas inlet jets and the relatively high-pressure drops required[5]. 
Carborundum addressed these concerns with oscillatory flow mixing[2]. It was based on the observation that improved heat and mass transfer characteristics in laminar flow situations can occur when periodic flow perturbations result in the presence of a flow destabilizing g,ometry. In this case the $\mathrm{SiO}$ generators served as the flow-destabilizing structures. A flow oscillator was developed based on a mechanical bellows to produce periodic perturbations in the process gas flow.

The remainder of Phase I focused on evaluating process specifiations and the demonstration of a process gas recycle system. The cost of hydrogen which comprises $\sim 80 \%$ of the process gas can become a controlling production cost factor in the absence of such a system[2]. In addition, higher process gas flow rates than in the LANL reactor were necessary to sustain fibril growth rates in scaleup reactors[1].

An external recycle system was designed, fabricated, and installed. This included cooling, filtering, and recompressing the exit gas and adjusting the inlet gas composition to replace missing or off-spec components. A fixed fraction of the process gas was removed to maintain a steady gas composition and monitored using a gas chromatograph (CG) and $\mathrm{CO}$ gas analyzer. Growth experiments confirmed that $\mathrm{SiC}$ fibrils can be grown using recycled process gas and preliminary evaluation indicated that both the fibril growth rate and product characteristics were similar to the baseline process without a recycle system[2].

Fibril postprocessing requirements depended on the fibril characteristics required for the composite application. Growth substrate harvesting and liquid beneficiation techniques were developed to maximize fibril yield and dispersion for subsequent processing. Chemical leaching was developed to remove residual metal catalyst without visible degradation of the fibril surfaces. Postprocessing yield was extremely inportant since manufacturing costs were inversely related to yield.

Fibril characterization was an important component of process development and scaleup. The primary focus haci been on physical ciraracterization by microscopy to study product morphology and dimensions. Since systhesis process conditions strongly affected fibril physical characteristics, characterization was necessary to determine tradeoffs between process productivity and product characteristics. A quantitative technique was developed for measuring and correlating fibril diameter distributions[2] which would serve in achieving improved or tailored diameter distributions for specific applications. Mechanical strength evaluation was performed by single fiber composite testing. These experiments confirmed the high strength of VLS SiC fibrils reported elsewhere. 
A critical conclusion that came out of Phase I was that fibril manufacturing costs depended on the fibril product specification as defined by the end-use application and the composite fabrication route. The following summarizes the key Phase I accomplishments:

- Vertical reactor scaleup feasibility demonstrated

- Increased understanding of the relationship between reactant supply and fibril growth rate and product characteristics

- Nucleation and growth phases identified

- Computer simulation model developed consistent with experimental observation of process variable effects

- Process gas rucycle system demonstrated

- Postprocessing demonstrated

\section{Phase II Program}

Upon the successful completion of the Phase I effort, Carborundum initiated the Phase II program in July 1993.

\subsection{Phase II Goals}

Ceramic matrix composites containing moderate amounts of long, aligned $\mathrm{SiC}$ fibrils have the potential to behave like a continuous fiber reinforced composite. Because of their high strength, a relatively low loading of fibrils would produce composite behavior. Successful demonstration of this predicted behavior could make SiC fibrils an attractive candidate for many CMC applications. To achieve this result, a process for aligning long $(>2 \mathrm{~mm})$ fibrils in a preform and consolidating them into a fully-dense composite must be demonstrated. Thus, a process for fabricating linear, aligned assemblies of fibrils is a crucial step in making fibrils a serious CMC reinforcement candidate. This requirement resulted in Phase II of the program.

The specific Phase II goals included the following:

- Develop a process capable of converting SiC fibrils into continuous, textile-like, linear fibrous assemblies

- Produce demonstration samples for composite fabrication experiments

- Complete an economic analysis of the process

The Phase II program requires demonstration of the feasibility of a scalable process for orienting fibrils and fabricating them into yarns. As a result, Carborundum processed a large quantity of fibrils and supplied them in three different forms (air-harvested product, harvested and beneficiated product, and as-synthesized growth substrates) to the subtier contractor, Advanced Product Development (APD), for evaluation in yarn-forming experiments. Both traditional and nontraditional processes for forming $\mathrm{SiC}$ fibrils into linear, aligned assemblies were evaluated for composite applications. Carborundum also conducted an economic analysis for producing 500 and 10,000 pounds of $\mathrm{SiC}$ fibrils annually. 


\subsection{Feasibility and Demonstration of Yarn Fabrication (APD Subcontract)}

The unique properties of $\mathrm{SiC}$ fibrils make forming them into yarns or other aligned assemblies very challenging. Fibrous ceramic materials are more difficult to form into textile assemblies than typical fibers such as cotton, wool, and synthetics because ceramic fibers tend to be stiffer, shorter, smoother, and finer in diameter. All of these properties make disentangling, alignment, and incorporation into a yarn mnre difficult. SiC fibrils are especially challenging due to their relatively short length and high stiffness.

All processes for forming yarns or other linear assemblies involve similar processing steps. The fibrils are typically collected in the form of tangled mats by harvesting them from growth substrates. For conversion to a yarn, this mat must be opened and aligned. The loose, aligned assembly is then attenuated into a linear assembly. Next a technique like spinning is used to integrate the aligned assembly into a structure with acceptable mechanical properties.

Yarn-forming processes can be categorized as traditional and nontraditional. The traditional process of forming cotton-based yarns consists of carding which disentangles and aligns the fiber mat followed by attenuation into a sliver which is essentially an aligned assembly with minimal physical coherence. Spinning then applies twist to the fibers to entangle them which gives the yarn its mechanical strength for handling and processing by braiding, weaving, etc.

Carding is a high-speed textile process with well-developed technology. The fibers are first aligned and disentangled to open up the structure prior to yarn formation. Carrier textile fibers such as cotton can be used to facilitate processing of the $\mathrm{SiC}$ fibrils. The success of the carding process depends on the card clothing configuration. The card clothing consists of materials ranging from metal to cloth with a variable number of points per unit area. The combination of the card material and point density determines the effectiveness of the carding process. Hand carding was chosen since it made rapid screening of different card clothing possible.

Air-vortex spinning is a yarn-forming process in which fibers are continuously fed into a vacuum-formed air vortex which catches and entangles them around the rotating free end of the previously-formed yarn. Unlike carding, 1) use of air is more gentle on the fibrils than mechanical features, 2) intermediate drafting is eliminated, and 3) reduced twisting for entanglement results in less fibril damage.

Traditional and modified traditional fiber processing resulted in substantial damage to the fibers. To be successful, the yarn-forming process needs to be gentle and all handling steps should be minimized. An alternative to carding as the alignment process is necessary and the assembly needs to be consolidated using techniques other than twisting. 
Fiber separation, alignment, and integration can also be achieved by nontraditional (textile) processes such as the adhesive-tape method and the wet-laid method. Both address the major issues associated with the traditional process. Both use less aggressive techniques to align the fibrils and polymeric materials (carriers) to provide the mechanical strength necessary for fabrication of the assemblies into preforms.

In the adhesive-tape method, adhesive tape is used to harvest the fibrils directly from the growth substrate. Separation and alignment can be accomplished by lightly brushing the fibrils in one direction on the growth plate. Adhesive tape (3/4" wide) is then pressed onto the fibrils which break at the plate surface and stick to the tape in a linear alignment. The catalyst balls are removed by leaching. The tape with the adherent fibrils can then be further processed into a 2D or 3D assembly by weaving, braiding, etc.

Advantages of this process include:

- High degree of bending

- Utilization of all sizes of fibrils

- Less fibril handling

- Reduced fibril damage

- Tape contributes to tensile strength

- Tape protects fibrils from subsequent processing damage and loss

However, disadvantages include:

- Low fibril/tape ratio

- Tape/adhesive must be removed

- Fibril orientation is not optimized

- Catalyst balls are not removed

In the wet-laid method fibril separation is achieved by dispersing the beneficiated fibrils in an aqueous solution containing appropriate binders and dispersants. The fibril dispersion is then passed through a tube which causes fibril alignment followed by deposition into a narrow channel on a moving, porous plate. The aligned fibrils fill the channel as the plate is moved and the liquid phase is removed by a vacuum applied to the base of the channel. The binder holds the fibrils together or the fibrils can be overwrapped with a polymerbased yarn.

In this process:

- Long and short fibrils can be used

- Harvested fibrils can be used

- There is good alignment

- There is less damage to fibrils from liquid alignment than by mechanical means 
The various yarn-forming processes described above were evaluated at APD. A summary of the accomplishments is listed below:

- The processibility of $\mathrm{SiC}$ fibrils by traditional textile processes was critically evaluated.

- Key processing parameters for conversion of fibrils to linear, aligned assemblies were identified.

- Woven tape samples to demonstrate product form feasibility were produced.

- A fluid-based process for producing aligned fibril assemblies was successfully demustrated.

- Linear, aligned fibril assemblies for CMC test specimen fabrication were produced.

A more detailed discussion of VLS SiC fibril textile processing is given in APD's attached report (Appendix I).

\subsection{Composite Fabrication}

A total of $20 \mathrm{~g}$ of harvested and beneficiated $\mathrm{SiC}$ fibrils was processed by APD into linear, aligned assemblies via the wet-laid method. Two preforms for CMC fabrication were prepared by laying up (0/90) these yarns in two $1.75^{\prime \prime}$ diameter $\times 0.50$ " high graphite CVI crucibles supplied by Oak Ridge National Laboratory (ORNL). The preform density was increased by compaction. These preforms were delivered to ORNL for subsequent CVI CMC fabrication. Another preform using $10 \mathrm{~g}$ of randomly oriented fibrils was also prepared in a third CVI crucible and sent to ORNL for CMC fabrication.

\subsection{Economic Analysis}

\subsubsection{Summary}

The production of vapor-liquid-solid (VLS) SiC fibrils has been estimated to cost $\$ 2,200 / \mathrm{lb}$ and $\$ 550 / \mathrm{lb}$ in quantities of 500 and $10,000 \mathrm{lb} /$ year, respectively, with current operating $(0.85)$ and quality yield $(0.55)$ factors. By increasing the operating factor to 0.90 and the quality yield factor to 0.85 , the $\$ / \mathrm{b}$ can be reduced to $\$ 1,350$ and $\$ 340$ for 500 and $10,000 \mathrm{lb} /$ year, respectively.

\subsubsection{Background}

The production of $\mathrm{SiC}$ fibrils is a transport and reaction process in which $\mathrm{SiO}, \mathrm{CH}_{4}$, and $\mathrm{H}_{2}$ process gases react at high temperatures to grow the $\mathrm{SiC}$ fibrils on a catalyst-doped (Fe-Si-C) growth substrate.

The SiC fibril manufacturing process flow diagram is shown in Fig.1. The heart of the process involves the preparation of the $\mathrm{SiO}$ generators and the fibril growth substrates. To prepare the generators, insulating refractory ceramic brick sections are dipped in a 


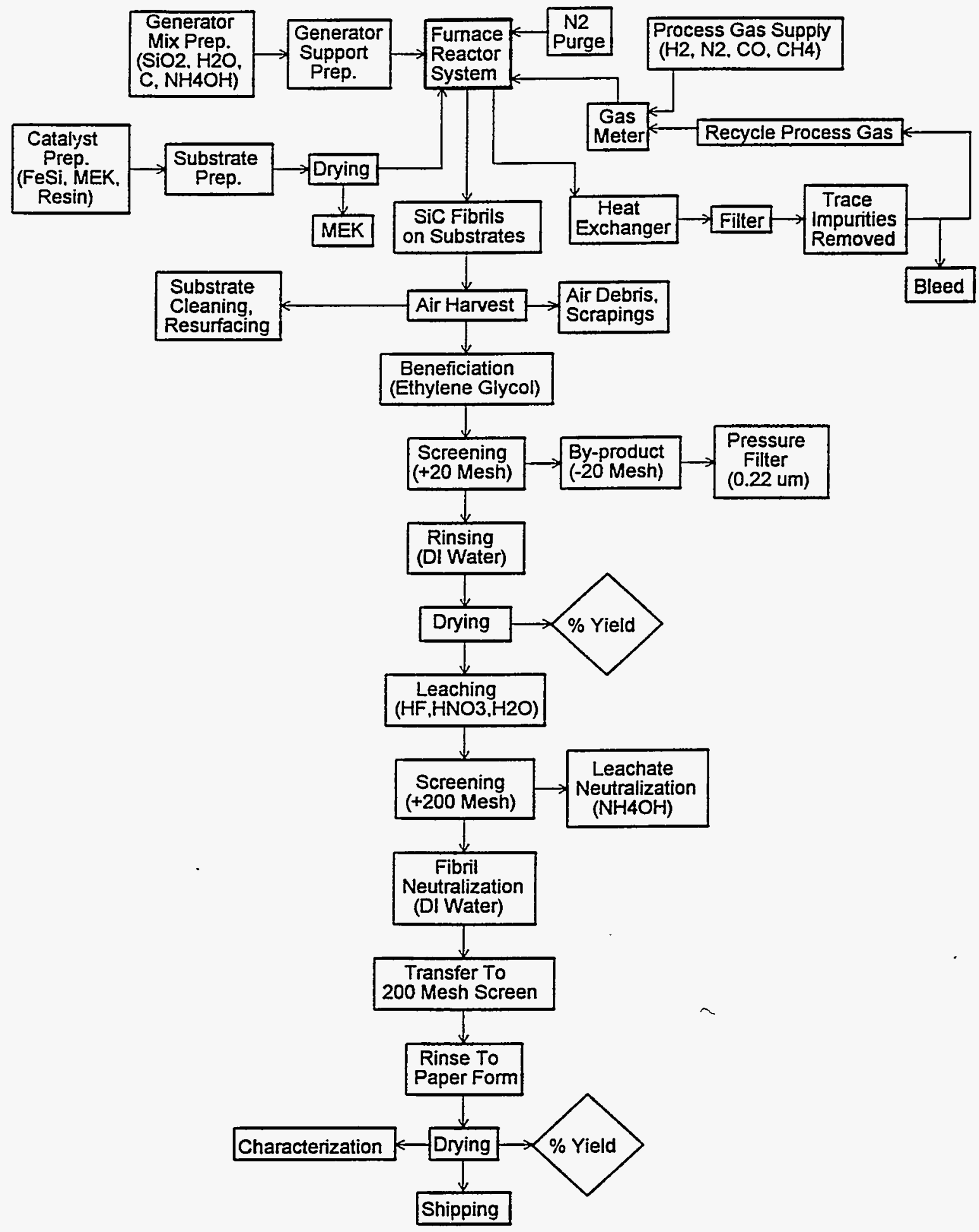

Figure 1 SiC Fibril Process Flow Chart 
slurry of carbon black and colloidal silica. SiO gas is formed in-situ at these generators via the carbothermal reduction of silica by carbon according to the reaction:

$$
\mathrm{C}(\mathrm{s})+\mathrm{SiO}_{2}(\mathrm{~s})=\mathrm{SiO}(\mathrm{g})+\mathrm{CO}(\mathrm{g})
$$

To prepare the fibril growth substrates, graphite plates are coated with a FeSi catalyst dispersed in a solution of MEK and resin. The growth of fibrils by the VLS process is promoted and controlled by the liquid FeSi catalyst droplets. To start the fibril growth reaction, process gas containing $\mathrm{H}_{2} / \mathrm{N}_{2} / \mathrm{CO} / \mathrm{CH}_{4}$ is introduced at the base of the reactor and flows in vertically rectangular channels. Through a series of reactions depicted schematically in Fig. 2, SiC fibrils are formed.

The reactor currently used at Carborundum for producing $\mathrm{SiC}$ fibrils is a vertical reactor with dimensions of 6" $\times 6 "$ " 12 " (Fig. 3). The Los Alamos National Laboratory (LANL) reactor (Fig. 4) which was used to develop process concepts during the 1980 s, on the other hand, is a horizontal design with the same dimensions. These two reactors have approximately the same volume and growth substrate area.

It had been determined in Phase I of this program that fibril growth characteristics are position dependent. The $\mathrm{SiO}$ generator weight loss was at a maximum near the center of the reactor and decreased dramatically with increasing height. This behavior was probably the result of both mass transfer and temperature effects. The effect of reactor position on reactant partial pressures and $\mathrm{SiC}$ fibril growth rate is shown in Fig. 5.

A computer simulation model was developed as a tool for compiling scaleup data in a form suitable for reactor design and process development. Its effectiveness for predicting the fibril growth rate as a function of position and synthesis process conditions was demonstrated. The model successfully predicted trends in the fibril growth r..te as a function of reactor height with changing process conditions. As a result, temperature, process gas flow rate, and $\mathrm{CH}_{4}$ partial pressure could be varied to determine how best to obtain a uniform $\mathrm{SiC}$ fibril growth pattern. 


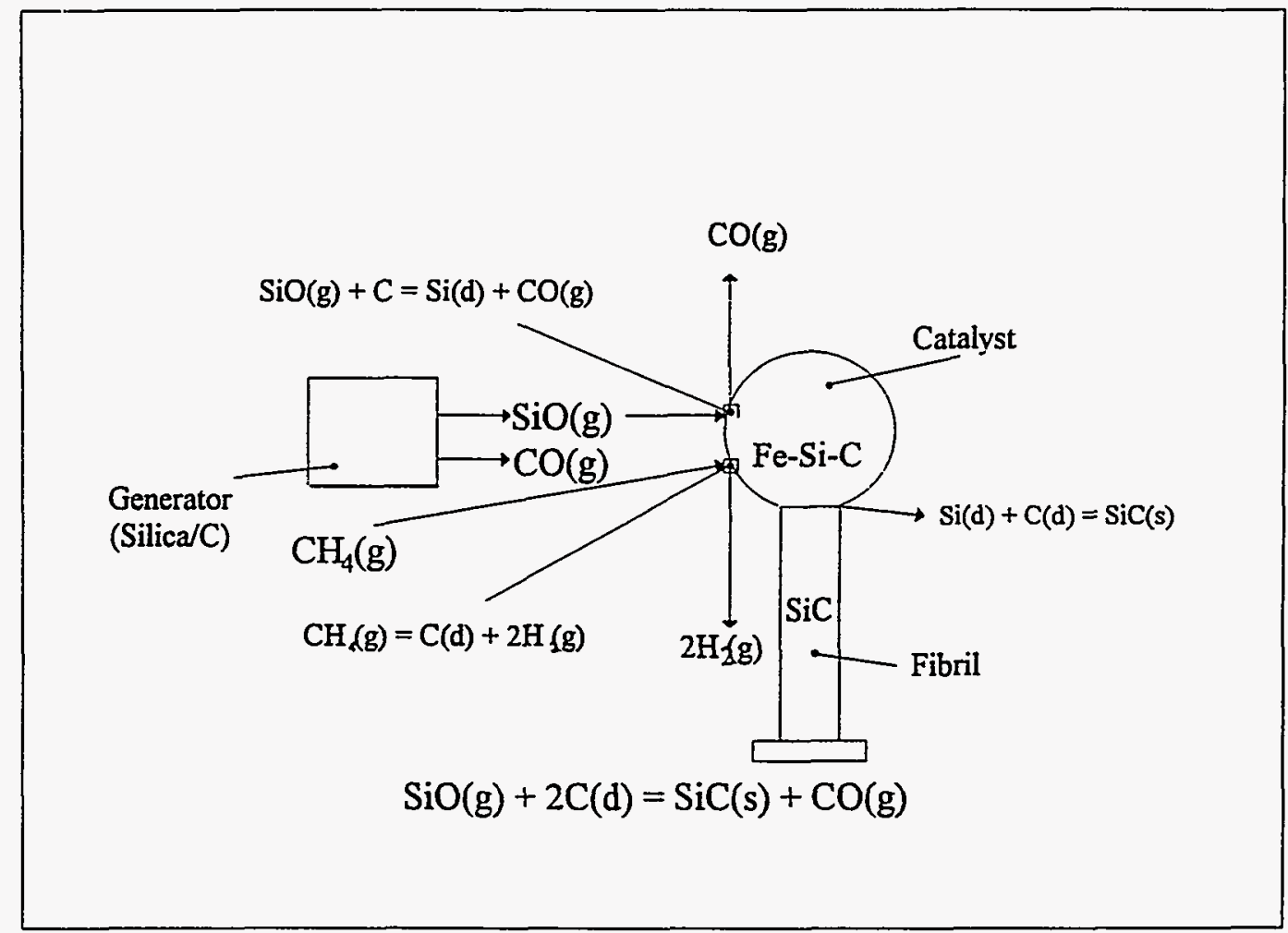

Figuri 2 SiC Fibril Synthesis Reaction Mechanisms

Based on this simulation model, four examples of reactor designs with their corresponding growth substrate areas and predicted yields are shown in Fig. 6 for the 6 " $\times 6$ " $\times 12$ " laboratory reactor. The Generation 1 reactor is the current design where process gases flow vertically upward in the four channels indicated. A higher and more uniform product yield can be achieved with additional process gas inputs in the reactor sides for increased reactor efficiency as shown in Generation 2a. Doubling the growth substrates doubles the total growth surface area and, despite the shorter fibril length, increases product yield due to the larger number of fibrils that grow to "maturity" and their larger diameter (Generation 2b). A final design for significantly increasing the growth surface is to use graphite rods instead of plates as growth substrates (Generation 2c). 


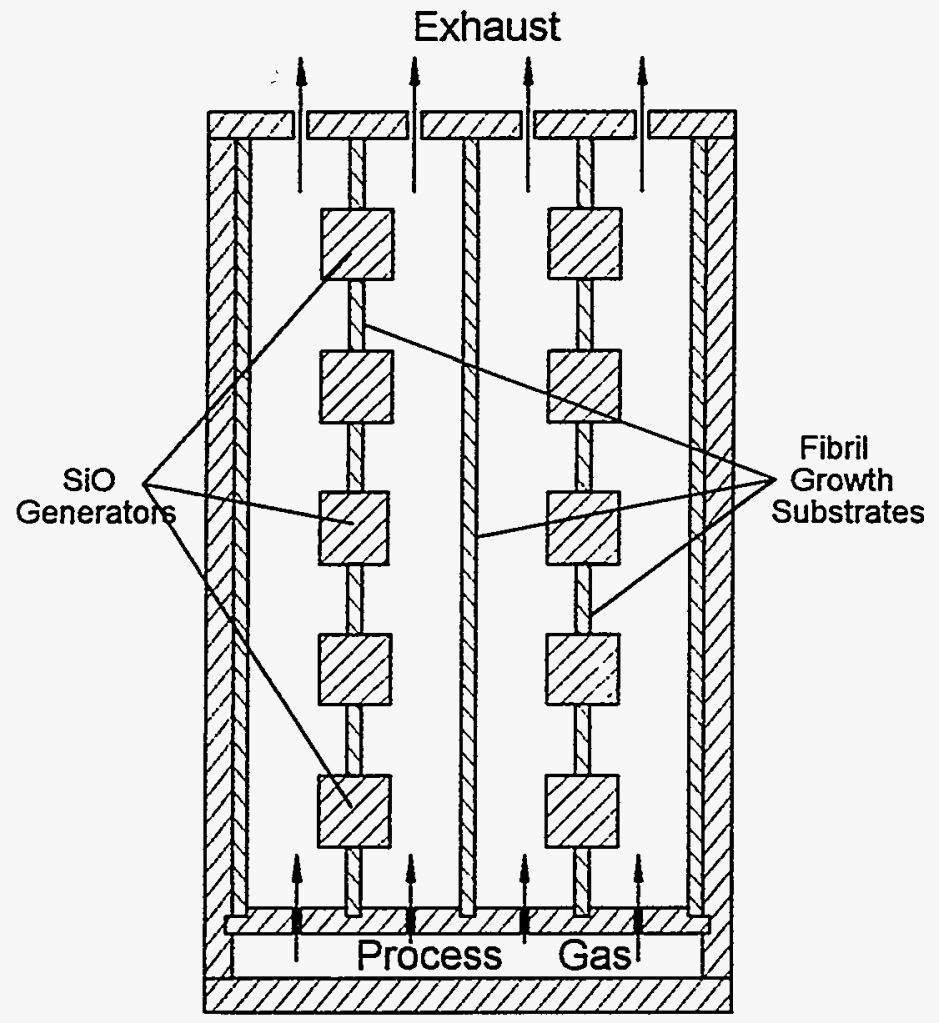

Figure 3 Carborundum SiC Fibril Reactor

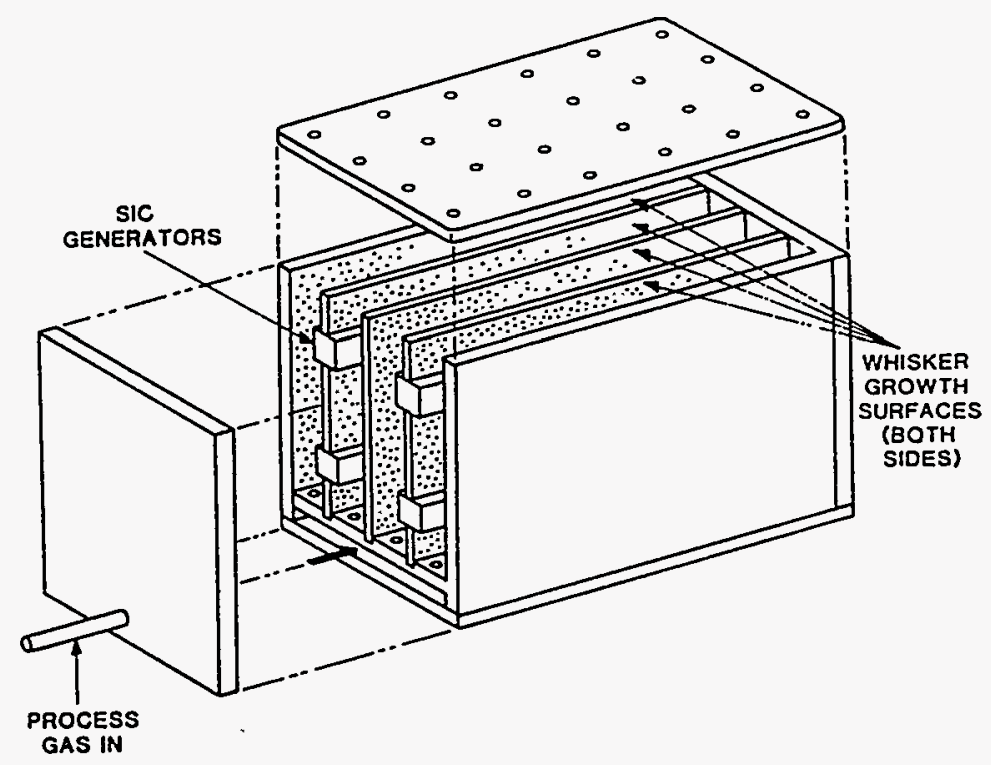

Figure 4 Los Alamos Whisker-Growing Furnace 

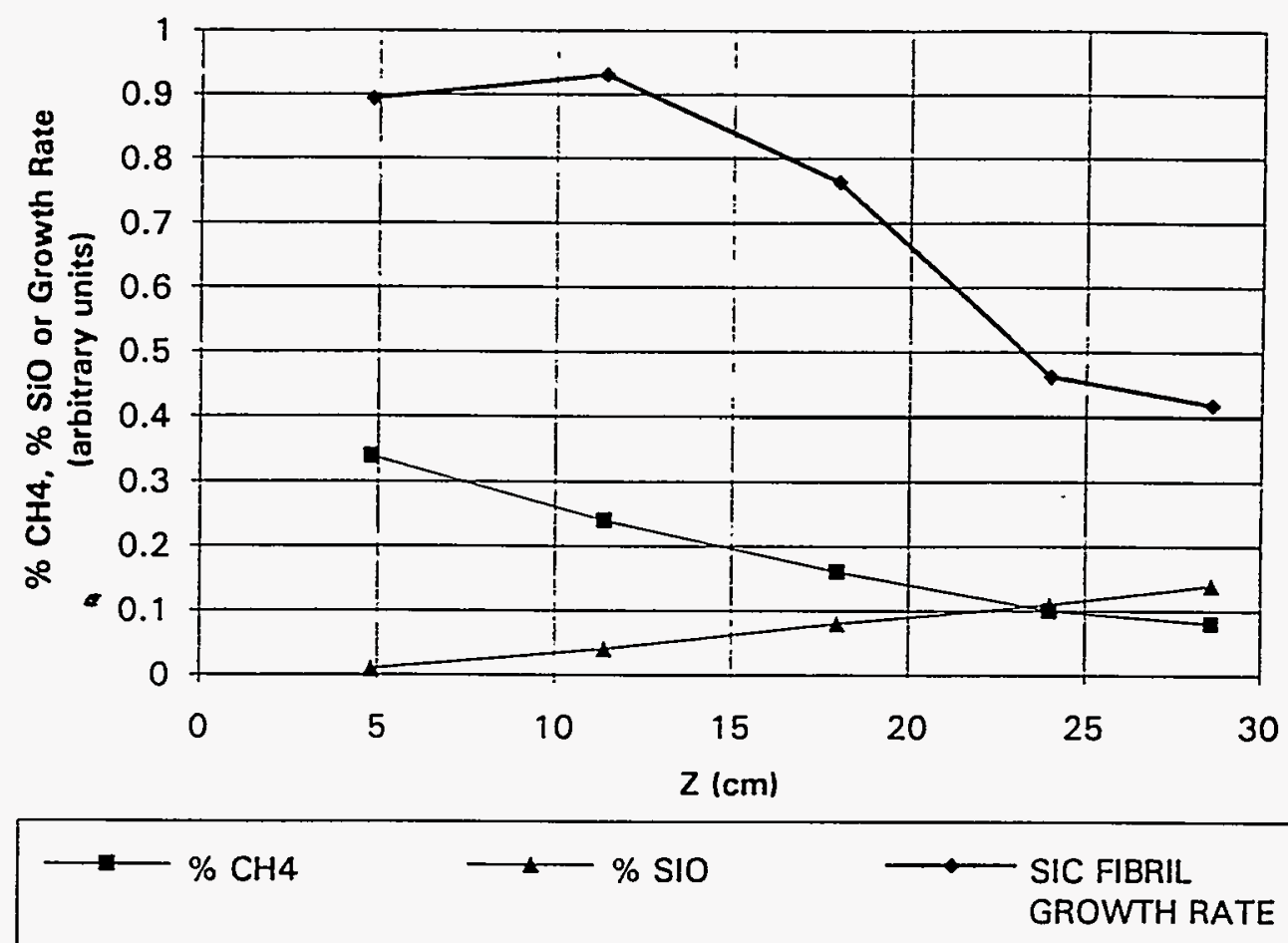

Figure 5 Effect of Reactor Position on Reactant Partial Pressures and SiC Fibril Formation Rate

Gen. 1 Gen. 2a Gen. 2b Gen. 2c
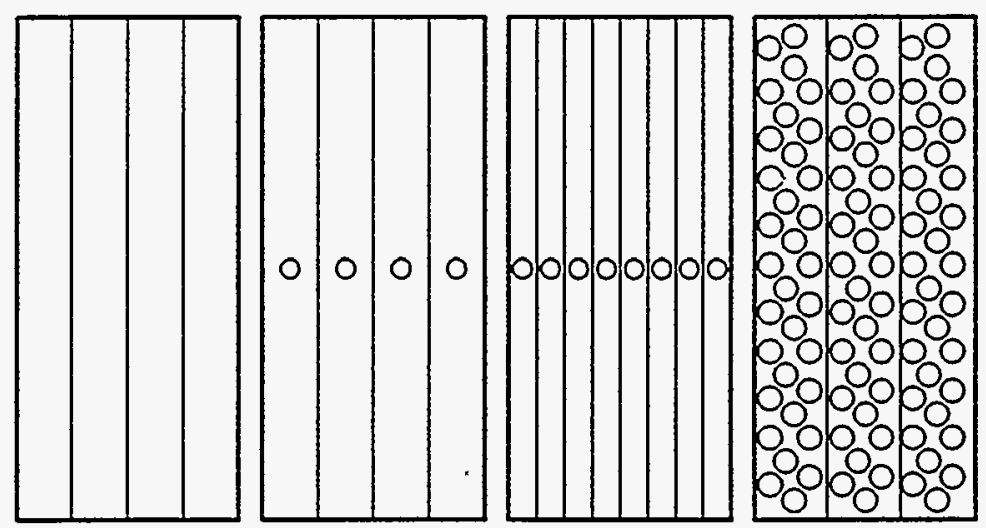

$L=$ Fibril Length $(\mathrm{cm}) \quad 1.50$

1.50

0.75

0.20

Figure 6 Reactor Design Evolution 


\subsubsection{Economic Analysis Procedure}

Two SiC fibril cost projections were conducted. The first was a conservative approach based on scaling the current process solely on economies of scale. The inputs used included furnace run time, raw-material usage, and specific growth rate. Specific growth rate values of 0.625 and $0.750 \mathrm{~g} / \mathrm{ft}^{2}-\mathrm{h}$ were used for these estimates. Although present values are $\sim 0.5 \mathrm{~g} / \mathrm{ft}^{2}-\mathrm{h}$ for the current reactor (Generation 1 in Fig. 6), the improved reactor design with additional gas inputs and closer substrate spacing (Generation $2 \mathrm{~b}$ in Fig. 6) as well as laboratory data suggest the increased values are viable estimates.

The second projection was based on a more aggressive approach that took into account the experimental data which showed a significant increase in product yield for shorter $(0.20 \mathrm{~cm})$ fibrils. Also, a shorter cycle time is used. In growing long $(>0.75 \mathrm{~cm})$ fibrils, only a small fraction of the original nucleation sites grow to maturity. The reactants are consumed by the longer growing fibrils while the shortest fibrils are deprived of reactants thereby stunting growth. By both improving process gas dispersion and decreasing the distance between growth substrates (increasing surface area), a much larger number of shorter fibrils can be grown and specific growth rates $>1.4 \mathrm{~g} / \mathrm{ft}^{2-} \mathrm{h}$ can be achieved. Additional improvement in process efficiencies can be attained by heating and cooling the furnaces more rapidly to achieve two cycles per day.

Two plant sizes were considered, one called a "prototype" plant for a production capacity of $500 \mathrm{lbs} /$ year, the other a "semiworks" plant for a production capacity of $10,000 \mathrm{lbs} /$ year. The prototype scaleup would employ a $12^{\prime \prime} \times 12^{\prime \prime} \times 42^{\prime \prime}$ reactor $(14 \mathrm{x}$ current), while the semiworks scaleup would employ a $18^{\prime \prime} \times 18^{\prime \prime} \times 56^{\prime \prime}$ reactor ( $42 \times$ current). All raw materials for SiO generation, growth substrate nucleation sites, beneficiation, and acid leaching were scaled using the same ratio as present-by volume for $\mathrm{SiO}$ generation, by surface area for $\mathrm{SiC}$ fibril nucleation, and by product yield for beneficiation and acid leaching. It has also been assumed that the process gases used $80 \%$ recycled gas for the prototype (current value) and $90 \%$ for the semiworks.

The prototype reactor $3 \mathrm{~b}$ with 12 " $\times 12$ " growth substrates was scaled from $2 \mathrm{~b}$ in Fig. 6 while $3 \mathrm{c}$ with $0.5^{\prime \prime}$ diameter $\times 12^{\prime \prime}$ long rods as growth substrates was scaled from $2 \mathrm{c}$. The semiworks reactor $4 \mathrm{~b}$ with $18^{\prime \prime} \times 18$ " growth substrates and $4 \mathrm{c}$ with $0.5^{\prime \prime}$ diameter $\times 18$ " long rods were also scaled from their $2 b$ and $2 c$ counterparts. It was assumed that these reactors would be operated horizontally with the process gas inlets located at the bottom and sides or top. In order to evaluate the effect of fibril specific growth rates, sensitivity studies with different growth rates were conducted on reactor designs $3 b$ and $4 b$ using the conservative approach.

\subsubsection{Economic Analysis Results}

Detailed process specification summaries for both conservative and aggressive approaches are given in their respective Appendices. Table 1 shows the number of furnace units 
required for the different scenarios which ranges from 2 for the aggressive prototype to 42 for the conservative semiworks.

Table 1 Number of Furnace Units Required

\begin{tabular}{|l|c|}
\hline Description & \# Furnace Units \\
\hline Conservative & \\
\hline Prototype & 6 \\
\hline Semiworks & 42 \\
\hline Aggressive & \\
\hline Prototype & 2 \\
\hline Semiworks & 12 \\
\hline
\end{tabular}

The unit production cost summaries-totals in $\$ / \mathrm{b}$ and the breakdown as a percentage of the total-are shown in Tables 2 and 3. Maintenance includes equipment repairs (5\% of installed equipment cost), supplies ( $2 \%$ of installed equipment cost), and safety devices (alarms, sensors, and shutdown switches). Indirect charges include occupancy, gas-tank rental, quality control, and depreciation.

The cost per pound of fibril product ranges from $\$ 3,800$ to $\$ 1,300$ for the conservative approach and $\$ 2,200$ to $\$ 550$ for the more aggressive approach (Table 2). All of these cost estimates are based on a 0.85 operating factor (proportion of time furnace is actually run) and a quality yield factor of 0.55 (the weight of usable fibrils based on the total grown). The 0.55 factor is very conservative since previous development work indicated that a value of $\sim 0.75$ was readily achievable. By increasing the operating factor to 0.90 and the quality yield factor to 0.85 , the $\$ / \mathrm{lb}$ can be reduced to $<\$ 800$ and $\$ 340$ for conservative and aggressive approaches respectively (Table 3). For comparison purposes a production cost sensitivity is shown in Fig. 7.

Both the conservative and aggressive approaches assume scaleup using multiple batch furnaces which are sized within the window of known furnace technology. Alternative approaches which have yet to be demonstrated but are expected to be feasible, should reduce capital, labor, and maintenance costs thereby further reducing the manufacturing costs projected here. 


\section{Table 2 Fibril Production Cost Summary (I) \\ ( 0.85 Operating Factor and 0.55 Quality Yield Factor)}

Fbri7-1

\begin{tabular}{|c|c|c|c|c|c|c|}
\hline & \multicolumn{4}{|c|}{ Conservative Approach } & \multicolumn{2}{|c|}{ Aggressive Approach } \\
\hline & \multicolumn{2}{|c|}{ Prototype } & \multicolumn{2}{|c|}{ Semiworks } & \multirow{2}{*}{$\frac{\text { Prototype }}{3 c}$} & \multirow{2}{*}{ Semiworks } \\
\hline & $3 b$ & $3 b-1$ & $4 b$ & $4 b-1$ & & \\
\hline Reactor Size, in & $12 \times 12 \times 42$ & $12 \times 12 \times 42$ & $18 \times 18 \times 56$ & $18 \times 18 \times 56$ & $12 \times 12 \times 42$ & $18 \times 18 \times 56$ \\
\hline Growth Substrate Geometry & $12^{\prime \prime} \times 12^{\prime \prime}$ & $12^{\prime \prime} \times 12^{\prime \prime}$ & $18^{\prime \prime} \times 18^{\prime \prime}$ & $18^{\prime \prime} \times 18^{\prime \prime}$ & $0.5^{\prime \prime} \mathrm{dia} 12^{\prime \prime}$ & $0.5^{\prime \prime}$ diax $18^{\prime \prime}$ \\
\hline & Plates & Plates & Plates & Plates & Rods & Rods \\
\hline Typical Fibril Length, cm & 0.75 & 0.75 & 0.75 & 0.75 & 0.20 & 0.20 \\
\hline Specific Growth Rate, g/ft2-h & 0.625 & 0.750 & 0.625 & 0.750 & 1.429 & 1.429 \\
\hline Hours/Cycle & 14.5 & 14.5 & 14.5 & 14.5 & 12.0 & 12.0 \\
\hline Cycles/Unit/Year & 436 & 363 & 446 & 371 & 423 & 512 \\
\hline Days/Year Used & 310 & 258 & 317 & 264 & 249 & 301 \\
\hline Pounds Fibrils/Year & 500 & 499 & 9991 & 9984 & 500 & 10014 \\
\hline No. Furnace Units & 6 & 6 & 42 & 42 & 2 & 12 \\
\hline No. Professional Employees & 1 & 1 & 4 & 4 & 1 & 3 \\
\hline No. Technical Employees & 6 & 6 & 30 & 30 & 4 & 12 \\
\hline Unit Production Cost, $\$ / \mathrm{lb}$ & & & & & & \\
\hline Raw Materials & 80 & 72 & 82 & 74 & 45 & 46 \\
\hline Utilities & & & & & & \\
\hline ElectricityMater & 8 & 7 & 6 & 5 & 3 & 2 \\
\hline Process Gases & 570 & 476 & 306 & 254 & 184 & 100 \\
\hline Labor & 1665 & 1387 & 399 & 332 & 1225 & 184 \\
\hline Maintenance & 605 & 606 & 261 & 261 & 257 & 83 \\
\hline Indirect Charges & 874 & 875 & 382 & 381 & 480 & 134 \\
\hline Total Unit Production Cost, $\$ / \mathrm{lb}$ & 3802 & 3423 & 1436 & 1306 & 2194 & 549 \\
\hline
\end{tabular}




\section{Table 3 Fibril Production Cost Summary (II)}

(0.90 Operating Factor and 0.85 Quality Yield Factor)

\begin{tabular}{|c|c|c|c|c|c|c|}
\hline & \multicolumn{4}{|c|}{ Conservative Approach } & \multicolumn{2}{|c|}{ Aggressive Approach } \\
\hline & \multicolumn{2}{|c|}{ Prototype } & \multicolumn{2}{|c|}{ Semiworks } & \multirow{2}{*}{$\frac{\text { Prototype }}{3 c}$} & \multirow{2}{*}{$\begin{array}{c}\text { Semiworks } \\
4 c\end{array}$} \\
\hline & $3 b$ & $3 b-1$ & $4 b$ & $4 b-1$ & & \\
\hline Reactor Size, in & $12 \times 12 \times 42$ & $12 \times 12 \times 42$ & $18 \times 18 \times 56$ & $18 \times 18 \times 56$ & $12 \times 12 \times 42$ & $18 \times 18 \times 56$ \\
\hline Growth Substrate Geometry & $12^{\prime \prime} \times 12^{\prime \prime}$ & $12^{11} \times 12^{\prime \prime}$ & $18 " x 18 "$ & $18^{\prime \prime} \times 18^{\prime \prime}$ & $0.5^{\prime \prime} d i a x 12 "$ & $0.5^{\prime \prime}$ diax18" \\
\hline & Plates & Plates & Plates & Plates & Rods & Rods \\
\hline Typical Fibril Length, cm & 0.75 & 0.75 & 0.75 & 0.75 & 0.20 & 0.20 \\
\hline Specific Growth Rate, g/ft2-h & 0.625 & 0.750 & 0.625 & 0.750 & 1.429 & 1.429 \\
\hline Hours/Cycle & 14.5 & 14.5 & 14.5 & 14.5 & 12.0 & 12.0 \\
\hline Cycles/Unit/Year & 462 & 384 & 472 & 393 & 448 & 542 \\
\hline Days/Year Used & 310 & 258 & 317 & 264 & 249 & 301 \\
\hline Pounds Fibrils/Year & 818 & 817 & 16348 & 16338 & 818 & 16386 \\
\hline No. Furnace Units & 6 & 6 & 42 & 42 & 2 & 12 \\
\hline No. Professional Employees & 1 & 1 & 4 & 4 & 1 & 3 \\
\hline No. Technical Employees & 6 & 6 & 30 & 30 & 4 & 12 \\
\hline Total Unit Production Cost, $\$ / \mathrm{lb}^{\star}$ & 2323 & 2092 & 878 & 798 & 1341 & 335 \\
\hline
\end{tabular}

*Table 2 values $\times(0.85 \times 0.55) /(0.90 \times 0.85)$ 


\section{Figure 7 Operating Cost $(\$ / / \mathrm{lb})$ Sensitivities}

Conservative Approach

Operating Cost $(\$ / \mathrm{lb})$

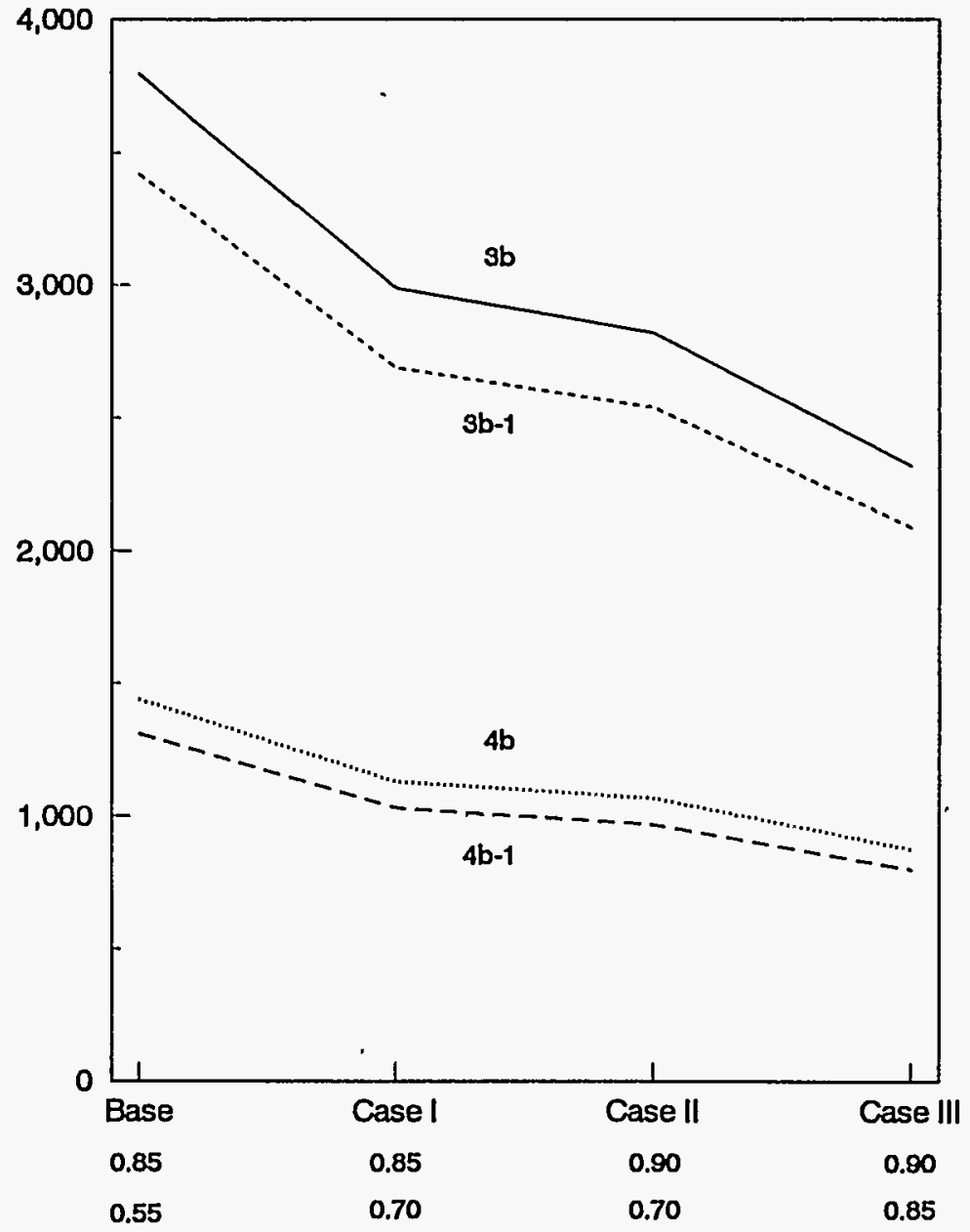

Aggressive Approach

Operating Cost (\$/lb)

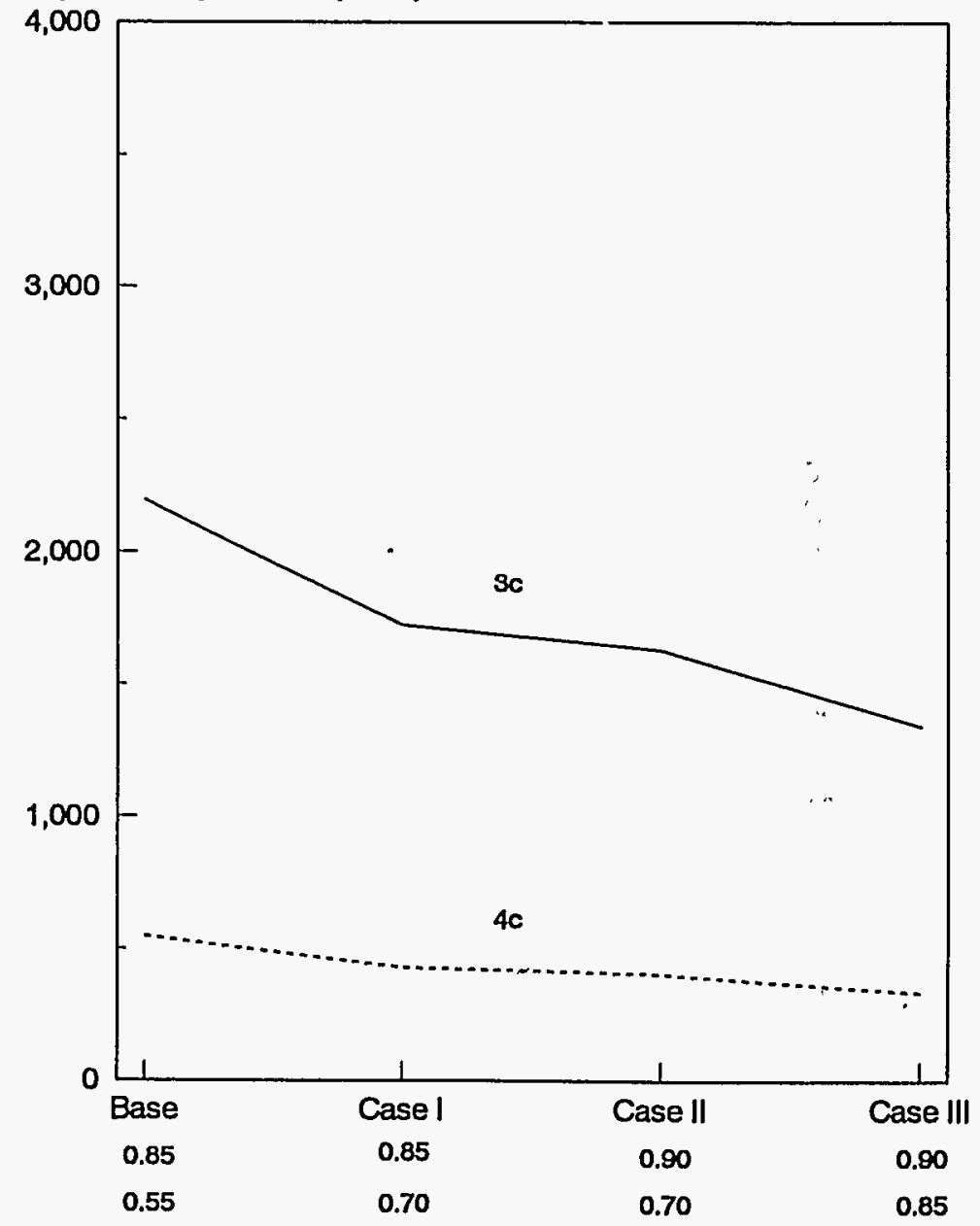




\section{Acknowledgments}

The authors gratefully acknowledge the following individuals who made the successful completion of this program possible:

Carborundum coworkers:

George Glijer and Mike Heath for their efforts in performing and analyzing the fibril-growth experiments;

Steve Lacki for SEM analysis of SiC fibrils;

Joe Biernacki and Mike Tenhover for insightful technical discussions;

Bonnie Mehls and Debra Hasley for contractual, accounting, and administrative support.

Frank Ko and Kathleen Schatz of Advanced Product Development for their cooperation and painstaking effort in yarn fabrication;

Nancy Cole and Ernie Long of DOE/ORNL for their support and encouragement throughout this program. 


\section{References}

1. Shalek, P.D., D.E. Christiansen, F.D. Gac, R.E. Honnell, G.F. Hurley, J.D. Katz, W.J. Parkinson, J.J. Petrovic, and D.S. Phillips, "Scale-Up and Optimization of the VLS Growth Process for Beta-SiC Whiskers for the Period April 1982 September 1989," DARPA Final Report \& DOE/OAR\&TD Topical Report, Los Alamos National Laboratory Report \#LA-12119 (1992).

2. Hollar, Jr., W.E. and W.H. Mills, "Engineering Scale Development of the VaporLiquid-Solid (VLS) Process for the Production of Silicon Carbide Fibrils," ORNL/Sub/86-SD861/01, September 1993.

3. Gac, F.D., "Synthesis and Characterization of VLS Silicon Carbide Whisker Reinforced Reaction Bonded Silicon Nitride," Ph.D. Thesis, University of Washington (1989).

4. Christiansen, D.E. and B. Van Eeckhout, Chem. Eng. Prog., 84, 18-22 (1988).

5. Hollar, W.E. and J.J. Kim, "Review of VLS Whisker Growth Technology," Ceram. Eng. \& Sci. Proc., 12 [7-8], 979-991 (1991). 



\section{Appendix I}

Conversion of SiC Fibrils to Linear, Aligned Fibrous Assemblies for Ceramic Matrix Composites 


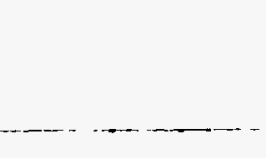




\title{
CONVERSION OF SiC FIBRILS \\ TO LINEAR , ALIGNED FIBROUS ASSEMBLIES \\ FOR CERAMIC MATRIX COMPOSITES
}

\author{
A Finai Report Submitted to \\ The Carborundum Company \\ Wendel Tech Center \\ Acheson Drive \\ Niagara Falls, NY 14303 \\ Attn.: Bonnie L. Mehls \\ Tel: (716) 278-2585 \\ Fax: (716) 278-2225
}

DOE Fossil Energy Materials AR \&TD Materials Program

Contract No: 86X-SD861C

Program Manager: Nancy Cole

Program Monitor: Ernie Long

By

Kathleen Schatz - Project Engineer

Frank K. Ko - Project Manager

George Du - Project Scientist

Rick Balonis - Process Engineer

Frank Scardino - Project consultant

September 5, 1996

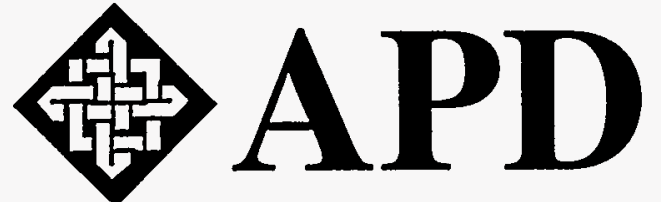

Advanced Product Development, 2500 Pearl Buck Rd, Bristol, PA 19007, USA

Phone (215) 785-3230 Fax (215) 785-3123 



\section{TABLE OF CONTENTS}

Page

EXECUTIVE SUMMARY

1. INTRODUCTION 4

1.1 Comparison of SiC Fibril with Textile Fibers 6

1.2 Four Basic Steps in Yarn Manufacturing 7

1.3 Nontraditional Fiber Processing 9

1.3.1 Adhesive-Tape Method 9

1.3.2 Wet-Laying Method 10

1.4 Experimental Procedure 11

2. TRADITIONAL TEXTILE PROCESSING METHODS 13

2.1 Model Fiber - Nicalon SiC 13

2.2 Experimental

- Textile Machinery Requirements for Yarn Manufacturing

14

- Textile Machinery Modifications 16

2.2.1 Hand-Carding Experiments 18

- Alternative Card Clothing 22

- Carding Process Conclusion 23

2.2.2 Air-Vortex Spinning 23

- Spinning Process Conclusion 25

$\begin{array}{lll}2.3 & \text { Summary } & 25\end{array}$

3. NONTRADITIONAL TEXTILE PROCESSING METHODS 25

3.1 Adhesive-Tape Process 26

3.1.1 Introduction 26

- Initial Adhesive-Tape Concept Model 26

- Fibril Plate Scheduling 27

- Fibril Plate Growth Variations 27

3.1.2 Separation and Alignment 28

3.1.3 Catalyst Ball Removal and Adhesive-Tape Integration 31

3.1.4 Adhesive-Tape Application and Handling 32 


\section{TABLE OF CONTENTS}

(Continued)

3.1.5 Linear Density Of Fibril Tapes 34

3.1.6 Summary $\quad 35$

3.2 Fluid-Based Processing 35

3.2.1 Introduction $\quad 35$

3.2.2 Dispersion 38

3.2.3 Orientation and Accumulation 41

3.2.4 Integration $\quad 45$

3.2.5 Characterization of Linear Fibril Assemblies 46

- Textile Evaluation 46

- Tensile Testing 47

- Flexural Testing 48

- Composite Reinforcement Evaluation 49

- Fibril Orientation 50

- Fibril Volume Fraction $\quad 50$

4. CONCLUSIONS AND RECOMMENDATIONS 51

- Flow Studies $\quad 53$

- Dispersion 53

- Subsequent Processing 54

- Integration $\quad 54$

- Textile Preforming 54

- Machine Nesign $\quad 55$

5. REFERENCES $\quad 57$

$\begin{array}{ll}\text { ACKNOWLEDGMENTS } & 60\end{array}$

6. APPENDICES

1. Alternatives to Traditional Textile Methods A-1

2. Comparison of SiC Fibrils \& Textile Processed Asbestos A-3

3. Comparison of SiC Fibrils \& Chopped Nicalon Fiber A-4

4. Fillet Clothing Carding Experimental Procedure A-5

5. Alternatives to Fillet Card Clothing A-8

6. Areal Density of Harvested Fibrils A-10

7. Photographs from Fibril Orienting Experiments A-11

8. Clean Room and Safety Procedures A-14 


\section{Executive Summary}

Preform fiber architecture has been demonstrated to play an important role in facilitating processing and enhancing the toughness of ceramic matrix composites. ${ }^{1}$ In order to fully exploit the superior strength, modulus, and thermal stability of the SiC fibril such as the one currently produced by Carborundum, considerable effort has been devoted in the industry to convert the fibrils to linear fibrous assemblies. ${ }^{2}{ }^{3}$ As illustrated in Figure 1 (which plots critical fiber diameter as a function of tensile modulus), the SiC fibril (with a modulus of approximately $600 \mathrm{GPa}$ and a diameter of $5 \pm 3 \mu \mathrm{m}$ ) is on the fringe of being processable as a textile fiber because its fine diameter can compensate for its high modulus.

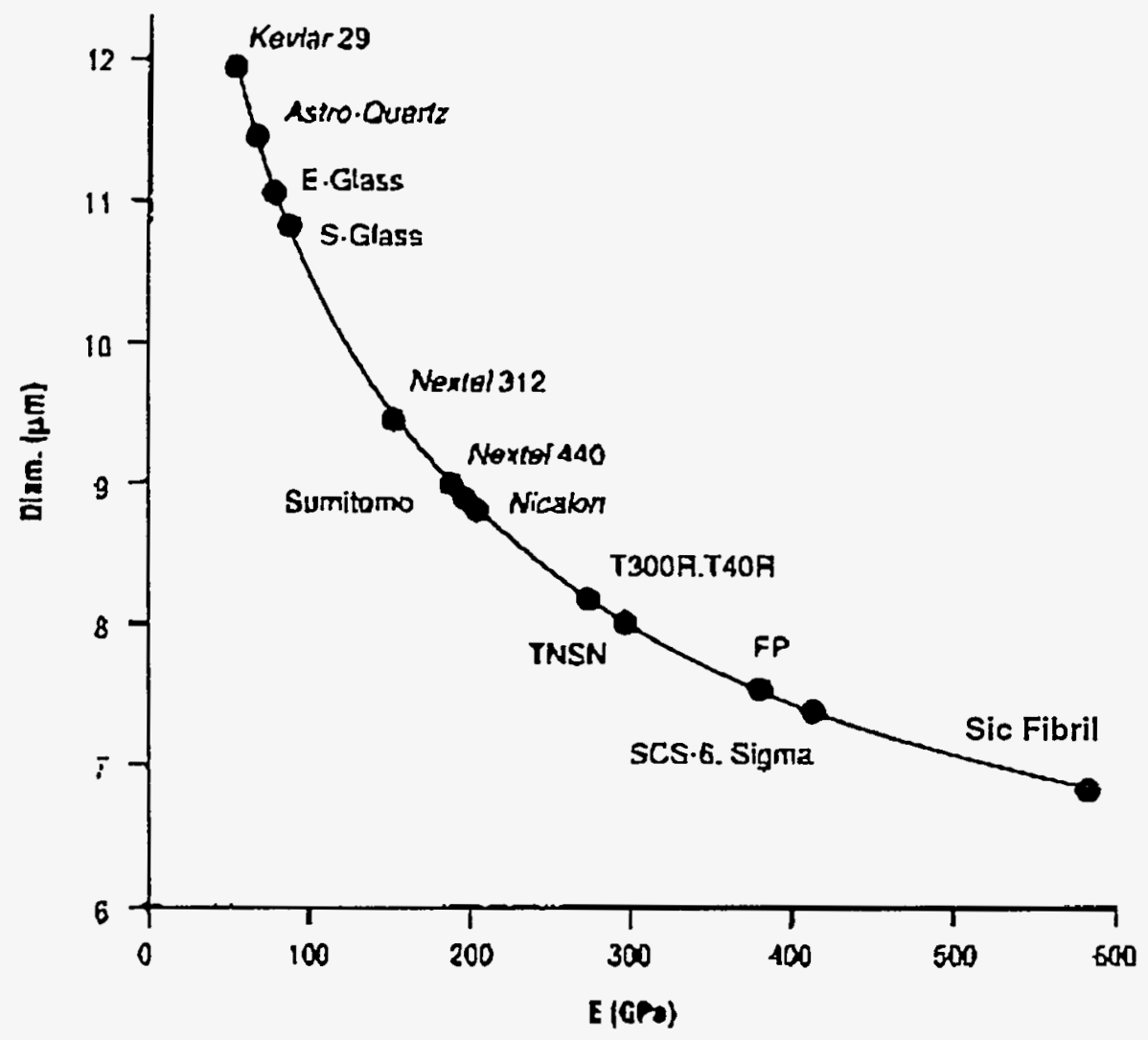

Figure 1. Critical fiber diameter for successful textile processing. ${ }^{4}$

IF. Ko, "Preform Fiber Architecture for Ceramic Matrix Composites," Bulletin of American Ceramic Society, Vol. 68, No. 2, 1989.

${ }^{2}$ NASA Contractor Report CR-785, May 1967.

${ }^{3}$ W. Sutton, Principles and Methods for Fabricating Whisker Reinforced Composites.

${ }^{4}$ F. Ko, and C. Pastore, and A.Head, Handhook of Industrial Braiding. Covington, KY: Atkins \& Pearce, 1990, p. 2-9. 
In order to assess the feasibility of converting the $\mathrm{SiC}$ fibril to fibrous assemblies, Phase II of the program was designed to examine the key technical issues in the processing of $\mathrm{SiC}$ fibrils. It $\mathrm{was}$ identified that, similar to traditional textile yarn processing, dispersion, alignment, and cohesion of the fibril assemblies are the essential ingredients for the successful conversion of the fibril to a textile-like linear fibrous assembly.

During the Phase II of this program, the fibrils were subjected to modified lab-scale textile processes including carding and airvortex spinning. These processes failed to produce a cohesive fiber assembly due to the brittleness of the fibril and surface damage resulting from mechanical surface contact. In an effort to contain the fibrils (minimize the exposure), enhance the structural integrity, and simplify the conversion process (directly from harvesting to yarn), the feasibility of a sandwiched tape process was evaluated. While the structural integrity issue was demonstrated in woven fibril tape samples, difficulties were encountered in the removal of catalyst balls. The most notable drawback of the tape yarn process was the failure to align the fibrils.

It was concluded that a fluid-based (wet) process will provide the most gentle media for the dispersion, aligning, and integration of the fibrils. A manual experimental batch process was established to produce linear fibrous assemblies through dispersion and subsequent alignment of the fibrils by fluid flow. The interfibril cohesion was provided both by the use of chemical binders and different auxiliary sheath techniques. As illustrated in Figure 2, a high level of fibril alignment was achieved by the wet process. These aligned fibril linear assemblies will be fabricated into ceramic composites by the Chemical Vapor Deposition (CVD) process for preliminary characterization of the properties of the ceramic matrix composites. 


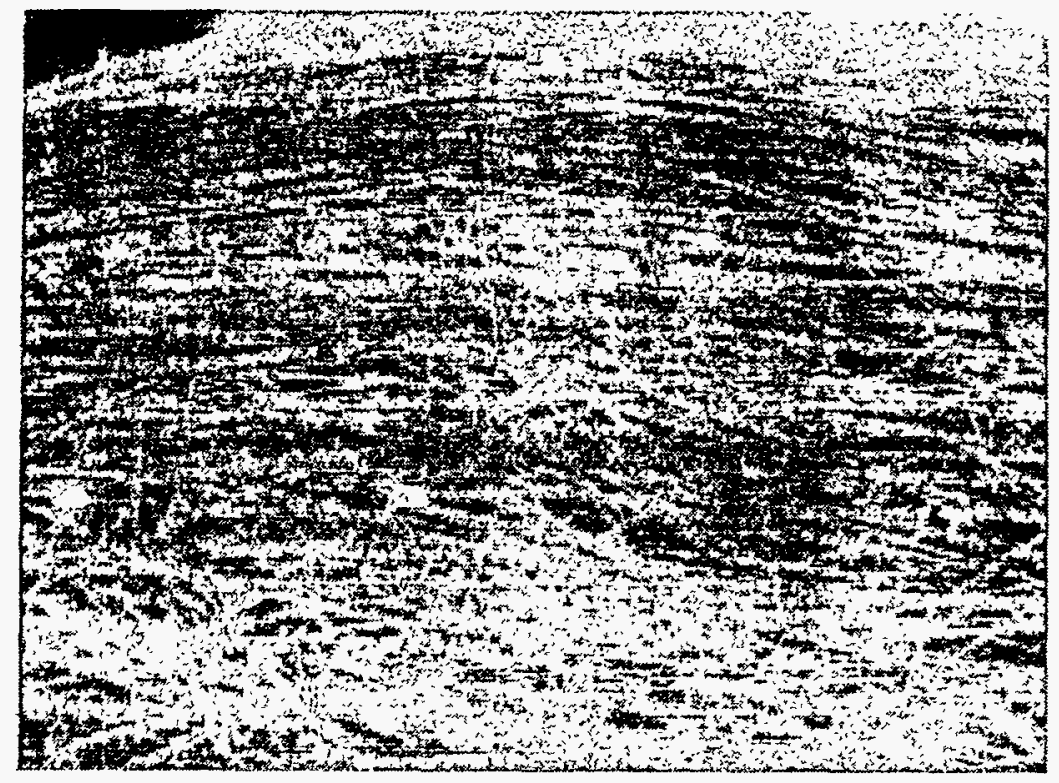

Figure 2a. Magnification of fibrils aligned by current lab-scale wetlaid processing.

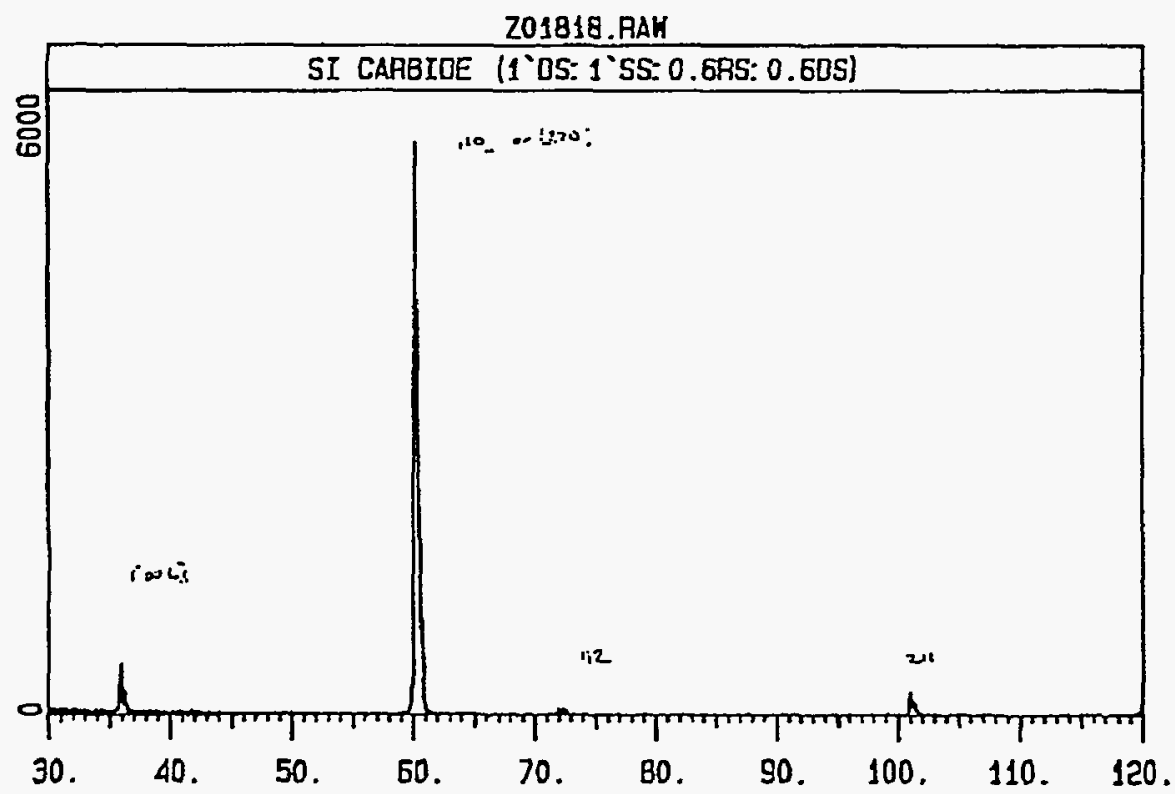

Figure $2 b$. Measurement of fibril alignment by X-ray diffractometry.

With the successful demonstration of the feasibility of the wet process in Phase II of the program, it is our objective to optimize the processing parameters and establish a lab-scale semicontinuous process to produce the wet-laid fibril yarns through a future program with the Department of Energy. 


\section{Introduction}

During Phase II of this program, APD, Inc. explored the established lab-scale textile yarn manufacturing processes of carding and spinning. It has been concluded that these conventional textile processes are unsuitable for processing Carborundum $\mathrm{SiC}$ single crystal fibrils. Building on that work and past experience generated by Los Alamos National Laboratory, APD has completed additional work in two nontraditional textile yarn-forming methods including the adhesive-tape method and the wet-laid method. Experiments in both methods have been useful in characterizing fibril growth and processing parameters. The wet-laid method exhibits the most promise for further study because it alone fulfilled a key requirement of this program--to create a linear fibril assembly with oriented fibrils.

The course of this program has been defined primarily by the empirical application of textile engineering principles. Diverse categories of materials engineering were utilized as the work progressed beyond traditional textile processing. Simplified textile evaluation techniques and microscopy were used to characterize the linear fibril assemblies.

Figure 3 shows the hierarchical relationships between fiber, yarn, and fabric structure and properties.

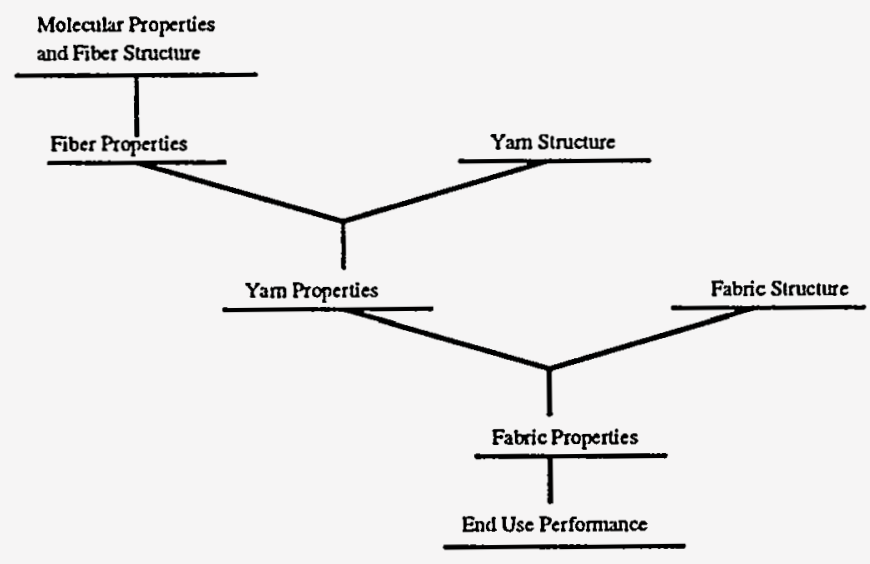

Figure 3. Hierarchical relationships between fiber, yarn, and fabric properties and structures. ${ }^{5}$

${ }^{5}$ I. W. S. Hearle, P. Grosberg, and S. Backer, The Structural Mechanics of Fibers, Yarns, and Fabrics. Wiley-Interscience, New York, 1969. 
In Figure 4 the specific fibril structure and properties are incorporated in the diagram along with the required yarn and fabric properties necessary for the end-use performance as reinforcement in ceramic-ceramic composites. The two headings left without details, namely yarn structure and fabric structure, are the specific areas in which research and development are required. Developing a specific yarn structure has been the focus of the Phase II effort.
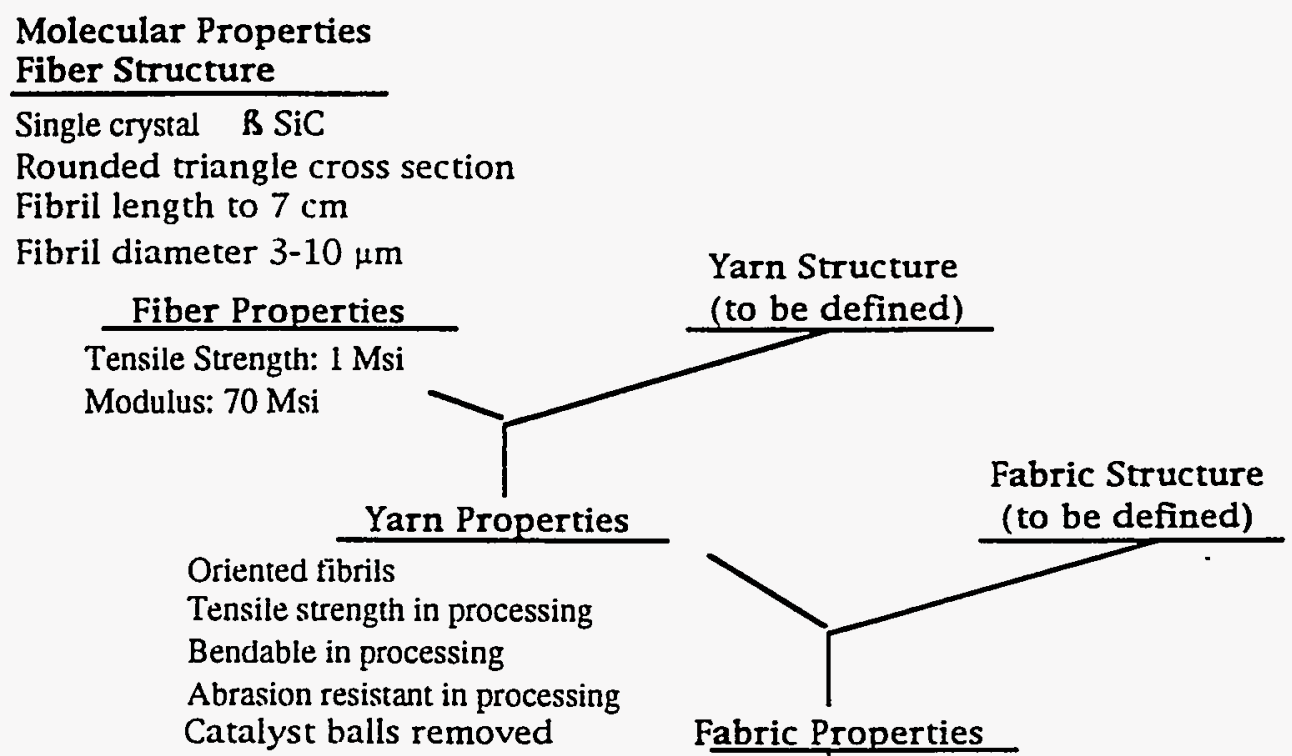

Figure 4. $\mathrm{SiC}$ fibril properties and relationships with yarn and fabric properties.

Listed under the "Yarn Properties" heading in Figure 4 are the requirements of an $\mathrm{SiC}$ fibril yarn. The first requirement, to produce oriented fibrils, is critical to the full exploitation of the unique properties of the fibril. Oriented fibrils in a yarn would allow for the design of a textile reinforcement for high temperature composites. The orientation is thus the primary criteria for a fibril yarn. Implementing a composite preform design, through 3-D braiding, weaving, etc., requires yarn properties which allow the oriented fibiils to be processed. The next two listed yarn properties are 
tensile strength and bending behavior. Tensile strength is the property which allows the yarn to be processed into fabric by being threaded through guides, onto rolls, etc., without breaking. The fabric cannot be made, however, if there is not sufficient bending ability in the yarn to allow it to conform to other yarns. An additional requirement is abrasion resistance to prevent loss of fibrils and fibril dust for economic and safety reasons.

The primary focus of this program has been the development of a method to orient the fibrils. Seeking the additional structure and process which produces the textile processing properties is the critical second step. As shown in Figure 4, the "yarn structure and fiber structure" are to be defined in the program.

Accordingly, our objective of this study is to investigate 1) the trade-offs which are inherent in developing a yarn from SiC fibrils, 2) the process rationale behind the development of the fiber, and 3 ) the results of the experiments performed during this development.

\subsection{Comparison of SiC Fibril with Textile Fibers}

Unlike other high performance composite reinforcements which are produced in filament (continuous) lengths, the single crystal fibrils are only available in short (discrete) lengths. Processing of fibrils must begin with a consideration of the methods by which short fibers have been processed in the textile industry for centuries. The distinction between the short fibers (called staple-length fibers) in the industry and the fibrils is necessary in order to understand the promise and limitations of textile processing.

The two photographs in Figure 5 show a typical short textile fiber (cotton) and the $\mathrm{SiC}$ fibrils. Comparing the photographs reveals other differences between the two fibrous materials and consequently the challenges of converting the $\mathrm{SiC}$ fibrils into yarn.

The first difference is the dimensional size. The SiC fibrils are much shorter and finer than the cotton fiber. The second difference is that the fibrils do not have crimp. Crimp is the "wavy" configuration the cotton fiber exhibits. All short textile fibers which are processed have crimp, whether occurring naturally in cotton or wool or manufactured in man-made fibers such as polyester, rayon, etc. Another very important difference between the fibers and 
fibrils are the intricate properties--the fibrils having extremely high tensile and modulus values. These values make the fibrils behave more like rigid rods than flexible fibers. A final difference is cost. Cotton sells for approximately $\$ 1-\$ 2$ per pound and the fibrils currently sell for over $\$ 200,000$ per pound, ${ }^{6}$ because of the limited availability of fibrils.

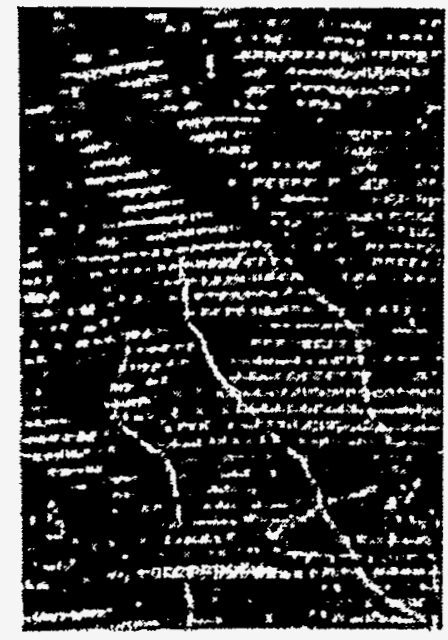

a

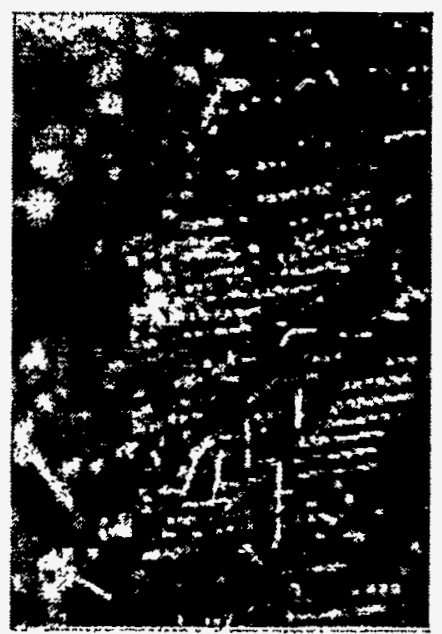

b

Figure 5. Showing a) cotton fiber and b) $\mathrm{SiC}$ fibrils (shown on fabric background)

\subsection{Four Basic Steps in Yarn Manufacturing}

Traditional textile processing of short fiber into yarn consists of four basic steps: separation, alignment, attenuation, and integration. (In reading the following sections it is important to understand that variations of these basic steps are three processing approaches tested by APD.)

In traditional textile conversion of staple-length fibers to yarn, the process begins with bales of compressed, entangled fiber from either natural sources (cotton or wool) or man-made sources (staplelength rayon, polyester, etc.). The multiple, progressive processes of the spinning operation serve to reduce and organize the masses of fiber into a strong, linear fiber assembly or yarn.

${ }^{6}$ Price quote. Carborundum to Advanced Product Development, Inc., January 1995. 
The process begins by releasing the bales and feeding the fiber through a series of machines to pull apart the masses of fiber into loose tufts. The fibers separate from the tufts when fed through a machine called a card. It is this process that converts a mass of fibers into a semistructured form. Fiber from the opening stage is fed into the machine and brought between two sets of points. The actual carding is done by these points which are present on a large cylinder and on point-covered flats that rotate over the top portion of the cylinder. These points (called the card clothing) do the work of separating and pulling the fibers into a thin web. Through this action the fibers are separated and aligned. The resulting web is transferred off the cylinder and condensed into a rope-like strand called sliver. The sliver passes through other machinery to attenuate (reduce in size), which further promotes the alignment of the fibers. When the desired linear weight is reached, the now-reduced strand

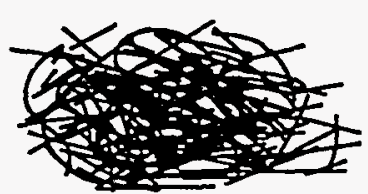

1. Mass of entangled fiber.

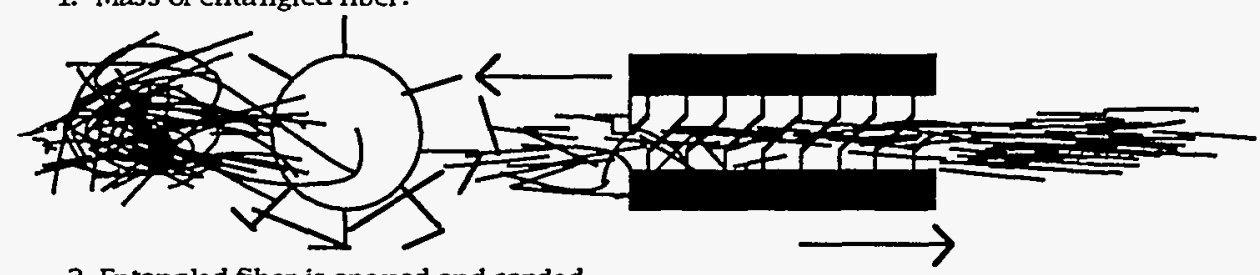

2. Entangled fiber is opened and carded.

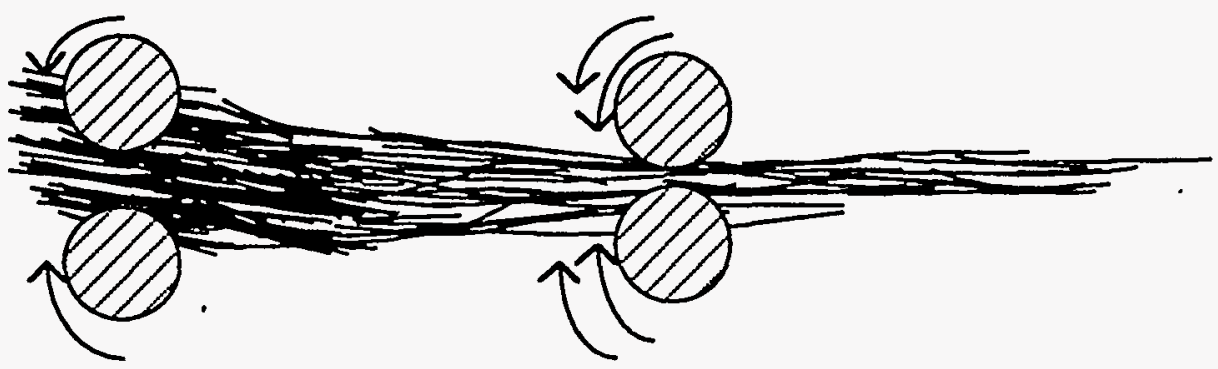

3. Sliver is a ttentuated to roving.

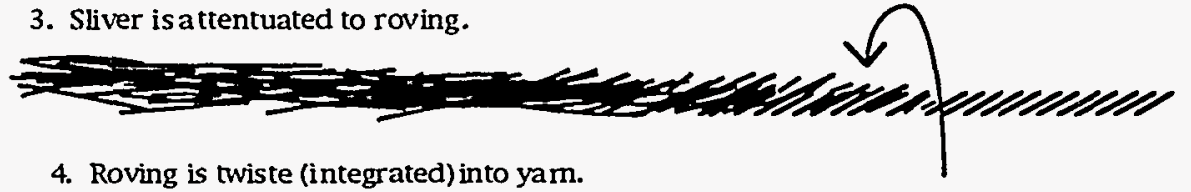

Figure 6. Schematic of traditional textile processing.

(roving) is twisted to integrate the fibers and promote tensile strength through frictional forces between the fibers (Figure 6). 


\subsection{Nontraditional Fiber Processing}

The nontraditional fiber processing employed in this program includes the adhesive-tape method and the wet-laying method. These processes, which form basic steps of textile yarn processing, are broadly interpreted and adapted accordingly.

\subsubsection{Adhesive-Tape Method}

In the adhesive-tape method, separation and alignment occur while the fibrils were still anchored on the growth plate, through a combing or brushing step. Like hair on a scalp, fibril entanglement is limited to the entanglement which is formed as the fibrils grow together and can be modified by controlling growth density and fibril growth length. Once oriented, the fibrils can be removed from the plate by applying and removing an adhesive tape, which provides the integration. In this process, an attenuation step does not occur, as the linear weight is dictated by the fibril growth on the plate (Figure 7).

\section{ADHESIVE -TAPE METHOD}

1. Reorient fibrils on plate by combing/brushing so that fibrils are as parallel to plate and to each other as possible.

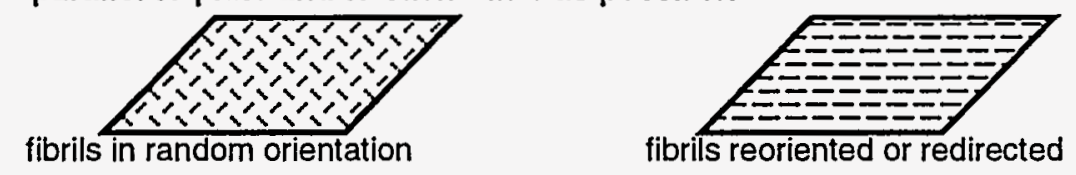

2. Transfer fibrils from plate to adhesive tape in a continuous manner.

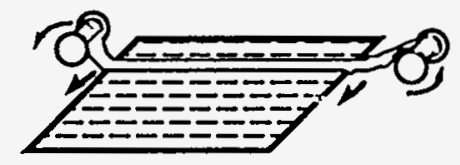

3. Laminate with a second tape layer.

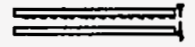

다를

4. Cut or slit laminate to desired width for a laminated fibril yarn.

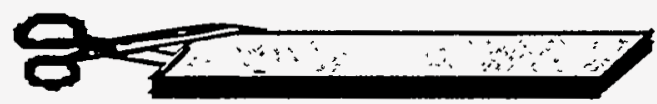

Figure 7. Schematic of the adhesive-tape method. 


\subsubsection{Wet-Laying Method}

In the wet-laying method, the first step is the dispersion of fibrils in a liquid medium to the degree that the fibrils are discretely suspended. This corresponds to the opening and separation steps of traditional spinning. Alignment occurs when the liquid medium is allowed to flow in a controlled manner and the dispersed fibrils align in the flow onto a porous screen where the liquid is removed. Integration occurs with a binder and the addition of a substrate which serves to give the assembly strength. Attenuation and accumulation of fibrils to increase the linear veight are possible (Figure 8 ) by varying the take-up and deposition speed.
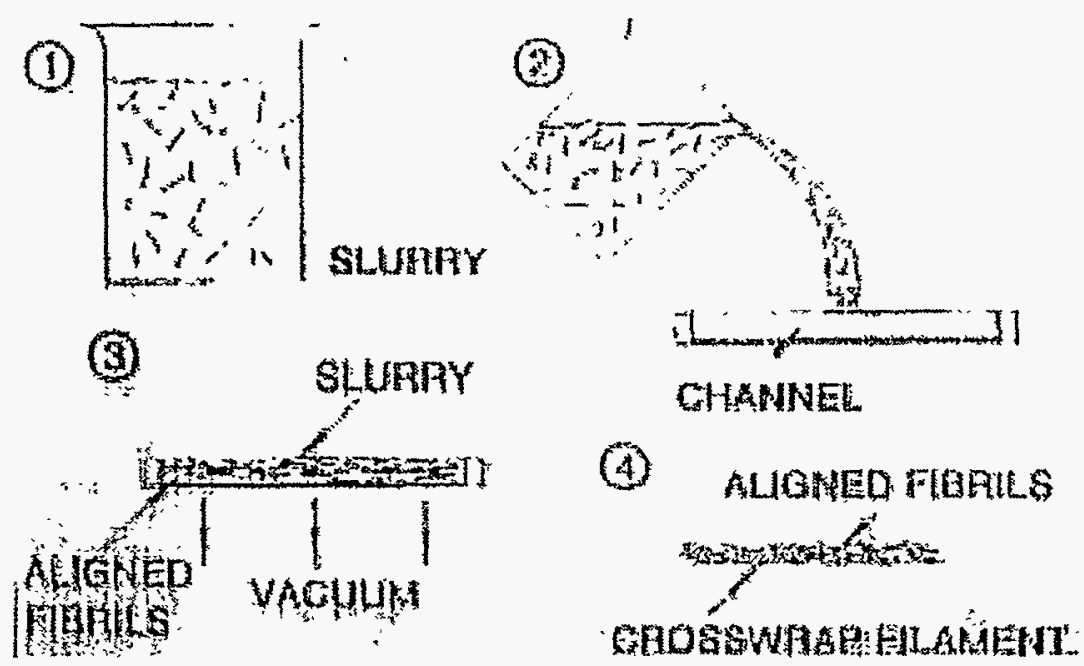

Figure 8. Schematic of the fluid-based, wet-laid system.

Table 1 summarizes how the four basic processing steps occur in traditional and nontraditional fiber processing methods.

Of the three processing methods tested, the most promising is the wet-laid system, which has proven most successful in providing a way to separate and align the fibrils.

In the last stage of the program, efforts concentrated on the demonstration of the feasibility of the wet-laying process relative to its ability to align the fibrils. Additional questions that remain to be answered are the robustness of the process and the feasibility to scaleup to a carbon fiber. 
The following section provides chronological descriptions of the development of the linear fibril assembles.

\section{Table 1. Basic Processing Steps in the Traditional and Nontraditional Textle Processing Methods}

\begin{tabular}{|l|l|l|l|}
\hline & $\begin{array}{l}\text { Traditional } \\
\text { Textile } \\
\text { Processing }\end{array}$ & $\begin{array}{l}\text { Adhesive-Tape } \\
\text { Processing }\end{array}$ & $\begin{array}{l}\text { Wet-Laid } \\
\text { Processing }\end{array}$ \\
\hline 1) Sefaration & Opening, Carding & $\begin{array}{l}\text { Aligning on } \\
\text { growth plate }\end{array}$ & $\begin{array}{l}\text { Dispersion in } \\
\text { liquid medium }\end{array}$ \\
\hline 2) Alignment & Carding & $\begin{array}{l}\text { Aligning on } \\
\text { growth plate }\end{array}$ & $\begin{array}{l}\text { Alignment in } \\
\text { flow of } \\
\text { dispersion } \\
\text { medium }\end{array}$ \\
\hline $\begin{array}{l}\text { 3) Attenuation } \\
\text { or other control } \\
\text { of linear } \\
\text { density of } \\
\text { fibrils }\end{array}$ & Drafting & $\begin{array}{l}\text { None; } \\
\text { dependent on } \\
\text { fibril growth } \\
\text { density and } \\
\text { length }\end{array}$ & Accumulation \\
\hline $\begin{array}{l}\text { 4) Integration } \\
\text { Spinning }\end{array}$ & $\begin{array}{l}\text { Adhesive-tape } \\
\text { application }\end{array}$ & $\begin{array}{l}\text { Binder and } \\
\text { auxiliary tape } \\
\text { substrate }\end{array}$ \\
\hline
\end{tabular}

\subsection{Experimental Procedure}

The program began with the assumption that the production of SiC fibril yarns can be modeled after the industrial success of the textile processing of asbestos fibers. This is based on the assumption of the similarities between asbestos fibers and the $\mathrm{SiC}$ fibrils which include a crystalline fiber growth character, fiber fineness, and crimpless characteristic (Appendix 1). It was believed that the $\mathrm{SiC}$ fibrils, similar to asbestos fibers, could be blended with traditional textile fibers and processed into yarns on modified textile machinery. An advantage was seen in the fibril growth imperfections of bambooing and branching.? These imperfections and the fibril growth catalyst balls were viewed as potentially useful as a crimp substitute providing interfibril cohesion.

${ }^{7}$ The Carborundum Company, Report Issued September 1993, "Engineering Scale Development of the Vapor-Liquid-Solid (VLS) Process for the Production of Silicon Carbide Fibrils", Figures 21 and 22. 
The requisite textile machinery was procured and modifications were made. Bulk, chopped Nicalon was chosen as a modeling fiber for its similarities to the $\mathrm{SiC}$ fibrils (high modulus, crimpless form) and traditional spinning fibers were used as blending fibers.

The results of processing the Nicalon fiber alone and in blends on the textile machinery were unacceptable. Carding could not adequately separate the fibers, resulting in minimum blending with other fibers. No discernible orientation was observed. Breakage of the Nicalon fiber was observed. The uneven blending led to inconsistent roving which could not be processed in the subsequent operations of drawing and air-vortex spinning. An acceptable sample of a Nicalon blended yarn was not produced by the traditional textile process.

The inability to process the Nicalon was attributed to the high fiber modulus and crimpless nature. It was concluded that greater difficulties will be encountered in the fibril which has more than twice the modulus of Nicalon fiber. Alternatives to traditional textile processing were required which would still accomplish the four processing steps of separation, alignment, attenuation, and integration. The two alternative methods targeted for work were the adhesive-tape method and the wet-laid method.

The adhesive-tape and wet-laid methods were based on ideas meant to simplify and enhance the results of the four processing steps.

The adhesive-tape method was based on simplifying the fibril separation and alignment steps. Instead of working with harvested, entangled fibrils in the traditional carding operation, it was believed that the fibrils, while still attached on the growth plate, could be more readily separated and oriented, particularly because the fibril growth length and density could be controlled. Once oriented, adhesive tape would be applied to the oriented fibrils for integration purposes and the tape and fibril assembly would then be removed from the plate. Minimum handling and breakage of the fibrils were thought to be the prime advantages to this method.

The wet-laid method was based on the work attempted at Los Alamos National Laboratory, before the fibril growth technology was 
transferred to The Carborundum Company. The description of dispersing and aligning the fibrils through flow of the dispersant fluid was adapted for trial testing, and the excellent orientation potential was confirmed.

Encouraged by the success in dispersing and aligning the fibrils by the wet-laid method, the contract deliverables were changed to be completed using the wet-laid system.

The following report details the work performed chronologically for the three different processing methods.

\section{Traditional Textile Fiber Processing Methods}

The work began with the establishment of a negatively pressured environmental isolation booth in order to provide a safe working environment with the fibrils. In the four-month booth installation delivery time, preliminary work was completed. A literature search was performed for information on asbestos yarn processing, air-vortex spinning, and potential processing alternatives. A visit to the Carborundum facility in Niagara Falls was arranged to learn about the fibril growth process and observe fibril harvesting techniques. Potential ways were discussed on how to integrate the Carborundum fibril production and textile processing alternatives to the traditional textile method (Appendix 2). Chopped Nicalon was procured as a model fiber and textile equipment was purchased for modification and use in processing.

\subsection{Model Fiber - Nicalon SiC}

Because of the experimental nature of the fibril growth process, a limited amount of fibrils was available for this program (180 grams). For this reason and because of the empirical nature of the work, it was determined that model fibers would be used to establish initial processing parameters. Chopped Nicalon was chosen as the closest commercially available fiber to model the high modulus and fiber diameter of the fibrils (Appendix 3). In chopped form Nicalon is entangled to a degree similar to a mass of harvested fibrils. By using Nicalon as a model fiber, processing parameters would be established and the potential for success in processing the fibrils would be extrapolated from the results. 


\subsection{Experimental}

Experiments in traditional textile processing concentrated on the carding process and air-vortex spinning. The carding process is the point in the system which provides the separation and fibril orientation required in the final yarn and must precede other processing steps. Once the carding operation is completed, the spinning operation is the next important processing step to consider.

The first pieces of equipment procured were two machines to be used for carding. One was a developmental card for future production work and one a miniature machine suitable for laboratory scale production work. Hand-carding equipment was purchased for initial experimentation in carding parameters of carding surfaces, fiber blending, etc. This first work to determine the parameters of carding operations, through hand carding experiments, could not begin, however, until the environmental booth was installed.

Our study focused first on the long-term goals of assembling a complete system consisting of the modified miniature carding unit, the drafting machine, and the air-vortex spinning unit.

Of the different options available in spinning operations, it was determined that the air-vortex method would be the best choice for investigation because the apparatus offered a minimum of abrasive surfaces and was mechanically simple to fabricate.

\section{Textile Machinery Requirements for Yarn Manufacturing}

Textile processing of staple-length fibers into yarn requires, at a minimum, the following equipment: carding equipment, a drafting machine or zone, and a spinning machine.

Carding is one of the most important operations of the staple fiber processing system. ${ }^{8}$ It is at this stage that the primary amount of fiber separation and orientation occur. Further processing can enhance the orientation, but the carding operation is considered the base of the spinning process. If short fiber cannot be carded successfully, it will be difficult, if not impossible, to proceed with the yarn formation process.

${ }^{8}$ Zoltan S. Szolaki, High-Speed Carding and Continuous Card Feeding. Charlottesville, VA: Institute of Textile Technology, 1977, p. 1. 
Carding requires two matched parallel surfaces set with angled pins or points. These parallel surfaces can be in the form of a set of two hand cards (surfaced wooden paddles with handles) or at a production level in the form of surfaced cylinders set closely together.

Drafting is the textile term for attenuation. A rope (sliver) of carded fiber is gradually reduced in linear weight to a smaller rope (roving) through speed differentials of sets of rollers. A sliver is fed at one speed to a faster moving set of rollers; a "necking down" of the sliver is created. This is repeated multiple times dependent on the desired fineness of the finished yarn and to make the reduction gradual and thus preserve the quality of the yarn. Although special drafting machinery is utilized in production level manufacturing, drafting can also be effected by a single zone of speed differentials such as that found in hand spinning. In the latter case, one hand draws out or drafts a sliver as the other hand is feeding the result to the spinning wheel.

Spinning is the textile term for integration of the fiber mass into a cohesive, strong linear fiber assembly by inserting twist along the length. The twist serves to force the fibers into closer packing, introducing interfiber friction which allows transfers of stress from fiber to fiber, creating cohesion and therefore strength in the yarn. Twisting forces the fibers into a helical configuration relative to the yarn axis but plying multiple single yarns wother creates a yarn in which the fibers are oriented along the axis. Plying is the twisting of multiple identical yarns together in the opposite direction to the direction the component yarns were twisted. The opposite twisting directions cancel out and a torque-free product with fiber oriented along the yarn axis is created (Figure 9). Twist can be inserted by different methods. Ring spinning introduces twist by feeding the roving through the middle of a ring and under a small metal clip which travels around the ring, thus forcing the yarn to rotate around itself. Open-end spinning uses a rotor to spin roving. Spinning can also be achieved by feeding the roving into an air vortex created by evacuating air from a tube and drawing the twisted yarn from the opposite end. 


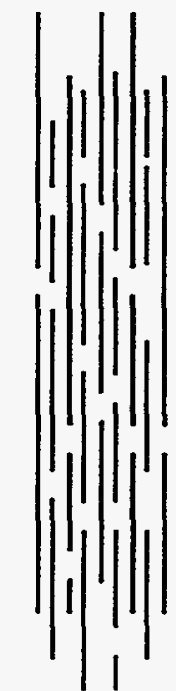

Fibers Axial in Untwisted Roving
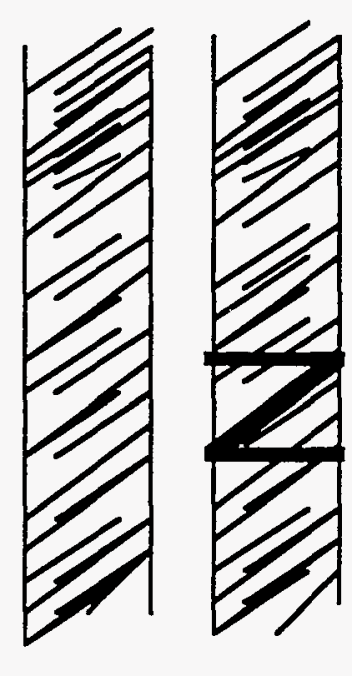

Fibers Helical in Two "Z" Twisted Yarns

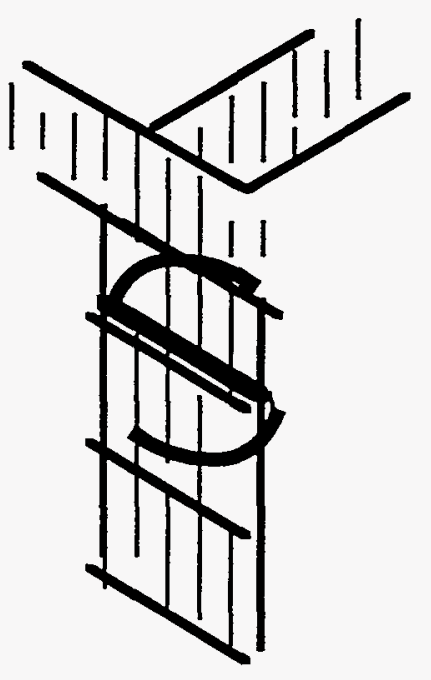

Fibers Axial in 2-Ply Yarn of Opposite "S" Twist

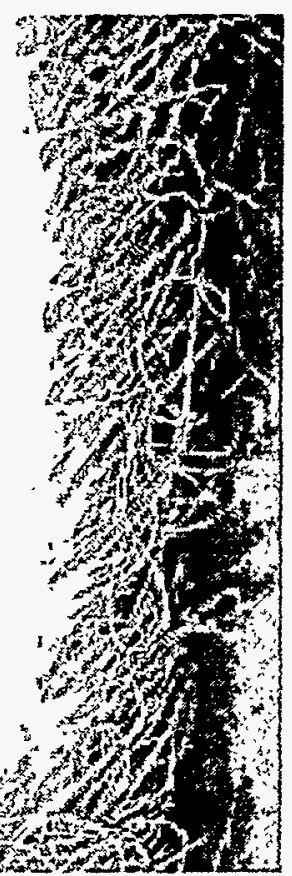

Figure 9. Fiber orientation at different stages of traditional textile processing and magnification of a spun yarn showing the helical configuration.

\section{Textile Machinery Modifications}

Consider the fact that used, miniature laboratory-size carding machines are rare and difficult to import. New imported miniature machines are rather expensive. Therefore, it was determined to modify an existing textile machine. The machine we procured for carding was originally a component of a specialty sample knitting machine and required substantial modification to be utilized as a functional carding machine. Supporting items required for the card included a 1/3 HP DC motor, motor speed controller, gears and bearings, and support bench. These were procured and the card was assembled. Additional missing items on the card were fabricated and added, including a doffer roll and comb, items which serve to transfer the carded fiber off the machine. These additions required mechanical modification work to achieve the proper speeds relative to the carding zone. Cotton fiber was successfully carded on the modified machine (Figure 10). 


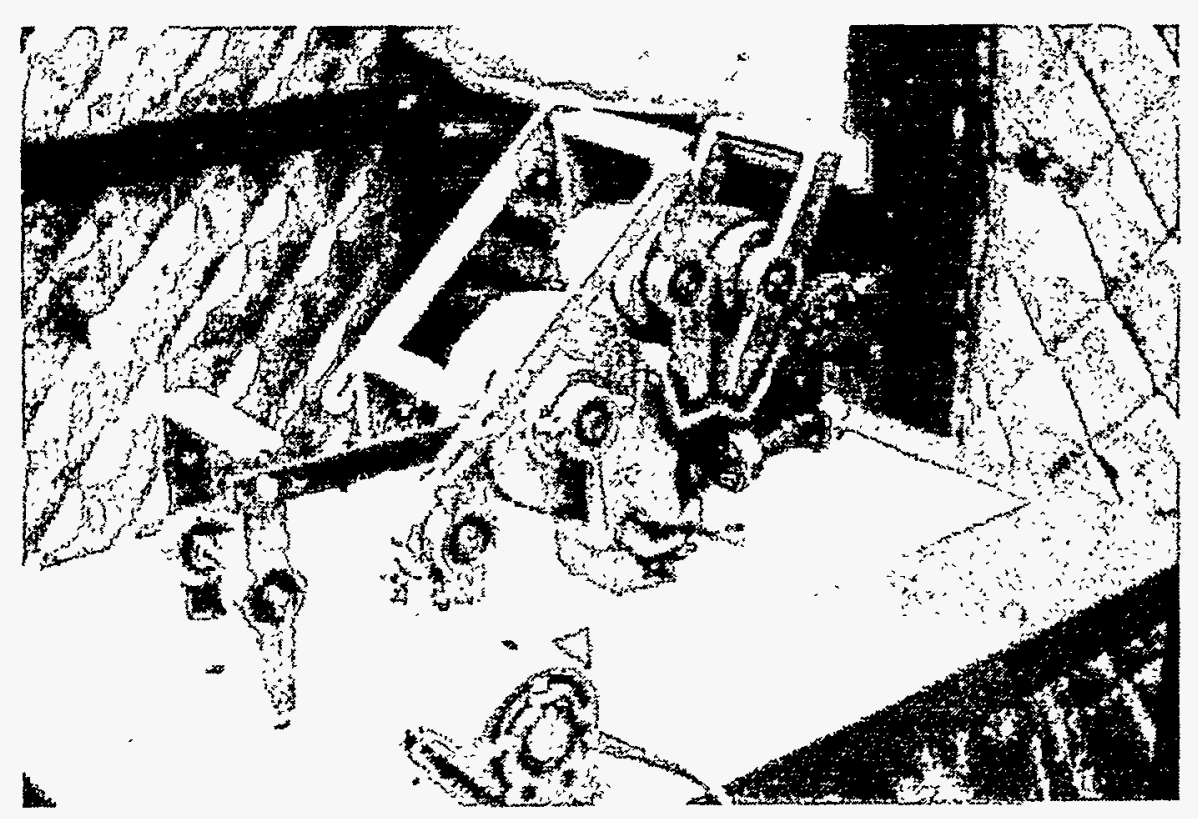

Figure 10. Modified miniature carding machine carding cotton fiber.

A drafting machine for carded fiber sliver was constructed using a small specialty fiber testing machine manufactured by Custom Scientific, Inc. This testing machine is designed to test interfiber cohesion by drafting a sliver or roving and making stress/strain measurements. Its drafting capability at a slow rate of speed makes it an ideal drafting machine for lab-scale work.

Parts were procured to assemble an air-vortex spinning machine. A glass tube with a secondary tube mounted on its side was fitted with a Venturi tube which creates a variable vacuum from controlled compressed air. A small pin-covered comb roll (on its own motor mounted on a specifically designed and machined bearing holder) was added to pluck and feed fibers from a carded roving into the air-vortex spinning tube. This comb roll is positioned between the drafting zone and the vortex tube. A set of take-up rolls on a separate motor was added to provide a controlled method of yarn delivery to compliment the controlled feed of fiber via the comb roll. Two criteria were necessary for the air-vortex method to work: a continuous length of yarn had to be formed and the yarn had to possess sufficient cohesion for processing.

A sample of cotton yarn was spun successfully on the completed spinning apparatus using cotton roving as a fiber source (Figure 11). A great deal of fiber waste (77\%) was collected from the vacuum filter after the yarn was made. This fiber waste occurred as 
fiber was fed to the air vortex but failed within the twist zone to twist around and become incorporated in the forming yarn. The fiber could be recycled by refeeding it to the carding system to realign.

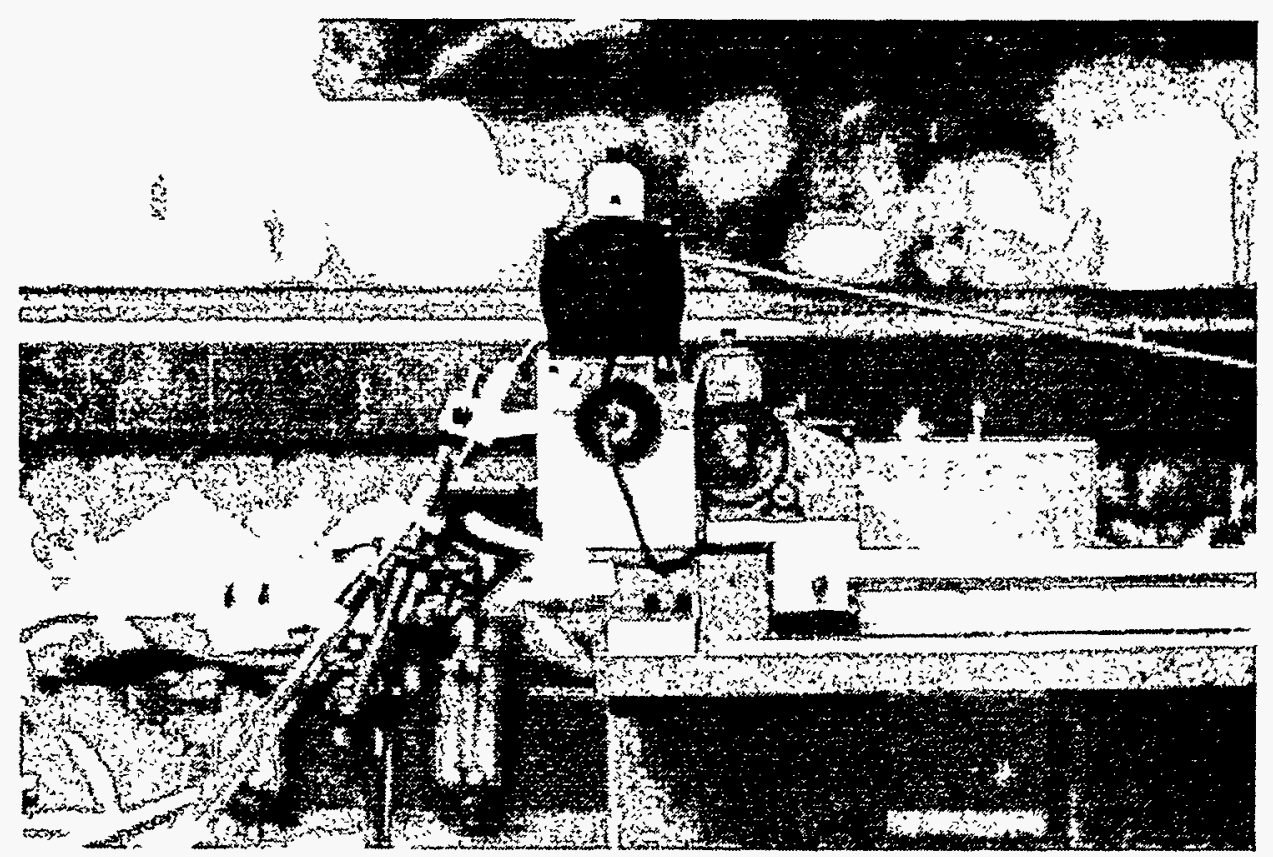

Figure 11. Side view of air-vortex spinning unit spinning cotton roving into yarn.

\subsubsection{Hand-Carding Experiments}

After the environmental booth installation was completed, hand-carding experiments began using Nicalon and carrier fibers. The hand-carding experiment was designed to identify the critical processing parameters and the auxiliary tools required in the construction of the miniature laboratory carding machine.

Initially, a set of commercially available hand cards were obtained. These hand cards, which come in pairs, are wooden platforms mounted on handles. Covering the wooden platforms is a $n$ array of wire points which is called the card clothing.

On these hand cards the array of wires is specifically called a fillet clothing type, meaning that the clothing (fiber processing surface) is made by punching fine wire staples through a tough, thick fabric laminate backing. The wire staples are bent at an angle, giving 
two specific directions to the card clothing, point or back (referring to the leading edge of the wire staple.) When fiber is placed between the two card surfaces and the two cards moved in opposite directions (point-to-point orientation of the clothing), the carding action takes place. The point-to-point orientation serves to tease and separate the fibers apart and orient them. A point-to-back orientation of the two carding surfaces, which occurs when they are moved in the same direction, causes the fiber to be transferred from one surface to the other (Figure 12). These two relative motions of the hand cards are created simply by changing the positions of the hands. At a production level, cylinders are covered with clothing oriented in the two different directions, allowing carding and transferring to occur continuously.

The hand cards used in our study had a clothing population of 240 points/in 2 . In experiments these hand cards could only card wool fiber because of this low wire density. Wool fiber, which is a highly crimped, long fiber, can be carded on a low clothing density because there is sufficient length and crimp in the fiber to bridge the distances between the points. To card the model fiber Nicalon alone and in blends with cotton requires card clothing with a higher density so that the shorter, finer cotton fiber and short, crimpless Nicalon could bridge the shorter distances between the points.

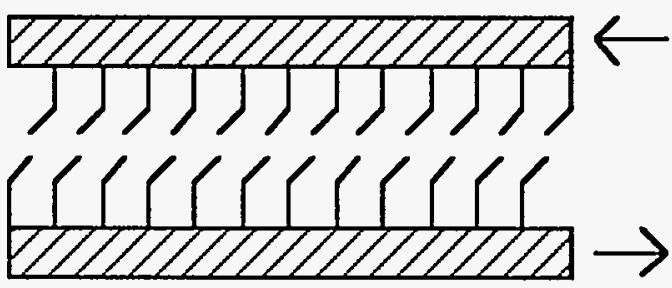

a) Point to point orientation of card clothing yields fiber carding action

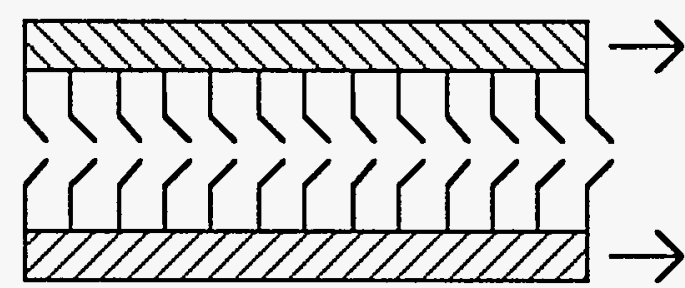

b) Point to back orientation of card clothing yields fiber transfer action

Figure 12. Movements and actions of carding surfaces. 
In order to increase the wire density, fillet-type card clothing with 460 wire points/in ${ }^{2}$ was obtained, the highest wire density available in the fillet type of clothing. Since the card clothing was not available on hand cards, two surfaces were built in the form of a stationary carding box and double-handled moving card surface and covered with the new clothing. The box was built su that shims could be used to accurately adjust the carding parameter--the distance between the two carding surfaces (Figure 13).
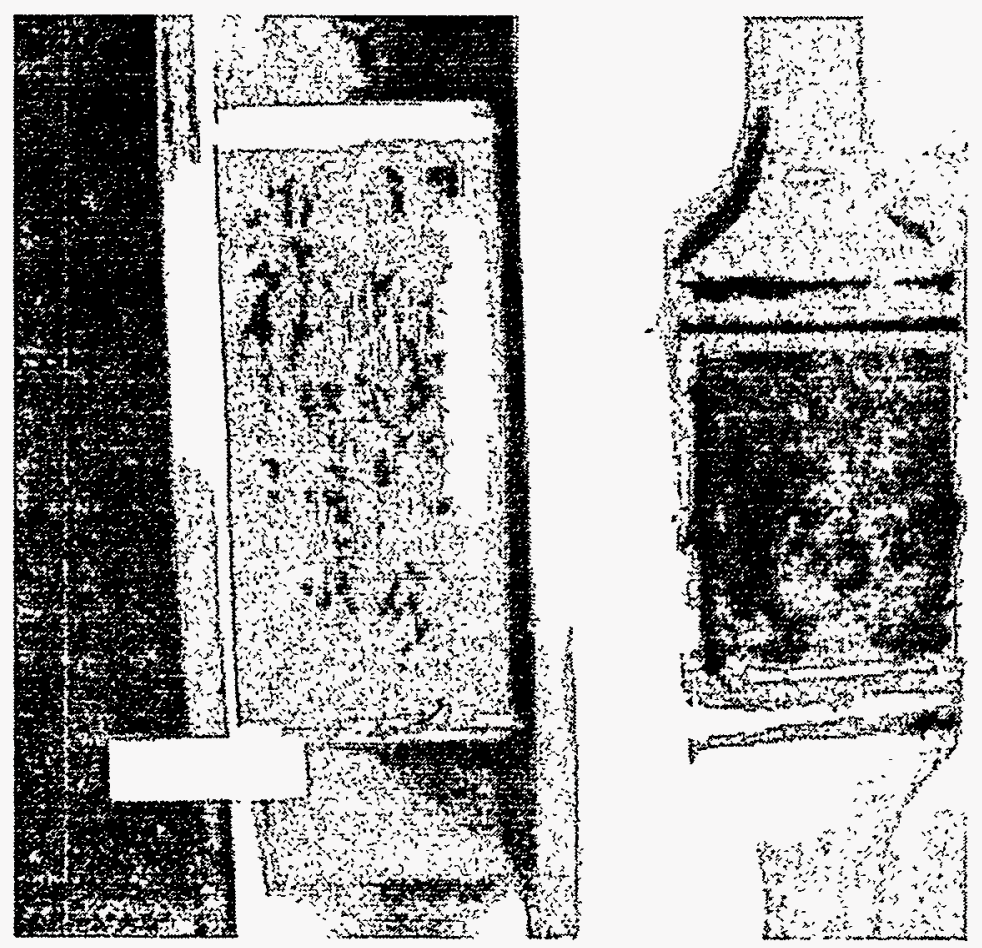

Figure 13. Fillet clothing covered carding box with matching surface.

A series of experiments was carried out wherein blends of cotton and Nicalon, ranging in 10 percent increments from cotton at 100 percent to Nicalon at 100 percent were carded using the carding box (Appendix 5). At each blend level, the results of carding the Nicalon fiber were not satisfactory. The fine, short, uncrimped Nicalon fibers would fall between the points of the card while the cotton, as a longer, coarser, crimped carrier fiber, would stay on the wires of the card and be carded. The end result was that the Nicalon received a minimum of the carding action of the wire points, as it sunk below the surface of the points. Nicalon dust was created by 
the action of the pins on the high modulus fiber before it sank below the points.

The fillet card : clothing proved to be too harsh for the Nicalon fiber. These difficulties were attributed to the lack of crimp, the high modulus, and the short length of the Nicalon fiber. Crimp in a fiber allow: a wire point on one card face to grip it as points on the opposite card face work to pull against that grip. Nicalon fiber at a 100 percent level could not be carded as the point faces brushing each other would not hold the fiber but instead abraded and broke the high modulus fiber before wedging it down below the level of the points. The fiber had to be scraped out of the wire points. As the amount of cotton fiber in the blend was increased, it was hoped that the cotton fiber would support and carry the Nicalon and the Nicalon would be carried along with it. It is well know in textile fiber processing that fiber crimp to necessary in order to achieve intimate blending of two fibers. The crimpless Nicalon could not grip and be held by the cotton and thus could not be exposed to the carding action. Because of this lack of cohesion between cotton and Nicalon fibers, there was no blending between the two fibers.

The modulus and length of the Nicalon also contributed to the carding difficulties with the standard card clothing. The short, highmodulus Nicalon acted as small rigid rods, unable to conform to the crimp of the carrier fiber. This is what appears to be the difference between processing the Nicalon in blends and processing asbestos in blends. Although asbestos also does not have crimp, it has several advantages over Nicalon in terms of traditional textile processing. First, in fiber form it does not exist in its finest discrete crystalline structure. Processed asbestos is frequently a bundle of smaller component fibers. These bundles can have striations and fringed end $s^{9}$ which may act as crimp substitutes.

Alternatives to the fillet card clothing were examined. It was decided to test whether the Nicalon could be oriented to some degree or if it could be forced to stay on the surface of some other type of card points. For this it was necessary to produce a higher density of pins which could be dense enough to support the Nicalon and prevent it from sinking below the point levels.

${ }^{9}$ Ernest R. Kaswell. Wellington Sears Handbonk of Industrial Textiles. Wellington Sears Company, 1963. p. 319. 


\section{Alternative Card Clothing}

A search proceeded for other materials to use as card clothing, materials which would feature more points/in ${ }^{2}$ and keep the Nicalon from falling between the points.

Metallic clothing was the first new option explored. Metallic clothing is another production type of card clothing, which is made of a sawtooth-shaped ribbon of metal which can result in higher populations (up to $800 / \mathrm{in}^{2}$ ) because of the stability of the ribbon. It is applied on-edge around the circumference of a drum. A hand version of metallic carding does not exist but a facsimile using saw blades set on edge and in parallel alignment does. This was done for two reasons. First, it is a greater number of points/in ${ }^{2}$ needed than is available in metallic clothing; secondly, the metallic clothing is difficult to assemble into a small hand-carding unit because the very narrow uncut metal base of the ribbon makes it difficult to assemble except by winding onto a drum, under tension, etc. There was no success carding with this jig. The width of the teeth did not allow for the required fine penetration between. the fine Nicalon fibers (Appendix 6).

Pile structure fabrics were examined next as potential carding surfaces. Pile or plush fabrics in many styles are used in apparel and furnishings applications, and are commonly known as a velvet or velveteen. They all feature a vertical element essentially perpendicular to the textile plane, similar to the orientation of the points in traditional card clothing. However, the pile is formed of tufts of multifilament yarns or spun yarns, which "open" after processing to expose the ends of discrete, fine fibers. The "population" then becomes much higher, being composed of these fiber ends. The appearance of the textile is that the backing fabric is completely obscured because of the density of the pile. This density was perceived as being able to support the fibrils without any fallout below the level of the pile tips. Because pile fabrics have a nap, or slight directional orientation to one side, they can offer the same necessary opposing orientation as standard card clothing. In addition, because the opened fiber ends are much finer than the wire or metallic style points, there was the possibility of a carding action penetration into a tangled fine fiber mass. It was thought that the 
flexibility of the pile would be advantageous in gently controlling the fibrils.

Two pile fabrics were tested with Nicalon, the model fiber: a pile fabric obtained from a paint pad, and a pile fabric that came from lint brushes. Neither fabric was successful in a carding action. One reason is that the separated fibers of the pile were too flexible to translate enough force to separate the fibers. The second reason is that the tufts of opened fibers still worked together as a single unit. At the point the tuft disappears into the backing fabric, the cut yarn pile is compressed into a flattened round cross-section; but as it opens up into discrete fibers, the fibers form a fan shape, which effectively works as a single chisel unit instead of as separate units (Appendix 6).

\section{Carding Process Conclusion}

Fibers which have been traditionally carded have the length, firmness, crimp, and elastic properties which allow them to be teased into a straight alignment through contact with a set of metal point surfaces. These same properties are not found in the Nicalon fiber nor the SiC fibrils.

It was concluded that carding Nicalon, if possible, would require considerable card clothing development. This requires a very fine, stiff material for the card clothing. This material would nave to be applied to a backing in a dense population, protrude in the $z$-axis, be flexible enough to give slightly under pressure, yet stiff enough to hold a directional orientation, and be tough enough to withstand the abrasion of the carding action. Developing a material for this application would take a great amount of effort even if this were considered a promising avenue. But the poor results of carding the Nicalon led us to the decision of abandoning carding as an appropriate process for the fibrils. On the basis of the Nicalon experiment, it was concluded that the higher modulus, larger diameter, crimp fibril would not survive the carding process.

\subsubsection{Air-Vortex Spinning}

Once the air-vortex spinning apparatus was proven to work with cotton fiber, efforts were made to incorporate Nicalon into a cotton roving to test the spinning properties of the Nicalon. This was 
necessary because an oriented, cohesive Nicalon fiber roving was not successfully produced through the carding process.

The cotton/Nicalon blend was fed into the air vortex and the resulting yarn qualitatively analyzed. The yarn had poor uniformity and was comprised primarily of cotton with spots of Nicalon appearing randomly. The Nicalon which appeared in the yarn reflected the orientation of the Nicalon fed in (i.e., a random orientation). Places in the yarn where Nicalon was predominant were weak and could be easily pulled apart. There was the same high waste factor of fiber (68 percent) collected in the vacuum filter as was collected spinning cotton fiber alone. The important difference between spinning cotton and the cotton/Nicalon blend was that the waste Nicalon fibers were significantly shortened in length to the extent that they could not be reused by redistributing the shortened fiber on another roving. This broken fiber was caused at several stages. First, the transfer of the fiber from the feed roll to the air-vortex feed tube zone causes the fiber to bend to a high degree as it is pulled free (Figure 14).

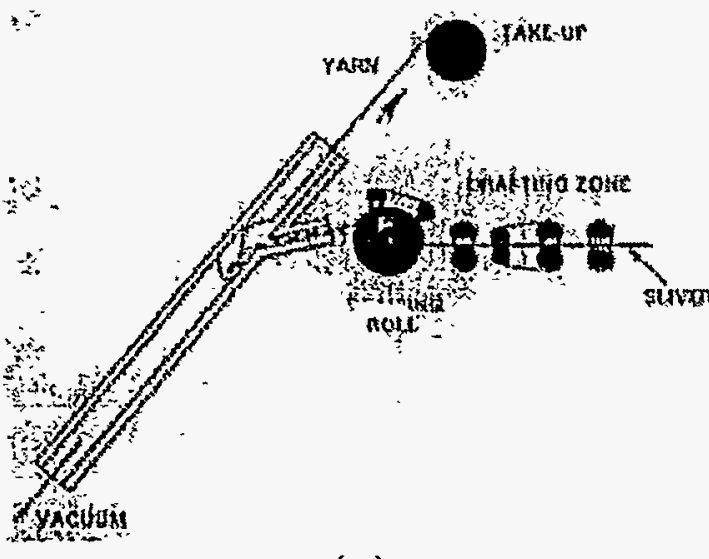

(a)

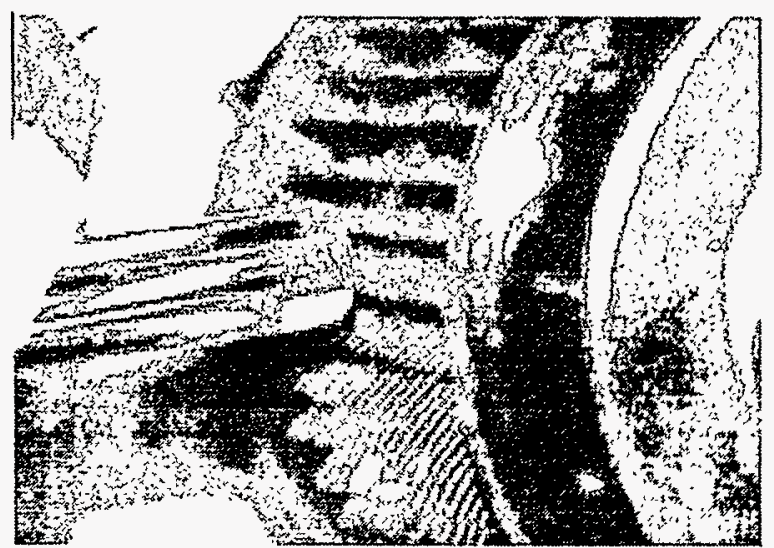

(b)

Figure 14. (a) Schematic of air-vortex spinning; (b) Transfer point of fiber from feed roll into vacuum feed tube of air-vortex spinning.

The additional observation was made that the yarn formation within the vortex also requires the fibers to bend to a high degree to create the twist. These two points in the processing, which require high bending, make the spinning process unacceptable. 
Spinning Process Conclusion

Spinning was concluded to be an inappropriate processing step for chopped Nicalon, and thus, the higher modulus fibrils because of the severe fiber bending the fibril will encounter using this process.

\subsection{Summary}

The two processes of carding and spinning were judged inappropriate for processing the $\mathrm{SiC}$ fibrils based on trials with processing Nicalon fiber as a model fiber. The crimpless character of the fibers prohibited the adequate blending with other traditional carrier fibers necessary for processing continuities. The high modulus fibers were susceptible to high rates of breakage in the processing where bending of the fiber was necessary.

The results of the traditional textile experiments led to more specific requirements for the four processing steps and the subsequent modification of the contract statement of work.

\section{Nontraditional Textile Processing Methods}

Based on the study of traditional textile fiber processes, it was concluded that $\mathrm{SiC}$ fibrils are significantly different from the traditional textile fibrous materials in their geometrical and mechanical properties. It was therefore concluded that a new method for fibril separation and alignment was required, one which could minimize fibril abrasion and breakage. Fibril bending and twisting had to be minimized in processing to prevent fibril breakage, thus eliminating any mechanical means of integration. An auxiliary binder system was envisioned as the alternative to interfiber cohesion provided by twisted, crimped fibers.

Two nontraditional textile processing systems, the adhesive-tape method and wet-laid fluid method, were designed for experimentation. 


\subsection{Adhesive-Tape Process}

\subsubsection{Introduction}

During an early program visit to Carborundum, alternatives to traditional textile processing were discussed (Appendix 1). From those discussions came an early idea to convert fibrils directly from the growth plate into yarn. Fibril orientation would proceed on fibrils in their growth pattern, and the oriented fibrils would be integrated by applying adhesive tape. The method was accepted for work under the statement of work issued in June.

The potential advantages to the adhesive-tape approach can be summarized as follows:

1) Orientation would be easier for two reasons. First, less fibril entanglement would be present on the plates than in harvested fibrils. Secondly, the fibrils as grown would be anchored to the plate by the catalyst balls, allowing orienting tools better working action.

2) Fibril breakage would be greatly reduced if the fibrils could be more easily oriented.

3 ) Virtually all of the fibrils grown on a plate would be utilized, therefore reducing waste.

4) Fibril integration would be achieved through an adhesive tape; therefore, fibril bending would not be critical to yarn formation.

5) The adhesive-tape substrate would be sufficiently flexible for further processing.

6) The health hazards would possibly be reduced since fibrils are encapsulated.

\section{Initial Adhesive-Tape Concept Model}

To demonstrate the initial concept of direct conversion of the fibril, a tape sample was made using Nicalon yarn. A free end of the yarn was adhered to the tape and cut to length approximating growth length of fibrils. This was repeated multiple times to form a 
shingled effect of one row of axially oriented fiber overlapping another row for complete coverage. The shingling allowed the tape to bend readily despite the high modulus of the Nicalon. Of the completed fiber/tape assembly, the fiber constituted 78 percent by weight. Although this high fiber loading and axial alignment made the sample a highly stylized model, it was demonstrative of the concept.

\section{Fibril Plate Scheduling}

The graphite fibril growth plates measure 12 inches by 6.25 inches. To transport and store the plates, Carborundum and APD both built wooden shipping crates. These featured narrow slots milled in three sides to keep them rigid and in parallel formation to prevent motion or impact or crushing of the fibril growth which occurs on one or both sides of the plate. The top of a small crate served as an elevated work station to access one side of a plate without crushing the fibril growth on the opposite side.

A fibril plate delivery and return schedule was developed in order to maintain the contracting of work at APD and Carborundum.

\section{Fibril Plate Growth Variations}

Fibril plates used in orientation experiments could be divided into four different growth types: 1) baseline, 2) baseline-less dense, 3) short, and 4) shortest.

An example of the baseline growth is shown in Figure 15. The length of fibrils is approximately $1-2 \mathrm{~cm}$. The areas which appear white are places where the fibrils have grown long enough in the furnace to hit a neighboring growth plate, creating a mass of irregular submicron diameter fibril growth. A close-up of this plate growth can be seen in Figure 16, revealing the dense entangled growth. 


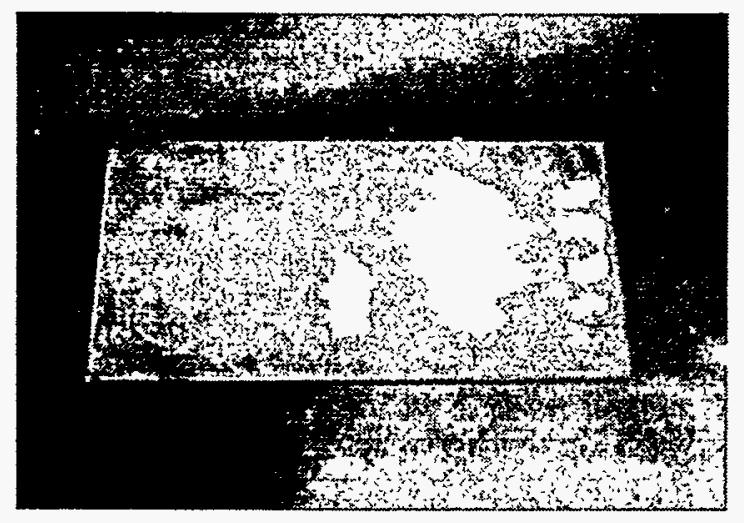

Figure 15. Baseline fibril growth plate.

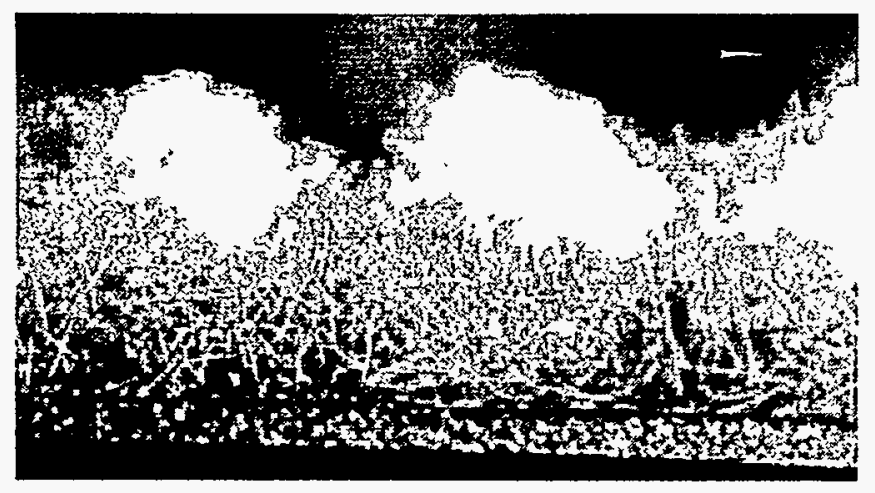

Figure 16. Close-up of the baseline fibril growth seen in Figure 15.

\subsubsection{Separation and Alignment}

Brushes, combs, fluid flow, and particle flow were evaluated as tools for the orientation of the fibrils.

Different types of brush bristles and combs in different lengths, densities, and fineness were used in experiments to identify a tool which could penetrate the fibril mass and separate and align the fibrils.

Brushes of all types were tested by moving a brush along the surface of the fibril growth parallel to the plate surface. This technique resulted only in knocking over and flattening the fibrils through pressure because all the brushes had bristles which were too soft, flexible, and course to penetrate the fibril mass. The brush bristles thus acted like a bulk mass running over the fibrils. 
Trimming the brush bristles to shorter, stiffer lengths and removing bristles to create individual bristle "spikes" did not improve the brushes as tools.

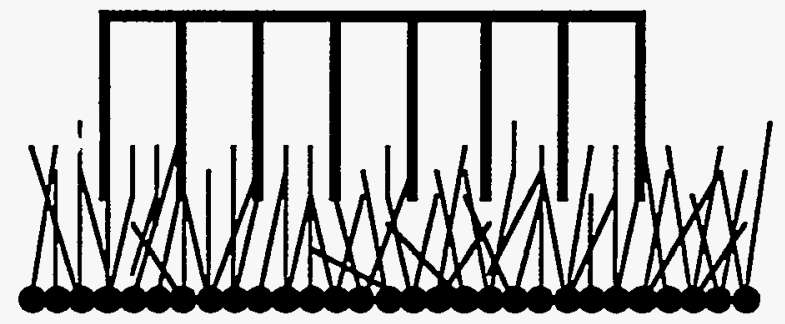

a)

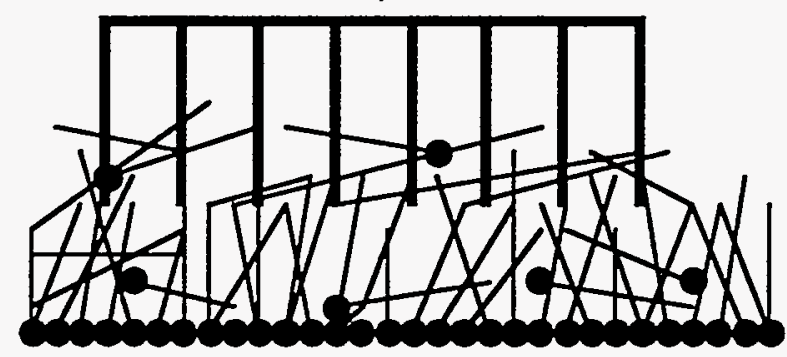

b)

Figure 17. Schematic of a) ideal combing action if fibrils remain anchored to the plate, and b) raking action of fibrils as they break or are lifted from plate.

A variety of combs were made using needles and pins of different sizes and densities when punched through a backing. Applied to the plates along the fibril growth surface in the same manner as the brushes, the pins were stiff enough to penetrate the fibril mass but they were not fine enough to do anything more than create a series of gouge lines in the fibril growth. As the pins hit fibril entanglement, they snagged and knocked over the fibrils. This was true regardless of the fineness of the comb teeth. It was attributed to: 1) the low strength and elasticity of the bond between the fibrils and the plate and 2) the fibril entanglement. The comb may rake them. The difference is that in combing the attached fibrils get oriented perpendicular to the row of comb teeth, but in raking the broken fibrils move freely and get separated out and oriented parallel to the row of comb teeth. Because of the fibril entanglement, these two actions are occurring simultaneously with any given stroke of a comb. As a result, the fibrils are broken and they tend to break other fibrils when they move freely (Figure 17).

Although Figure 17 is merely a schematic, the relative scale of the comb pins and the fibril diameters in the figure accurately 
represents another problem: the relatively thick comb pins could not finely separate the much finer fibrils, and the spacing between them. Each comb pin would thus "catch" or "snag" a group of entangled fibrils.

A rotary comb was assembled as a potential tool to prevent these divergent dual actions of combing and raking. A long flexible tape with inserted pins was spirally wrapped around a cylindrical shaft. By rotating this shaft over blocks on either side of the plate, the spiral configuration allowed a single pin to dip into the fibril growth at any one time and point. This prevented a raking action. The comb was gradually indexed down into the growth as the shaft rotated.

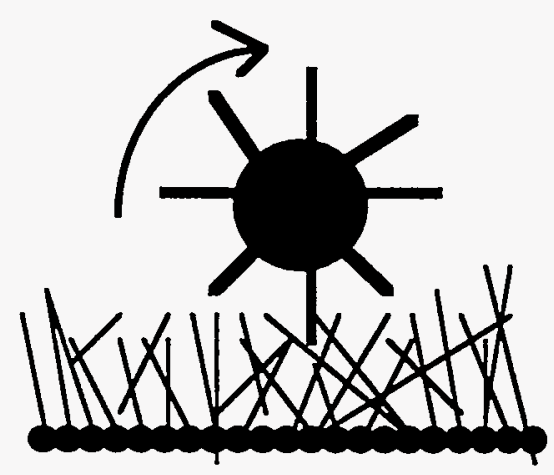

Figure 18. Schematic of a rotary comb.

The results of using the rotary comb were not much of an improvement. The pins would occasionally lift fibrils out of the growth and the rotating action would knock fibrils over as the indexing lowered the comb into the mass but otherwise the single pin at a time would have no discernible orienting effect.

In order to create a gentle motion, different fluids were applied to the plates with the effect of merely knocking over the fibril growth. Pressurized air, glycerin, and water all flattened the growth quickly. Fibrils of one plate were misted with water and then with air to create moisture droplets which could provide some orienting weight with directional air flow. None of the fluids oriented the fibrils to any discernible degree.

Particulates in the flow were the last attempts made in orienting fibrils on the growth plates. It was thought that perhaps fine particles could get between the fine fibrils and by forcing the 
particles to move in one direction could have an orienting effect. Table salt was used on plates vertically oriented such that the salt would travel down the plate under gravity. The salt did flow down the plate when it did not get trapped in the fibril entanglement but that which flowed had no effect on the plate. It didn't seem to flatten the fibrils nor to cause any orienting effects. The next particulate used was a much finer magnetic powder, which was hoped would provide some orientation under the application of a magnet to make the powder move. The powder moved, but some fibrils were pulled off the plate and mixed in with the powder. No orientation of the remaining fibrils was evident. Feedback from Carborundum indicated that the magnetic powder used on the plates could interfere with future use of the plate to grow fibrils. Photographs of fibril orienting efforts are found in Appendix 5.

\subsubsection{Catalyst Ball Removal and Adhesive-Tape Integration}

During early experiments in the adhesive-tape method, it was learned that the fibrils had to be leached of their catalyst balls by a strong acid prior to consolidation in a composite. This information had many implications for the adhesive-tape method.

The catalyst balls could not be removed from the plate before orientation for two reasons. First, the submersion in a liquid solution would flatten or mat the fibril growth, defeating the primary purpose of working directly from the plate. Secondly, an entire growth plate could not be submerged in the strong acid for catalyst ball removal for safety reasons (it would be unsafe to handle a large bath of acid to dip the plates). The catalyst balls would have to be removed at a later stage in the process (i.e., after orientation and tape application). However, laminating an adhesive tape on either side of the oriented fibrils would be problematic to the removal of the fibril catalyst balls because access of the leaching solution would be blocked to the fibrils through the laminated faces. This implied a single-sided adhesive-tape application, leaving an open side for acid access. The adhesive tape requirements became more stringent as the tape now had to survive the leaching environment while maintaining an adhesive hold on the fibrils before being burned out cleanly after lay-up.

Tape vendors were contacted with the list of requirements and subsequently a selection of open-sided fibril tape samples were 
produced and forwarded to Carborundum for leaching and burn-out tests. A woven fibril tape sample was produced to test whether the woven tape interlacing would provide sufficient mechanical integrity to keep the fibrils in position through leaching (Figure 19).

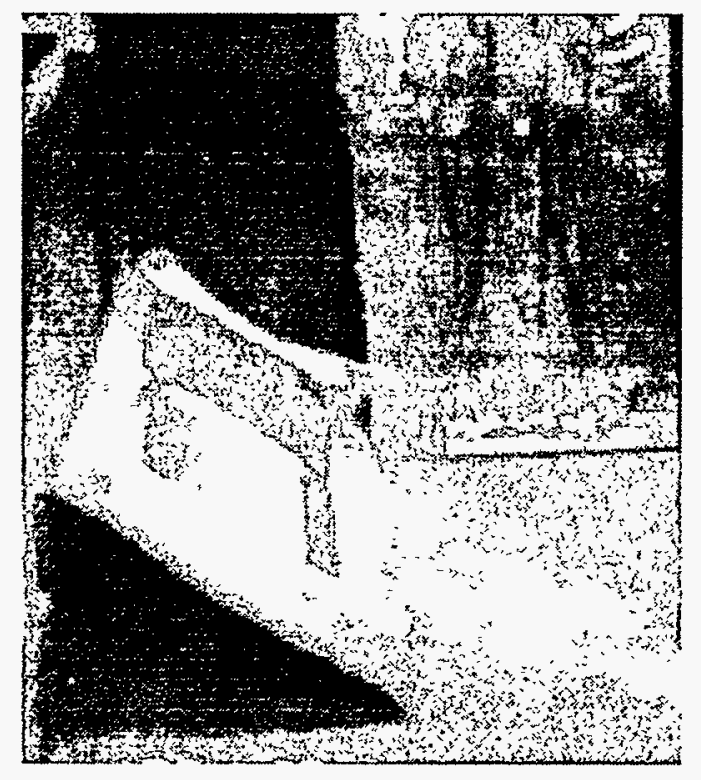

Figure 19. Tape setup shown at left (Section 5.7) being used to weave open-sided tape sample on cardboard loom at the right.

The best sample to survive the leaching environment with fibrils still intact on the adhesive and successfully burned out was a $3 \mathrm{M}$ brand tape marketed for metal plating applications requiring stability through acidic etching operations. The woven tape sample was not successfully leached because the leaching solution could not access the fibrils in between the interlacings.

\subsubsection{Adhesive-Tape Application and Handling}

To provide the necessary linear fibril integration, an adhesive tape was applied to a fibril plate after the orientation was completed. The application of the tape presented additional requirements and challenges.

The first requirement for applying adhesive tape is that the fibrils must be flattened or knocked over such that they are lying on the plate and not standing upright. This flattening step is required 
because once flattened, the plate presents a planar surface to which the adhesive film plane can be applied to get maximum adhesion. Tape could not be applied to fibrils left standing upright. Although most fibrils were flattened during the orienting process; additional efforts are often required in order to flatten the back fibrils. This flattening process was achieved by rolling over the fibrils with a metal rod. This action would result in a fibril mat with a different orientation than initially presented.

Once the fibrils were flattened, an integrating adhesive tape could be applied. After applying the tape to the fibril mat, pressure would be applied to make the fibrils adhere and the tape was removed from the plate. Ideally, the tape would cleanly remove the fibrils completely from the plate but this did not always occur. Patches of fibrils would occasionally remain on the plates. There was no clear pattern to this failure to adhere and remove fibrils. The appearance of the fibrils once transferred to the tape was that of a clinging, shaggy mass. Fibrils could be easily dislodged, and it appeared that much of the adhesion was due to interfibril entanglement rather than to adhesive bonding between each fibril and the tape (Figure 20).

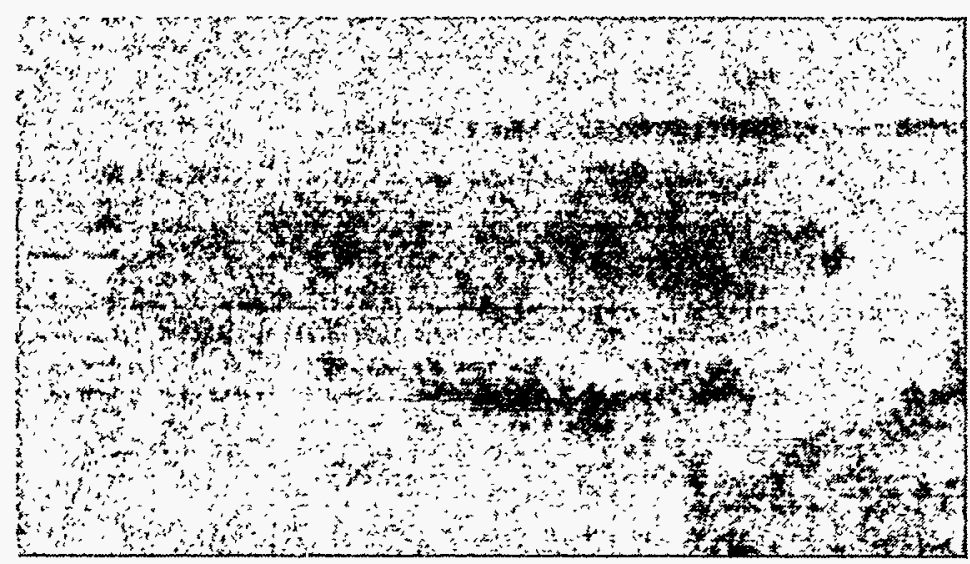

Figure 20. Tape setup showing uneven adhesive pickup of fibrils.

The next challenge was in slitting the tape to create the desirable yarn-like widths. The original concept was to apply a tape equal to the full width of the plate, laminate the fibrils with another tape layer, and then slit the tape to the desired width. Once the requirement for an open-sided tape was addressed, this concept became impractical. Slitting the tape while it was still affixed to the plate would potentially damage the plate by the slitting tool. Slitting the tape after removal from the plate could be performed in one of 
two awkward ways. One, the adhesive and fibril face of the tape could be applied down on a surface, potentially sticking and dropping fibrils, and then the tape could be slit; or two, the adhesive and fibril face could be $u p$ while slitting, requiring the sheet to be temporarily tacked down in order to keep it from sliding as it was slit. The adhesive would occasionally stick to the slitting tool causing drag on the tape, or the tool would pick up adhesive and cause it to pick up fibrils.

To avoid the potential difficulties encountered, pre-slit tapes were used to make a tape "setup." Multiple tape lengths were applied, adhesive face up, in close, parallel alignment onto a smoothfaced metal block the same size as the plate. The parallel tapes were held in position on the block with double-sided tape at the ends. After the orientation process, the tape setup block would be applied to the fibril plate and pressure applied to the back of the block to assure adhesive bonding of the tape to the fibrils. When removed, the block had an array of parallel, fibril-adhered tapes. The difficulty with this method is that the flattened fibril mat "bridges" across the division between tapes, effectively joining them through fibril entanglement. The solution was to cut between the tapes. However, this method led to the protrusion of fibrils off the tape edges.

After tape samples were made, the next challenge lay in handling the open-sided tape. In working with the fibrils, safe working conditions require workers to wear gloves, which stick easily to the open-sided tape. This problem can be solved by putting small tabs at either end of the tape samples. To facilitate transportation and weighing individual tape samples, holes were punched in the end tabs and brass office fasteners were used to hold the tabs onto wooden paint stir sticks, which are about the right length for the tape samples.

\subsubsection{Linear Density of Fibril Tapes}

The difficulties in completely clearing a plate through application and removal of adhesive tape led to samples with variable linear densities. These variations were also attributed to growth variations in the growth plates.

To test the growth variations, two growth plates were divided into one inch squares and each square was individually razor 
harvested and weighed. The resulting weight matrices of the plates is found in Appendix 6. From the matrices the natural growth variation can be seen, ranging from square to square by differences as high as 50 percent. This indicates the growth variation inherent in the furnace growth process.

\subsubsection{Summary}

The adhesive-tape method was judged unsuccessful because it fails to achieve the fibril orientation. Additional drawbacks existed in the subsequent processing. The application of tape was inefficient and did not always completely remove fibrils from the growth plate. The fibril growth is variable making it impossible to achieve uniform density. The open-sided tape samples were difficult to handle. A yarn made directly off a plate requires a leaching step to remove the catalyst balls. This in turn requires an open-sided structure to allow the accessibility of the leaching solution to the balls. From the experience of weaving with the open-sided tape samples, it is believed that additional textile processing would be extremely difficult. The open-sided tape samples cannot slide over each other because of some degree of exposed adhesive and because the fibrils on the tape give a rough, "shaggy" texture which does not promote compaction.

\subsection{Fluid-Based Processing}

\subsubsection{Introduction}

The use of fluid as a medium to process fiber is not new. The paper industry has used fluid flow successfully to make paper. One of the earliest attempts to use fluid to process fibrils was made by researchers in the Los Alamos National Laboratory (LANL) [ref.] The work reported by the LANL researchers was concentrated on the dispersion of the fibrils and their method was adopted in this study.

A preliminary experiment was performed in wet laying a small mat of $1 / 4$ inch long chopped Nicalon. New tools were purchased for this work, including a Buchner filter funnel, a flask to use as a vacuum trap for the funnel, and very fine stainless steel mesh (500 x 500 interstices/inch) trimmed to fit as a filtering screen inside the funnel. 
Chopped Nicalon was rinsed with acetone (to remove surface finishes) and dispersed in a 50/50 water/glycerin mixture using a magnetic stirrer. Full dispersion was not possible because of a residual finish left on the fiber; however, enough was dispersed to fill a funnel. The dispersion was filtered through the stainless steel mesh over the coarse-holed Buchner funnel using a vacuum. The vacuum is necessary both to create a controllal:c flow, and also because the mesh is so fine that the glycerin/water mixture did not flow rapidly through it. The flow of the dispersant during drainage was sufficient to cause the fibers in the dispersion to orient radially around each of the drainage holes in the funnel. This was a simple but elegant demonstration of the orienting flow phenomenon (Figure 21).

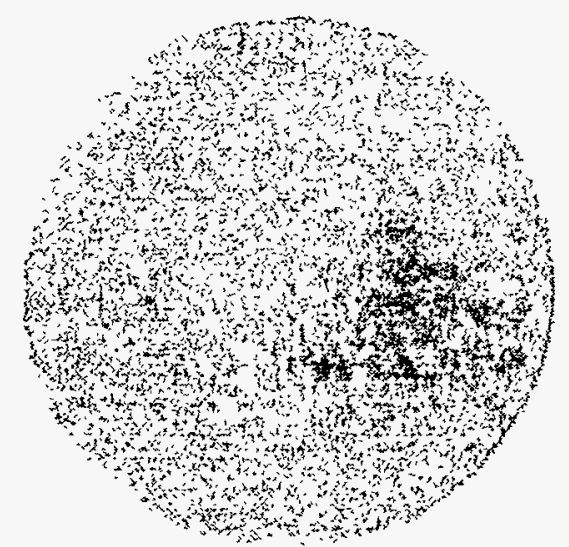

Figure 21. Nicalon radially oriented over drain holes.

The same experiment was repeated with harvested fibrils. First, the fibrils were dispersed in the same glycerin and water mixture which had successfully dispersed the Nicalon. The fibrils dispersed completely with no clumping together of fibrils as was seen with the Nicalon. The fibrils oriented radially on the screen around the holes of the funnel, indicating that the fibrils are orienting in the direction of flow. The small spots of wet-laid fibrils had no cohesion.

The radially oriented "fibril spots" produced in the Buchner funnel experiments are a good indication of the feasibility of fluidflow-induced fibril orientation but were insufficient to demonstrate the potential to create a linear, yarn-like product. Toward this demonstration, a small fixture for the funnel was designed to create a more linear orientation by directing the dispersed fibers or fibrils flowing down an angled channel. A small block of aluminum was cut 
to fit the Buchner funnel (approximately three inches long) and a $n$ angle milled along the top edge. The piece was cut in two, separated, and the resulting channel covered with screening. A piece of silicone sheet was cut to block all holes of the funnel except for the area of linear flow and placed first into the funnel with the aluminum fixture on top. When this assembly was seated in the funnel, the vaci:. $\mathrm{m}$ features were usable but localized, causing the flow of the dispersion to be forced down the channel (Figure 22).

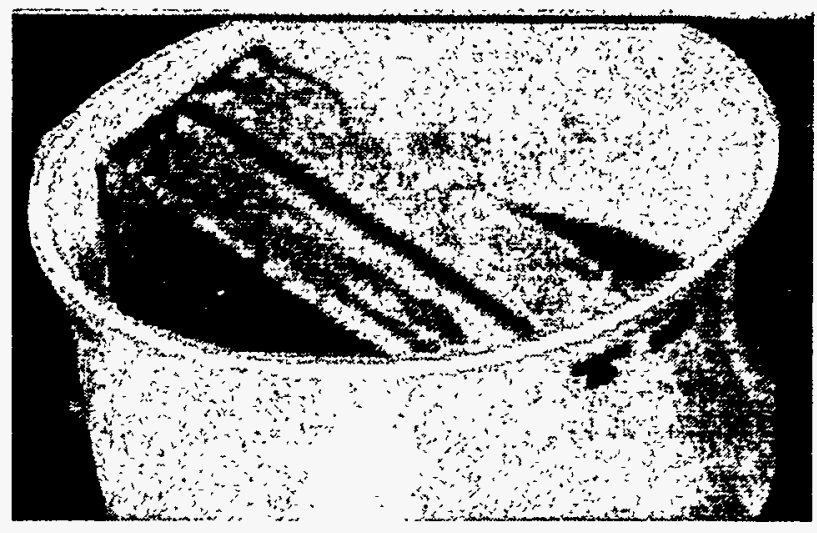

Figure 22. Initial linear flow fixture.

An initial test of the fixture was performed using dispersed Nicalon, hand poured from a beaker into the channel. The results were not good and ideas were generated to improve the concept. Several areas were identified as problems. First, if a blockage occurs in the flow, as when a single fiber gets wedged in the screening, the flow rapidly backs up with more fibers landing on the first jammed fiber. Secondly, there was no metering or continuity to the rate of flow. A variation of the fixture/funnel effort was tried in which a piece of aluminum sheet metal, bent to create a channel (but with no screening to snag fibers), was set up at an angle similar to the fixture. The fluid flow down this channel was directed onto a piece of screening with the channel being moved along the screen as the flow occurred (as in making a line with an ink pen). When the dispersed fiber flow would hit the screen at the bottom of the channel, the angle of the fibers hitting the screen would create the same sort of fiber jam as with the funnel fixture. The continuing flow would then form a puddle of disoriented fiber mixture. This is because the thickness of the dispersion medium causes it to sit on the fine screening when the vacuum suction is off. If the vacuum is turned on while the dispersion is poured, however, then the desired orienting flow is minimized because the vacuum acts too quickly on 
the liquid. It appears that, in order to be effective, the flow of the dispersion down the angled channel would have to be cut off at the source and at the end while a vacuum is applied simultaneously to "freeze" the oriented flow on the screen.

A solution was found in the relative speed rates of the channel movement and the dispersion flow. If the channel was moved at a faster rate (backward along a screen) than the dispersant flow, then the vacuum could remain on, to rapidly remove the dispersant, and the fibrils would be held in oriented position on the screen (Figure 23).
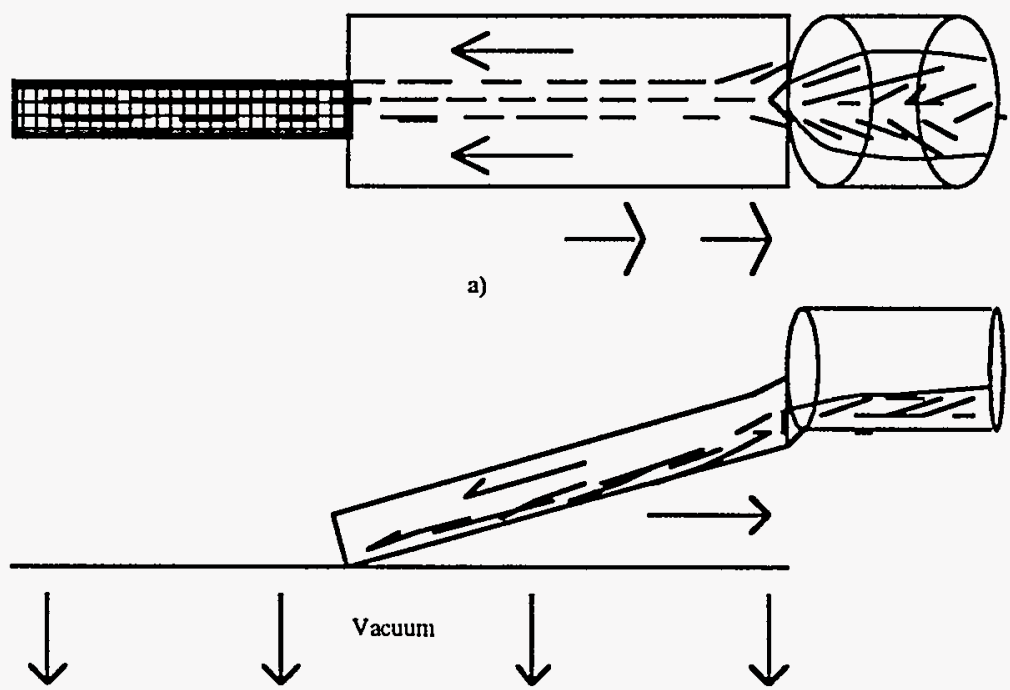

b)

Figure 23. Schematic of wet laying dispersed fibrils down a channel.
(a) top view;
(b) side view.

\subsubsection{Dispersion}

Dispersion is the process of suspending the fibrils in a liquid medium to the degree that they are individual, discrete bodies which can rotate and move freely as the liquid flows. This is the chemical equivalent of the mechanical opening process for fibers. Specific dispersion criteria was necessary for the laboratory-scale experiments which were performed. A high viscosity fluid was sought in order to promote hand-controlled flow. Because fibrils 
were weighed and then dispersed, 100 percent dispersion was sought in order to identify exactly the amount of fibrils in a given volume of dispersant.

Different dispersant fluids were tested for viscosity levels, fibril dispersion concentrations, and ease of flow. The best dispersant fluid was determined to be an $80 / 20$ glycerin/water mixture. It gave a slow rate of flow which could be easily hand controlled. Different fibril concentration levels in this dispersant were flow tested and the highest concentration was chosen which did not interfere with the flow. The concentration of fibrils in the $80 / 20$ glycerin/water mixture was 1.0 gram of fibrils dispersed in $2,500 \mathrm{ml}$ of the dispersant. Higher concentrations of fibrils had a "tumbling" type flow in which fibrils would not align but would instead seem to run into and tumble over other fibrils.

Initial dispersion efforts were easily performed. Harvested fibrils which had not been leached could be dispersed easily in a $n$ $80 / 20$ blend of glycerin and water. But to avoid the same difficulties encountered in the leaching process for the adhesive-tape method process preleached fibrils were used.

Difficulties in dispersion arose with leached and beneficiated fibrils, which took the form of tightly entangled masses. This can be attributed to the highly acidic leaching bath after which the fibrils are neutralized and dried. The change in $\mathrm{pH}$ values from the acid bath to the neutral batch results in fib-il "flocking" or "ilumping" together. This "clumping," when dried, results in a highly entangled mass very resistant to dispersion. One solution to this tight entanglement was to mechanically separate the fibrils with tweezers. This task, which needs further experimentation, was extremely time consuming and tedious.

From a dry, entangled ball of leached and beneficiated fibrils, very small tufts were removed using two pairs of tweezers. These tufts were dropped into the dispersing medium and would discretely and completely disperse after stirring for a minute. Tufts larger than approximately $1 / 8$ inch in diameter would not disperse (Figure 24).

Depending on the level of fibril entanglement, different batches of leached and beneficiated fibrils showed different levels of difficulty in dispersing the fibril. Some batches required a strong tugging action of the tweezers in order to pull off a small tuft from 
the larger mass of fibrils and others not as much. The more effort it took to pull off this small tuft the more likely it was that it would be difficult to disperse with a simple stirring action of the dispersant. In these batches, the fibril mass had to be reduced to very small tufts before dispersing.

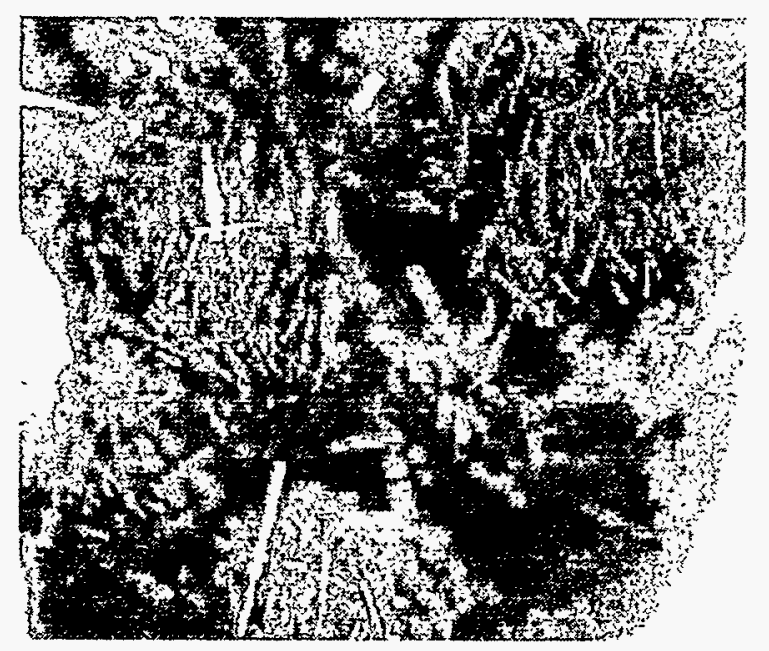

Figure 24. Close-up of fibril tufts before dispersing.

Because of this required tweezering action, it was difficult to understand how much fibril breakage was occurring as the fibrils were tweezered apart. Once the small tufts were in the dispersant, the level of f:bri! breakage was difficult to assess. It seemed that in the case of very highly entangled fibrils, breakage would occur when the end of a fibril embedded in the tuft cluster could not separate itself out when the opposite end was under force from the dispersion medium flowing past it. This left a tightly entangled center portion of shortened fibrils which could not be dispersed and had to be either discarded or even more finely tweezered apart.

Because of the slow rates of dispersing fibrils, it was suggested by the researchers from Carborundum to deliver the fibrils dispersed in an aqueous solution. The acidic leaching bath containing fibrils was raised to a neutral $\mathrm{pH}$ and this neutralized bath was sent to APD. But the flocking phenomenon had occurred with the change in the $\mathrm{pH}$ of the solution and these fibrils were not easier to disperse and required mechanical separation (tweezing) employed in the dry fibrils. Because they were wet they were more difficult to grasp and pull apart (Figure 25). 


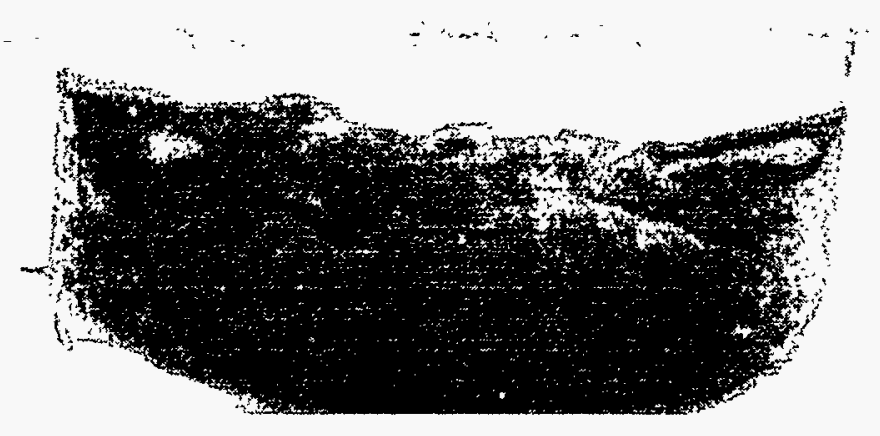

Figure 25. Fibrils in aqueous solution exhibit flocking phenomenon.

The dispersion problem was partially solved late in the program. At APD's suggestion, Carborundum began to dry the fibrils after leaching in what was termed the "paper" method, in a much thinner sheet of fibrils. These fibrils, while still requiring tweezers to pull apart, required much less effort, and in some cases took only a tenth of the time to disperse.

Once dispersed in the glycerin/water, the fibrils would settle to the bottom over time but could easily be redispersed by stirring for a short time. This particular dispersion medium seemed to prevent any reentanglement or clumping of the fibrils.

\subsubsection{Orientation and Accumulation}

Laying down the dispersed tibrils proceeded as in Figure 23. A small beaker of dispersed fibrils was poured, about $2 \mathrm{ml}$ at a time, down a sheet metal channel which was simultaneously withdrawn along a guide track on top of a vacuum screen. Because of the low levels of fibrils in the dispersant, this action would be repeated over 100 times in order to accumulate fibrils. When sufficient fibrils were accumulated, the movement of the channel would be repeated with plain water to rinse the fibrils of the trace amounts of glycerin. After this rinsing, the channel action would be repeated with a flow of a 50/50 PVA/water mixture which would remain on the fibrils as the assembly was removed from the screen. 


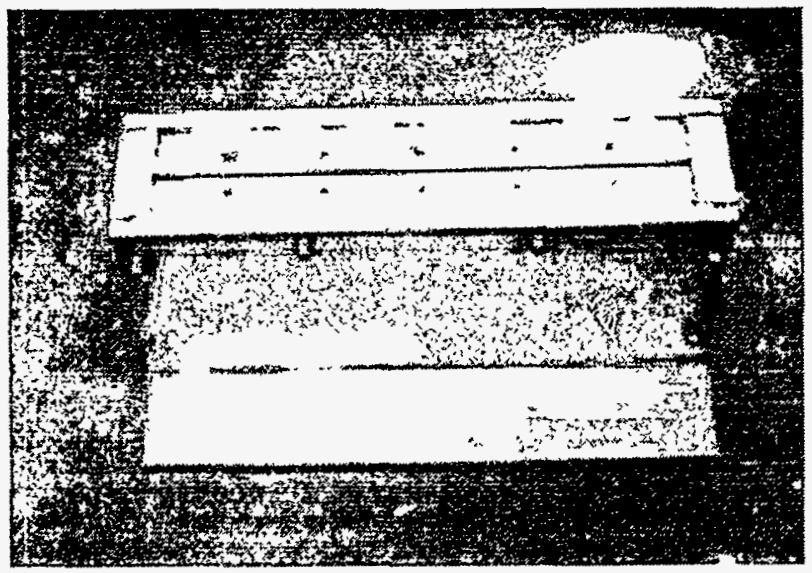

Figure 26. Aluminum wet-laying vacuum box with channel in center.

Figure 26 shows the first vacuum box built as a first scale-up design from the Buchner funnel fixture. The information generated subsequently about wet laying as a process came from experiments using this APD fibril alignment box (FAB). The shape of the channel was changed from a " $V$ " shape cross section to a square-bottomed shape because the "V" shape caused fibril wedging in the screen (Figure 27).

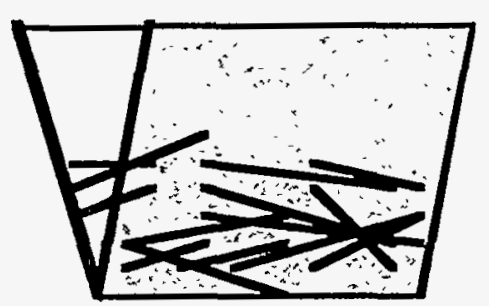

a)

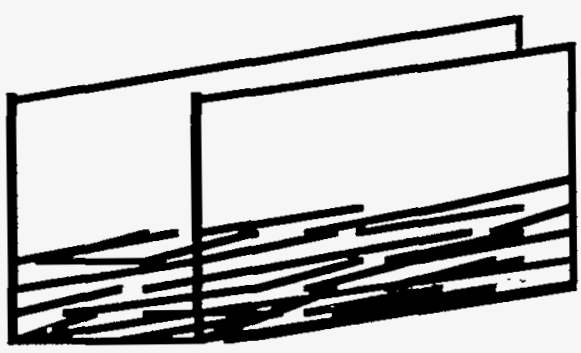

b)

Figure 27. Fibrils a) jam in increasingly narrow V-shaped cross section but b) do not jam in the square-bottomed cross section with screening on the bottom only.

The depth of the screen allowed an accumulation of fibrils to an almost square cross section. This cross-sectional shape would minimize the blending of the fibrils. However, it was observed that the fibrils close to the surface fibrils toward the top of the channel seemed to show less orientation than those on the bottom. This was 
attributed to the fine screening becoming blocked and thus interfering with the vacuum suction. The sample, once made, had to be "dug" out of the recessed channel, which led to the samples being pulled apart.

Samples were attempted using a thin core yarn. The core yarn positioned in the middle of the channel interfered with sample making because the flow of dispersant resulted in disorientation of fibrils. A different approach was taken using a 32-end braided cotton thread sheath. The braid was prefabricated and placed onto wet-laid samples. These braided-sheath samples were judged impractical for two reasons. First, the cotton thread, chosen for its burn-out potential, created an overly bulky sheath, stiffening the assembly excessively. In the interlaced structure of the braid, there are two layers of yarns which alternately exchange top and bottom positions, thus there is twice as much thickness to the sheath as is needed. The interstices (spaces) between the yarns in the braided structure were also potential places for loss of fibrils. A sheath was required which would provide adequate strength and also provide a completely planar abrasive resistant shield. A film sheath was the solution.

Further changes were made in the wet-laying process after evaluating the samples made using the single channel aluminum FAB.

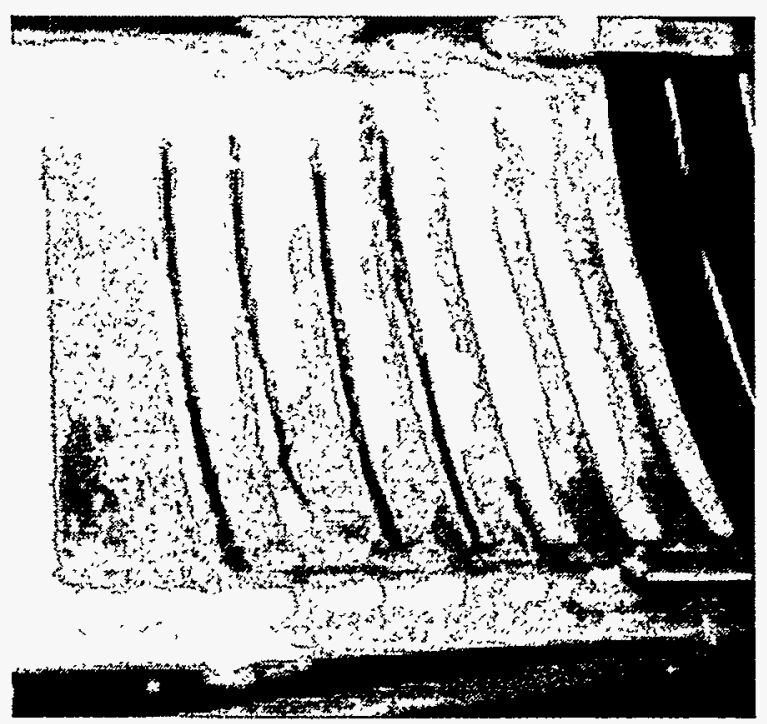

Figure 28. Multiple wet-laid assemblies on full screen. 
In order to meet the need for increased deliverables the SOW was changed to increase the amount of wet-laid product. In addition, a new scaled-up, multichannel, vacuum $F A B$ was required to produce longer samples. A commercially available FAB was constructed with a wooden-framed silk screen with polyester scrim screening. This screening was coarser than the screening used previously, thus clogging of the screen was eliminated. The frame was sealed to an aluminum sheet base with the tacky tape used frequently in vacuum bagging molding. Brass shims were added as support under the screening to prevent bowing of the screen under vacuum. Figure 28 shows that this bowing was not completely eliminated. Duct tape sealed off much of the screen and created individual vacuum tracks. Plastic sheeting was used to seal off all the tracks of the screen except for the track being used. The bowing of the screen created the need for a flexible set of runners to use as guides. These were made from a flexible rubber molding held in parallel position by a brace at either end. The flexible rubber conformed to the screen curvature when the vacuum would cause it to bow. These runners could be lifted off the screen to allow access to the sample. Once the runners were lifted off, a strip of paper toweling was laid over the sample while the vacuum was still on. The vacuum would pull the paper onto the damp sample causing adhesion. Once the vacuum was removed the paper towel was rolled off the screen with the fibril sample attached.

The same amount of dispersed fibrils was used to make the longer samples of the second vacuum box as was used in the first vacuum box. The dispersed fibrils $(250 \mathrm{ml}$ of a dispersant of 1.0 gram in $2500 \mathrm{ml}$ or 0.1 gram of fibrils per sample) were laid out over a longer and wider area to make a thinner, wider, tape-like sample. This was important to the bending behavior of the fibrils. This new geometry of the sample made it more flexible.

The access to the samples allowed visual evaluation before they were removed from the screen. This resulted in changing the method of wet-laying in order to improve the linear density consistency. The batch process of laying down the fibrils resulted in an excess of disoriented fibrils at either end of each sample, as the flow of dispersant is greater at the start and end of each channel. At specific intervals through the wet-laying process of each sample, the fibrils accumulating at either end would be removed from the sample and redispersed in the remaining measured dispersant. The 
added fibrils were not enough to disturb the orienting flow, but this practice ensured a more consistent sample.

\subsubsection{Integration}

Integrating the wet-laid assemblies requires a binder and a tape substrate with multiple properties. First, the binders and substrates must burn out cleanly and completely to allow the fibrils to be infiltrated in composite consolidation. Second, they must be sufficiently strong and flexible to allow the subsequent textile processing of the fibrils. Finally, they must be minimally voluminous to prevent excess void spaces upon burnout.

Integrating the wet-laid samples requires two binder qualities. A binder is required to give the wet-laid fibrils sufficient integrity to transfer them as a unit and to also provide adhesion once they dry. This was achieved by the PVA/water binder applied after the glycerin dispersion is rinsed off. Once dry, a tape substrate is laminated on either side of the fibrils, thus sealing them in and giving the assembly tensile strength, abrasion resistance, and cohesion during bending.

The tape substrate used was cellulose acetate film (office tape) because it adhered adequately to the fibrils. The tapes were found to be sufficiently strong and flexible, and they had been proven removable through burnout earlier in the program. It offers, however, only a preliminary solution for two reasons. First, the adhesion of the tape-to-tape lamination is insufficient to maintain the closed seam during severe bending. Second, at 2 mils thickness the film takes up too much volume in the entire assembly.

Film alternatives were sought but this remains an area for investigation. The burnout potential of polyethylene film, combined with its low-melting temperature, led to experimentation with heatsealing polyethylene film to form the sheath. Heat-sealing the polyethylene stiffened it too much to allow it to bend as readily as the cellulose acetate film and the strength of the heat-sealed bond was low.

The PVA used as the initial binder may prove problematic in this search because it is used as a release agent during molding operations. This quality may make it less likely to adhere to other 
binder agents and films. An alternative film may imply a n alternative binder agent.

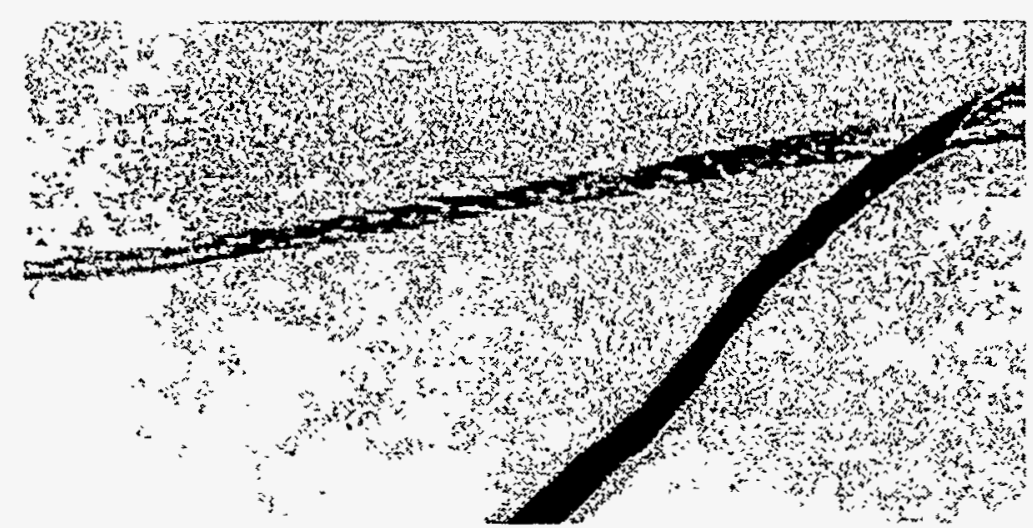

Figure 29. Wet-laid sample without tape substrate.

Figure 29 is a photograph of a wet-laid sample without the tape substrate. There is sufficient strength from the PVA binder to keep the fibrils integrated together, but the substrate is needed for sufficient subsequent processing strength.

\subsubsection{Characterization of Linear Fibril Assemblies}

The characterization of the linear fibril assemblies can be divided into two sections: the evaluation of the textile properties of the assembly and the evaluation of the properties relevant to composite fabrication and performance.

The textile evaluations of the linear assemblies quantify the properties which are relevant to the subsequent processing of the fibrils into different textile composite reinforcements. These properties include tensile strength, bending characteristics, and abrasion resistance. The composite reinforcement evaluations quantify the properties which make the fibril assemblies appropriate for the composite reinforcement end use and include fibril orientation and fibril volume fraction.

\section{Textile Evaluation}

The textile evaluations were conducted at the Philadelphia College of Textile and Science according to American Society for 
Testing and Materials (ASTM) Standards for textile evaluations. For safety reasons certain tests were not performed on the completed fibril assembly because the test procedures require testing to failure. Thus, a portion of the following tests were perforined solely on the auxiliary tape substrate material.

Tensile testing was performed on the auxiliary tape substrate before and after abrasion testing. Bend testing was performed on the completed fibril assembly in both single layer tape and double layer tape laminated versions.

\section{Tensile Testing}

Three films were tensile tested to failure according to ASTM testing standard 1682-64. Next, samples of the same films were abraded on a Tabor Abrasion Testing machine with Tabor abrasive wheel number CS-17 and a 500 gram weight on each rotary head. Samples were tensile tested again after 250 and 500 abrasive cycles to compare the loss in strength after abrasion. The results of these tests are tabulated in Table 2.

The first film tested was the cellulose acetate film used in the final deliverables. For comparison reasons, two other films were also tested. The first of these films was a 0.002 inch thick polyethylene film we had used for experiments in a heat sealing lamination technique. The second of the two alternative films tested was a 92 gauge polyester film.

Each of the three films exhibited sufficient strength for textile processing even after 250 and 500 abrasive rubs. This strength after abrasion is important because the film will be abraded to some degree during textile processing such as weaving, and it provides the sole tensile strength in the assembly. The film substrate also serves as a surface which protects the fibrils from abrasive wear. 
Table 2. Tensile Strength of Candidate Film Substrates in New and Abraded Samples.

\begin{tabular}{|c|c|c|c|c|}
\hline $\begin{array}{l}\text { Film Type } \\
\text { and Source }\end{array}$ & $\begin{array}{l}\text { Areal } \\
\text { Weight of } \\
\text { Film } \\
\text { (grams/ } \\
\text { square } \\
\text { meter) }\end{array}$ & $\begin{array}{l}\text { Tensile } \\
\text { Strength of } \\
\text { New Film } \\
\text { (grams/ } \\
\text { denier) }\end{array}$ & $\begin{array}{l}\text { Tensile } \\
\text { Strength of } \\
\text { Film after } \\
250 \\
\text { Abrasive } \\
\text { Cycles } \\
\text { (grams/ } \\
\text { denier) }\end{array}$ & $\begin{array}{l}\text { Tensile } \\
\text { Strength of } \\
\text { Film after } \\
500 \\
\text { Abrasive } \\
\text { Cycles } \\
\text { (grams/ } \\
\text { denier) }\end{array}$ \\
\hline $\begin{array}{l}0.001 " \text { thick } \\
\text { cellulose } \\
\text { acetate film } \\
\text { Source: } 3 \mathrm{M}\end{array}$ & 71.0 & 0.49 & 0.36 & 0.36 \\
\hline $\begin{array}{l}0.002 " \\
\text { thick } \\
\text { poly- } \\
\text { ethylene } \\
\text { film } \\
\text { Source: } \\
\text { Sunbelt } \\
\text { Plastics }\end{array}$ & 51.95 & 0.24 & 0.19 & 0.15 \\
\hline $\begin{array}{l}92 \text { Gauge } \\
\text { Polyester } \\
\text { Film } \\
\text { Source: } \\
\text { Flagship } \\
\text { Converters, } \\
\text { Inc. }\end{array}$ & 34.34 & 1.14 & 1.03 & 0.82 \\
\hline
\end{tabular}

\section{Flexural Testing}

Single-layer and double-layer tape and fibril assemblies were tested for bending stiffness, using adapted ASTM test method 1388 64 "Standard Test Methods for Stiffness of Fabrics." In this test method, the value for flexural rigidity in units of milligramcentimeters is calculated from a formula utilizing as variables: a) the area of a sample, b) the weight of the sample, and c) the length of the sample which will allow it to bend to a standard angle when unsupported and cantilevered over the measuring area. Table 3 lists 
the flexural rigidity calculated under this method for four different types of samples.

Table 3. Flexural Rigidity Values for Four Sample Types'

\begin{tabular}{|c|c|}
\hline Sample Type & $\begin{array}{c}\text { Average Flexural Rigidity } \\
\text { milligram-centimeters }\end{array}$ \\
\hline $\begin{array}{c}\text { Single Layer Cellulose Acetate } \\
\text { Tape - No Fibrils }\end{array}$ & 105 \\
\hline $\begin{array}{c}\text { Single Layer Cellulose Acetate } \\
\text { Tape - With Fibrils }\end{array}$ & 277 \\
\hline $\begin{array}{c}\text { Double Layer Cellulose Acetate } \\
\text { Tape - No Fibrils }\end{array}$ & 2445 \\
\hline $\begin{array}{c}\text { Double Layer Cellulose Acetate } \\
\text { Tape - With Fibrils }\end{array}$ & 8914 \\
\hline
\end{tabular}

The numbers are useful in comparison with each other, showing the large jump in rigidity which occurs when a double layer of tape is used with the fibril samples. In more practical terms, it is noted that double-layer tape and fibril samples were successfully woven into a small sample. This sample making in which fibril and tape samples were woven (and thus made to bend around each in the woven structure) indicates that the bending rigidity is sufficiently low to allow successful fabrication of textile samples.

Composite Reinforcement Evaluation

Evaluations of the linear orientation and fibril volume fraction are all key to the final composite end use of the fibrils.

An evaluation of the linear orientation is important to understand and evaluate the test results when testing the final fibril reinforced composite. Linear orientation evaluation was performed in two ways. First, as the deliverables were produced, the samples were evaluated visually as either acceptably or unacceptably oriented. Unacceptable samples were redispersed and rewet-laid. Second, a sample of the wet-laid fibril assembly and a sample of the adhesive-tape yarn were also tested by a crystal diffractometry method, suggested by Dr. Wei-Heng Shih of Drexel University. 
Fibril volume fraction was evaluated by potting fibril samples in resin to stabilize before cutting and viewing the cross section under magnification for volume calculation.

\section{Fibril Orientation}

A typical, visually acceptable, oriented sample is shown in Figure 2 in the opening Executive Summary section of this report. Crystal diffractometry plots are shown in Figures 30 and 31 . A typical plot of a wet-laid sample is shown in Figure 30 , which shows a more highly oriented product than the typical plot of the nonoriented, adhesive-tape-method sample shown in Figure 31.

\section{Fibril Volume Fraction}

The fibril volume fraction of the wet-laid product without a tape substrate was evaluated to be approximately $5 \%$. When a single layer of the tape was applied to the wet-laid fibrils, the fibril volume fraction dropped to approximately $2.5 \%$. The tape laminated in two layers around the fibrils resulted in an assembly fibril volume fraction of approximately $1.7 \%$.

To assist in achieving a maximum fibril volume fraction in the composite, the deliverables consisted of wet-laid assemblies made with a single layer of tape. Carborundum reported to APD that the maximum fibril volume fraction they were able to achieve in the assembly lay-up was $5 \%$.
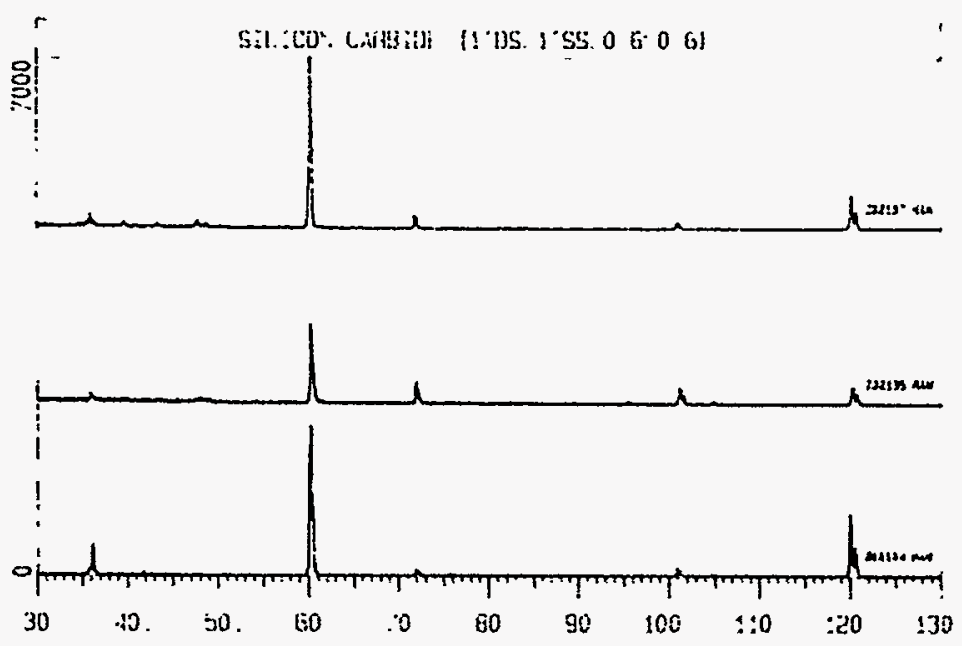

Figure 30. Crystal Diffractometry Plot of Wet-Laid Method Assembly. 

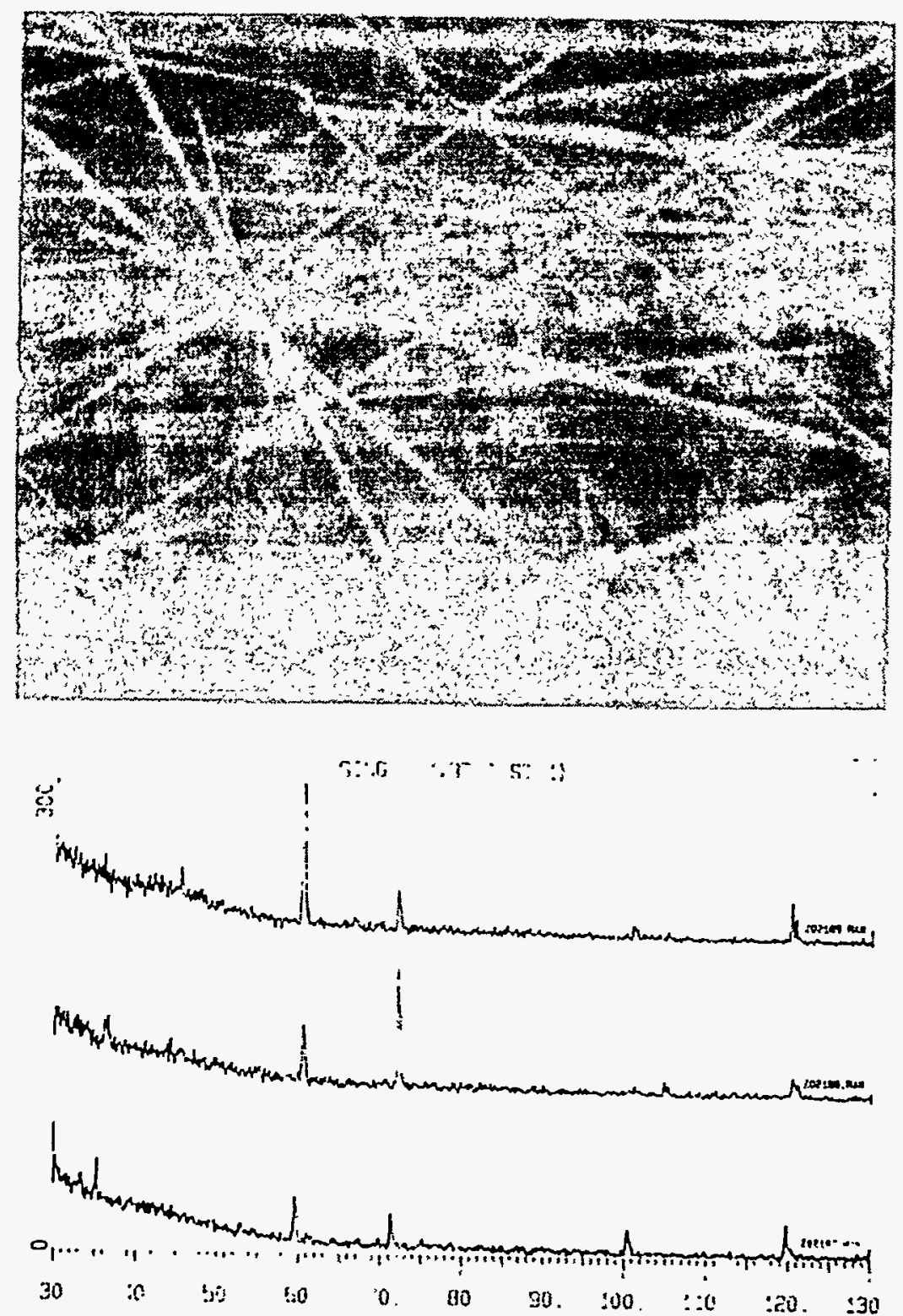

Figure 31. Photograph and crystal diffractometry plot of adhesivetape method assembly.

\section{Conclusions and Recommendations}

Three methods of processing $\mathrm{SiC}$ single-crystal fibrils into linear fibrous assemblies were evaluated. Traditional textile processing and the adhesive-tape method of working directly from a fibril growth plate were determined to be unacceptable for fibril yarn processing for the primary reason that neither method fulfilled 
a specific requirement for oriented fibrils. The single method which provided an oriented fibril array was the wet-laid process of aligning fibrils in the flow of a dispersant medium.

The wet-laid process provided a means to orient the fibrils, and preliminary methods were found to integrate them through a binder and auxiliary adhesive film sheath of materials which could later be burned out. The linear assembly deliverables were sufficiently oriented and integrated to fabricate into uniaxial lay-ups for composite trials for Carborundum contract Task 3.

The feasibility of the fluid-flow processing has been demonstrated, but additional work is required to evaluate the production potential of the method. A multiple-year Phase III proposal outlining additional work will be forwarded to Martin Marietta (Department of Energy) at the completion of the current contract. The following is a brief discussion of the major technical challenges to be addressed in the future work.

Within the next three-year time frame there are no plans by Carborundum to design and build a new reactor. All work for this proposal will depend on and utilize the fibrils produced in the existing reactor. Work with the fibrils and subsequent process development will therefore depend on the amount of fibrils that can be grown.

Carborundum engineers have explained the different ways in which the fibril growth reactor could deliver increased production of fibrils. Basically, these methods all require increasing throughput, with the maximum increase achieved by increasing the fibril growth surface area within the reactor. Increasing the surface area has the direct effect of decreasing the length of fibrils grown. As the fibril length decreases, the cost of growing the fibrils decreases. Modifications to the reactor to achieve a change of surface area would be costly both in downtime and fabrication, doubly so if a reversal had to be made due to premature execution of these modifications. Thus, additional work must focus on understanding the impact fibril length has on processing design and the simultaneous impact on linear assembly processing development, and how that impacts the end composite. The complexities of the fibril length issue begin to arise when one recognizes that the assembly processing will go through evolutionary changes from the current, inconsistent hand process to a semi-continuous process. 
Developing and testing such a semi-continuous design will require certain volumes of fibrils in different lengths. Conversely, characterizing the different fibril lengths in processing and end product will require a mechanized process to give consistent ${ }^{*}$ results for comparison. There is also the need to isolate the individual processes within the entire linear assembly process (i.e., dispersion ease. dispersion concentration, flow rate) to understand their interdependent nature, requiring additional volumes of fibrils. Much of the proposed work in engineer-hours resides in the tasks dedicated to developing a full understanding of the minimum fibril length required to make wet processing a continuous process.

\section{Flow Studies}

Because exploiting flow is key to the wet-laying process, future work must be dedicated to defining and utilizing all that is known about fibrils or fibers in fluid flow. A thorough literature search on these topics must be undertaken. A summary of the findings must be shared with all personnel involved with the program. The ramifications of the findings must be agreed upon as a departure point for subsequent experiments and machinery design.

\section{Dispersion}

As evidenced in Phase II, it is predicted that much of the challenge and effort of Phase III will be in the dispersion of fibrils. In Phase II the dispersing medium considered best at the initial feasibility stages was an $80 / 20$ glycerin/water mixture, the heavy viscosity of which both prevented "clumping" of the dispersed fibrils and promoted hand-controlled flow of the dispersion. In future work, however, it is predicted that a new dispersion medium and techniques will be sought as the wet-laid system is developed.

There are many challenges in dispersing the fibrils, both as an isolated step and as a major step within the complete linear assembly process. Considered as an isolated process, it is observed that fibril breakage and fibril waste has occurred when a large cluster of fibrils is placed in the dispersion medium. Because fibril growth is an expensive process which becomes greater as the length of fibril product increases, breakage and waste must be minimized. It is necessary to identify exactly how and when fibril entanglement occurs in order to address this issue. The processes of harvesting 
and leaching must be examined and modified to minimize entanglement.

\section{Subsequent Processing}

The consideration of dispersion within the entire assembly process raises many other issues. First, dispersion levels of fibrils in the dispersion medium have been very low to allow for easy movement and orientation of fibrils within the flow. Higher levels caused a sluggish, nonorienting flow. Using this dispersion level, we currently make over 100 repeated passes to lay down enough fibrils for a single yarn width. In a scaled-up continuous process, this would (in simplicity) imply: 1) 100 dispersion feed heads laying down, in sequence, on top of the same screen, or 2) "plying together" multiple screens. These unwieldy scenarios have an alternative in higher levels of dispersion which may be achieved by dispersing fibrils of shorter length. Because shorter fibrils are more economical to grow, the use of the shortest fibrils possible is ideal. Thus, studying the fibril length and the impact on the wet-laid process and product characterization is considered key to future work.

\section{Integration}

Integration is another challenge of future work which must be addressed throughout a continuous process. In the batch process currently used, a PVA solution is used as a binder after the fibrils are rinsed of trace amounts of glycerin. The binder gives the fibril array enough cohesiveness to be lifted from the screen before drying. Further support and tensile strength is provided by a tape substrate. An adhesive of some type is required because there is no other means by which to keep the rod-like fibrils in the desired axial orientation. However, in a scaleup situation it would be necessary to apply the binder earlier in the process. The wet-laid fibrils will require cohesion during continuous processing, and the level of this cohesion may be dependent on the fibril length. It may also indicate that the dispersion medium must have sufficient adhesive qualities to be activated. Another idea is to exploit the phenomenon of fibril flocking during $\mathrm{pH}$ changes for integration purposes.

\section{Textile Preforming}

The latter challenge of integration and adhesive choice has ramifications in designing for the end-product composite. The 
volume fraction of the finished yarn must allow for the inevitable volume fraction loss of textile preforming to achieve the desired volume fraction in the composite application. A consideration of this is the need to remove the binder from the fibril yarn before consolidation. Assuming this can be achieved through burnout, a major issue is whether the aforementioned designed volume fraction can then be controlled within the volume of the fixture once the volume of the binder is removed. These issues will begin to be addressed as the program tasks of textile preforming are implemented and results from tested ceramic coupons are analyzed.

\section{Machine Design}

A continuous process of any size will require machinery design, testing, modification, and retesting. The industrial technology closest to the requirements of this wet-laid process is paper making, but there are more differences than similarities. The relatively thick, water-based, cellulosic pulp from which paper is made is handled to encourage a random component fiber orientation. A paper web has inherent strength because of hydrogen bonding between the cellulosic fibers and subfibrils. Paper pulp itself is made by beating and other comparatively rough treatments of the component material. Many of the characteristics and processes of paper making will not be directly translatable to fibril processing. Specific considerations which must be included in a design for a semicontinuous fibril processing machine include the following:

1) Dispersion storage which can be continuously fed and agitated such that fibrils do not settle out and from which multiple feeds can be drawn.

2) Dispersion feed heads which do not clog with fibrils as flow is maintained.

3) A continuous moving wet-laying belt with vacuum drainage, which has some mechanism for being cleaned.

4) Wet waste storage tank, preferably separated into dispersion and adhesive materials, if potentially they can be recycled.

5) Adhesive application system with requisite curing area. 
6) An on-line substrate source and application.

A schematic drawing of the laboratory scale machine concept is shown below:

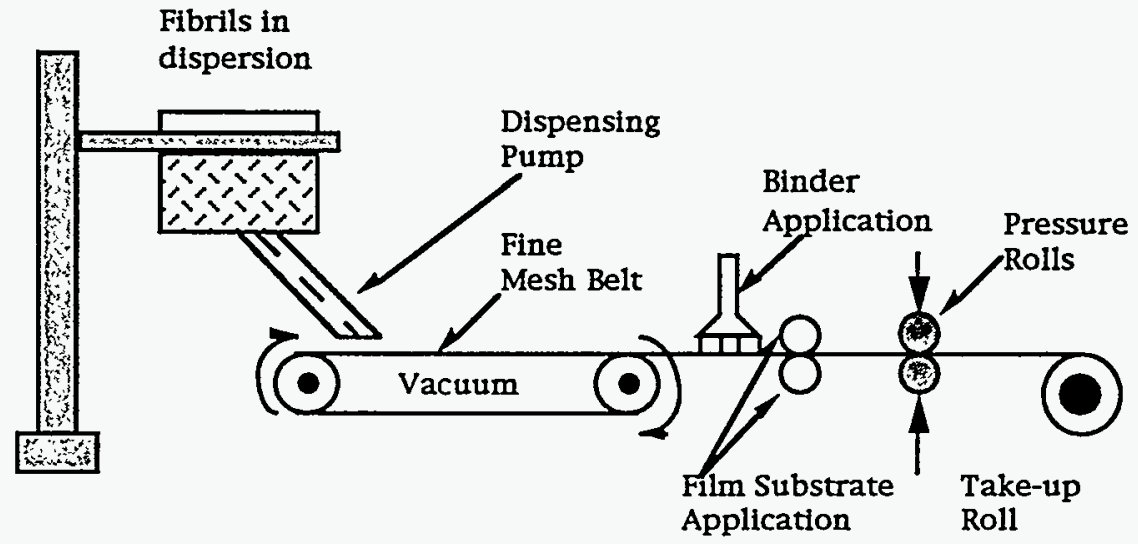

Figure 32. Schematic of the fluid-based, wet-laid system. 


\section{References}

Abbenheim, P. "Look! No Ring or Traveler." Textile Industries, May 1962, pp. 109-110.

American Society for Testing and Materials, 1989 Annual Book of ASTM Standards. Vols. 7.1, 7.2. Philadelphia: ASTM, 1989.

Anahara, M. "Integration of the Spinning Process." Textile Month, February 1990, pp. 23-25.

Berger, Hans. Asbestos Fundamentals, trans. by Ralph E. Oesper. New York: Chemical Publishing Company, Inc. 1963.

Booth, J. E. Principles of Textile Testing. London: Newnes Butterworths, 1968.

da Souza, L. "Break Spinning - A Breakthrough?" Man-Made Textiles, December 1965.

El-Homossani, M. M. "Producing Composite Yarns by Air-Vortex Spinning." Canadian Textile Journal, January, 1983, pp. 55-58.

General Applied Science Laboratories, Inc. "The Fluid Mechanics of Fibers: A Bibliography Technical Report." Technical Report No. 378, September 1963.

General Applied Science Laboratories, Inc. "Particle Trajectories in a Vortex-Sink Air Flow." Technical Report No. 532, May 1965.

Goswami, B. C.; J. G. Martindale, and F. L Scardino. Textile Yarns Technology. Structure, and Applications. New York: John Wiley \& Sons, 1977.

Gotzfried, Konrad. "Process and Apparatus for Spinning a Yarn." British Patent No. 825,776. December 1959.

Hamabata, S., M. Horiuchi, T. Samata and Y. Urano "Spinning Equipment Using Air-Flow." Journal of The Textile Machinery Society of Japan, Vol. 10, No. 4, 1994, pp.152-158. 
Hamby, Dame S., ed. The American Cotton Handbook - Volume 1. New York: John Wiley and Sons, 1965.

Hearle, J.W.S. and W.E. Morton,. Physical Properties of Textile Fibres. 2nd ed. London: William Heinemann, Ltd., 1975.

Hollar, William E., Jr. and Wendell H Mills. Engineering Scale Development of the Vapor-Liquid-Solid (VLS) Process for the Production of Silicon Carbide Fibrils. Report to Oak Ridge National Laboratory, Oak Ridge, TN, for Subcontract No. 86x-SD861C. September, 1993. Niagara Falls, NY: The Carborundum Company.

Kaswell, Ernest R. Wellington Sears Handbook of Industrial Textiles. Wellington Sears Company, 1963.

Kawabata, S. and M. Matsudaira. "Fiber Crimp Retention During Textile Processing and Its Effect on Fabric Quality." Objective Evaluation of Apparel Fabrics. October/November 1983, pp. 193-202.

Kimmel, L. B.; G. L. Louis, and H. L. Salaun.' "Ring Spun All Staple Core-Wrap Yarn - A Progress Report." Textile Research Journal, April 1989, pp. 244-246.

Kulter, H. and B. Wulfhorst. "Asbestos and Alternative Fibers." Chemiefasern/Textil-industrie, July/August 1990, pp. E70-E80.

Leifeld, F. "Technological Progress in Opening and Carding." Lenzinger Ber., February 1982, pp. 50-60.

Louis, G. L. and H.L. Salaun. "An Improved SRRC No-Twist Yarn System." Textile Research Journal, February, 1983, pp. 103-108.

Magnall, A. N.; F. Robers, and P. H. Taylor. "Asbestos Products," U. S. Patent 4,115,991, September 26, 1978.

McCreight, L.R.; H.W. Rauch, and W.H. Sutton. Ceramic Fibers and Fibrous Composite Materials. New York: Academic Press, 1968.

"New Safer, Cheaper, Lighter Asbestos Yarn." High Performance Textiles, April 1984, pp. 3-4. 
Raasch, H. "New Developments in Rotor Spinning." Textile

Technology International, 1991, pp. 119-122.

SAWTRI Sympsium on New Technologies For Cotton. Proceedings. July 1982.

Scardino, F. "Conversion of Boron Nitride Fiber Into Yarn on Gotzfried's Pneumatic Spinning Device." Unpublished report from Textile Research Institute to Fabric Research Laboratories, Inc. Princetun, NJ: June 14, 1966.

Scott, S. W. "Produce Asbestos Yarn Safely." Textile World, March 1981, pp. 69, 72.

Spinnbau, Bremen. "New Set of Cards For Processing Asbestos Substitute Fibers." Chemiefasern/Textil-industrie, October, 1986, pp. T91, E85.

Szaloki, Zoltan S. "High Speed Carding and Continuous Card Feeding." Vol. II of The Institute Series on Textile Processing. Charlottesville, VA: Institute of Textile Technology, 1977.

"Friction Spinning and Wrapping Pose the Main Challenge." Textile Month, July, 1983, pp. 28-32.

"Outline of Proposed Research on Multivariable Study of Fibers in Air Flow." Unpublished proposal from Textile Research Institute to Textile \& Apparel Technology Center, Institute for Applied Technology, U. S. Department of Commerce. Princeton, NJ: January 27, 1966. 


\section{Acknowledgments}

The authors gratefully acknowledge the following people for their invaluable assistance during the course of this program:

APD coworkers Richard Balonis, George Du, and Carol Taylor for technical, accounting, administrative support, and help in preparing this final report.

Consultant and Philadelphia College of Textiles \& Science Professor Frank Scardino for invaluable textile knowledge.

Mr. David J. Von Rohr of Drexel University for microscopy services.

Dr. Wei-Heng Shih of Drexel University for suggesting the crystal diffractometry method for evaluating fibril orientation.

Dr. Manal Shakar of Drexel University and University of Pennsylvania staff for crystal diffractometry services.

Professor Herb Barndt and Ms. Janet Brady of the Grundy Laboratory of Philadelphia College of Textiles \& Science for yarn characterization assistance.

Dr. William Hollar of Carborundum whose prompt responses to questions and comments made the program run smoothly.

Carborundum senior contract administrator Bonnie Mehls for patience and understanding in contract administration.

S. K. Lau, Chris Ebel, and Roger Ohnsorg of Carborundum for a smooth transition during personnel changes.

Nancy Cole and Ernie Long of DOE/ORNL for thoughtful questions during review presentations. 
Appendix 1. Alternatives to Traditional Textile Methods

Early in the program modifications and alternatives to the traditional textile methods were discussed at Cárborundum in Niagara Falls and at APD. The following tables list these modifications and alternatives.

Table A-1-1. Modifications to traditional textile processing.

\begin{tabular}{|c|c|c|}
\hline Modification & Advantages & Disadvantages \\
\hline $\begin{array}{c}\text { Fibrils vacuum } \\
\text { into sliver form } \\
\text { into directly }\end{array}$ & $\begin{array}{c}\text { 1) } \begin{array}{c}\text { Minimization of } \\
\text { fibril }\end{array} \\
\text { entanglement. }\end{array}$ & $\begin{array}{c}\text { 1) Catalyst balls } \\
\text { remain on fibrils. }\end{array}$ \\
$\begin{array}{c}\text { 2) Minimization of } \\
\text { fibril breakage } \\
\text { from } \\
\text { disentanglement. }\end{array}$ & $\begin{array}{c}\text { Sliver linear } \\
\text { plate linear } \\
\text { density. }\end{array}$ \\
$\begin{array}{c}\text { 3) Carborundum } \\
\text { trial-tested with } \\
\text { no discernible } \\
\text { success. }\end{array}$ \\
$\begin{array}{c}\text { Fibrils to be } \\
\text { sprayed with a } \\
\text { polymeric additive } \\
\text { to facilitate } \\
\text { spinning. }\end{array}$ & $\begin{array}{c}\text { 1) May cushion } \\
\text { forces of carding } \\
\text { and spinnning }\end{array}$ & $\begin{array}{c}\text { 1) May impede } \\
\text { disentanglement }\end{array}$ \\
\hline
\end{tabular}


Table A-1-2. Alternative fibril-to-yarn conversion methods.

\begin{tabular}{|c|c|c|}
\hline Method & Advantages & Disadvantages \\
\hline $\begin{array}{l}\text { Owens Corning } \\
\text { Fiberglass cardable } \\
\text { giass approach. }\end{array}$ & $\begin{array}{l}\text { Orientation potential } \\
\text { high. }\end{array}$ & Abrasive to fibrils. \\
\hline $\begin{array}{l}\text { Slurry extrusion } \\
\text { approach. }\end{array}$ & $\begin{array}{l}\text { Orientation potential } \\
\text { high. }\end{array}$ & $\begin{array}{l}\text { Low percentage of } \\
\text { fibrils. } \\
\text { TGA analysis of } \\
\text { sample supplied by } \\
\text { Carborundum } \\
\text { revealshigh } \\
\text { matrix/low fibril } \\
\text { content. } \\
\end{array}$ \\
\hline Wet-laid approach. & $\begin{array}{l}\text { Orientation potential } \\
\text { high. }\end{array}$ & $\begin{array}{l}\text { Dispersion of fibrils } \\
\text { may be difficult. }\end{array}$ \\
\hline Spun-laced approach. & $\begin{array}{l}\text { Non-twisting } \\
\text { entanglement. }\end{array}$ & $\begin{array}{l}\text { Low fibril orientation } \\
\text { potential. }\end{array}$ \\
\hline $\begin{array}{l}\text { Adhesive-bonding } \\
\text { approach. }\end{array}$ & $\begin{array}{l}\text { Orientation potential } \\
\text { high. }\end{array}$ & $\begin{array}{l}\text { Fibril catalyst balls } \\
\text { must be removed. }\end{array}$ \\
\hline $\begin{array}{l}\text { Adhesive-bonding } \\
\text { approach (Bobtex } \\
\text { approach). }\end{array}$ & $\begin{array}{l}\text { Strength from } \\
\text { adhesive. }\end{array}$ & Low fibril orientation. \\
\hline Flocking approach. & $\begin{array}{l}\text { Strength from } \\
\text { substrate. }\end{array}$ & $\begin{array}{l}\text { Low fibril orientation } \\
\text { potential. }\end{array}$ \\
\hline Core-spun approach. & Core strength. & High \%.core volume. \\
\hline $\begin{array}{l}\text { Water jet } \\
\text { entanglement. }\end{array}$ & $\begin{array}{l}\text { Entanglement } \\
\text { encourages } \\
\text { integration. }\end{array}$ & $\begin{array}{l}\text { Low fibril orientation } \\
\text { potential. }\end{array}$ \\
\hline $\begin{array}{l}\text { Spunbonded } \\
\text { approach. }\end{array}$ & $\begin{array}{l}\text { No twisting of fibrils } \\
\text { to integrate. } \\
\end{array}$ & Low fibril orientation. \\
\hline $\begin{array}{l}\text { Cross-wrapping } \\
\text { approach. }\end{array}$ & $\begin{array}{l}\text { No twisting of fibrils } \\
\text { to integrate. }\end{array}$ & $\begin{array}{l}\text { Cross-wrapping } \\
\text { material a high } \\
\text { volume } \% \text {. }\end{array}$ \\
\hline
\end{tabular}


Appendix 2.

Táble A-2. Comparison ơ SiC Fibrils and Textile Processed Asbestos.

\begin{tabular}{|l|c|c|}
\cline { 2 - 3 } \multicolumn{1}{c|}{} & $\begin{array}{c}\text { Carborundum SiC } \\
\text { Fibrils }\end{array}$ & $\begin{array}{c}\text { Textile Processed } \\
\text { Asbestos(1) }\end{array}$ \\
\hline Source & VLS crystal growth & $\begin{array}{c}\text { Crystalline rock } \\
\text { formation }\end{array}$ \\
\hline Fensity & $3.22 \mathrm{~g} / \mathrm{cm}^{3}$ & $2.55 \mathrm{~g} / \mathrm{cm}^{3}$ \\
\hline Fibril/Fiber Diameter & $3-10 \mathrm{microns}$ & $0.2-0.4 \mathrm{microns}(2)$ \\
\hline Tensile Strength & $\begin{array}{c}\text { Function of growth } \\
\text { process }(3)\end{array}$ & $\leq 2$ inches \\
\hline Modulus & $1 \mathrm{Msi}$ & $824 \mathrm{Ksi}$ \\
\hline Crimp & $70 \mathrm{Msi}$ & $45 \mathrm{Msi}$ \\
\hline
\end{tabular}

${ }^{1}$ Asbestos grades which are processed by textile machinery and techniques.

2 Acutal fibers - usually a coarser bundle of these is processed as a single fiber.

${ }^{3}$ Function of reactor parameters of component gas throughput, mixing, distance between growth substrates, etc., as per

Carborundum reporting. 
Appendix 3.

Table A-3. Comparison of SiC Fibrils and Chopped Nicalon Fiber.

\begin{tabular}{|l|c|c|}
\cline { 2 - 3 } \multicolumn{1}{c|}{} & SiC Fibrils & Chopped Nicalon \\
\hline Suurce & VLS Crystal Growth & Polymer Pyrolysis \\
\hline Density & $3.22 \mathrm{~g} / \mathrm{cm}^{3}$ & $2.74 \mathrm{~g} / \mathrm{cm}^{3}$ \\
\hline Fibril/Fiber Diameter & $3-10 \mathrm{microns}$ & $12-18 \mathrm{microns}$ \\
\hline Length & $\begin{array}{c}\text { Function of Growth } \\
\text { Process }\end{array}$ & $\begin{array}{c}\text { Filament Chopped to } \\
0.5 \mathrm{inch}\end{array}$ \\
\hline Tensile Strength & $1 \mathrm{msi}$ & $406 \mathrm{ksi}$ \\
\hline Modulus & $70 \mathrm{msi}$ & $39 \mathrm{msi}$ \\
\hline Crimp & None & None \\
\hline
\end{tabular}


Appendix 4. Fillet Clothing Carding Experimental Procedure

Carding Experiments With Hand Cards Equipped With

Sise Spacers Or Runners T0 Simulate Gauge Settings

1. Weigh out $\underline{x}$ grams of Nicalon and $(\underline{0.5-x)}$ grams of carrier fiber $(\mathrm{x}=0.05,0.10 .0 .15,0.20,0.25,0.30,0.35,0.40$, $0.45,0.50$ grams) to total 0.5 grams.

2. Combine Nicalon and carrier fiber samples and mix by hand plucking into spherical shape.

3. Place hand mixed sample ball onto one carding surface.

4. With a point-to-point carding direction, card the hand mixed sample for ten strokes in a vertical direction.

5. Photograph the carding surfaces to indicate fiber separation, blending, and orientation.

6. Photograph and weight the fiber fallout, if any, accumulated on surface under vertical carding action.

7. With a point-to-back carding direction, doff the carded sample from one carding surface and from the other carding surface to remove bulk of sample. Weigh the sample removed.

8. Photograph carding surfaces to indicate imbedded fibers/bundles, if any.

9. Manipulate the removed sample by straightening the doffed ball into a sliver form.

10. Photograph the sliver sample. 
Hand Drafting Experimental Procedure

1. Grasp/pinch one end of sliver sample and raise it up for a vertical suspension.

2. Photograph the sliver sample in the vertical suspended position.

3. Note whether or not the sliver sloughs apart or changes dimensions under its own weight.

4. Grasp/pinch both ends of sliver sample and gently extend or draft the sliver sample.

5. Photograph the drafted sample.

6. Note whether or not the sliver drafts uniformly with a cohesive drag or tends to pull apart in one spot. 
Hand Spinning Experimental Procedure

1. Grasp/pinch sliver at one end and compress/restrict sliver at about one inch from grasped end with fingers on other hand.

2. Extend and twist sliver at grasped end while restricting sliver with fingers on other hand to form a portion or segment of yarn/roving.

3. Take up the portion of hand twisted yarn/roving on index finger.

4. Repeat process (Steps \#1 to \#3).

5. Photograph the twisted yarn/rovins. 
Appendix 5. Alternatives to Fillet Card Clothing

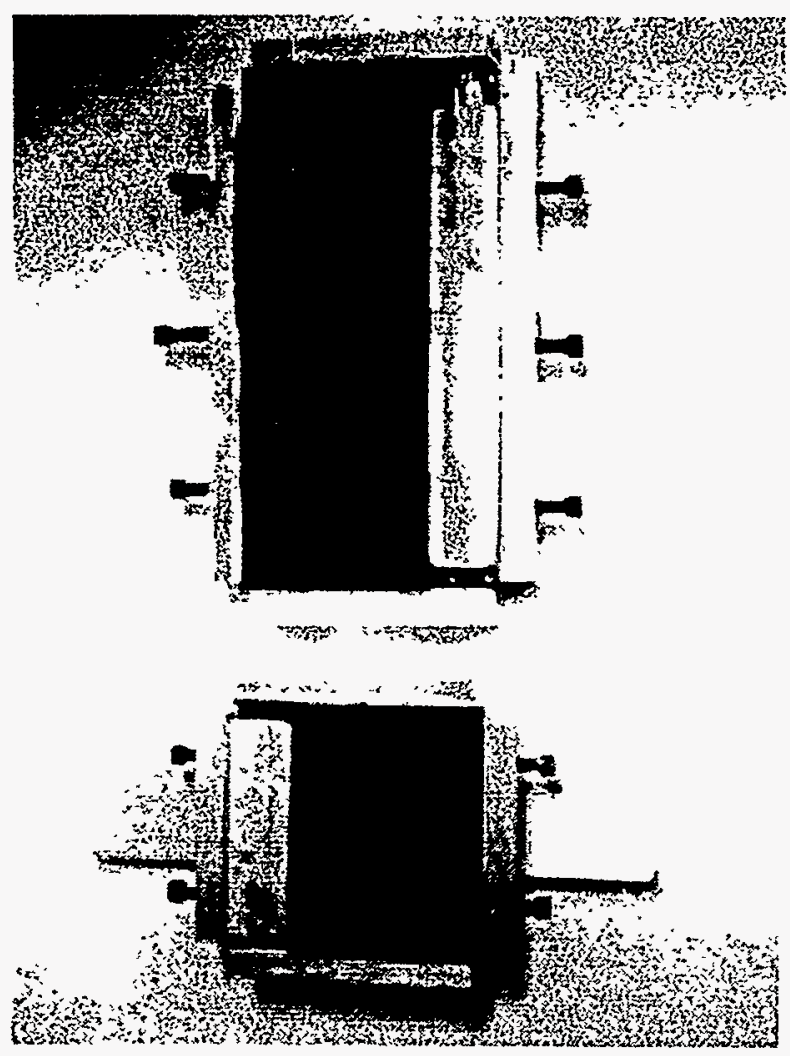

Figure A-5.1. Metallic clothing hand cards.

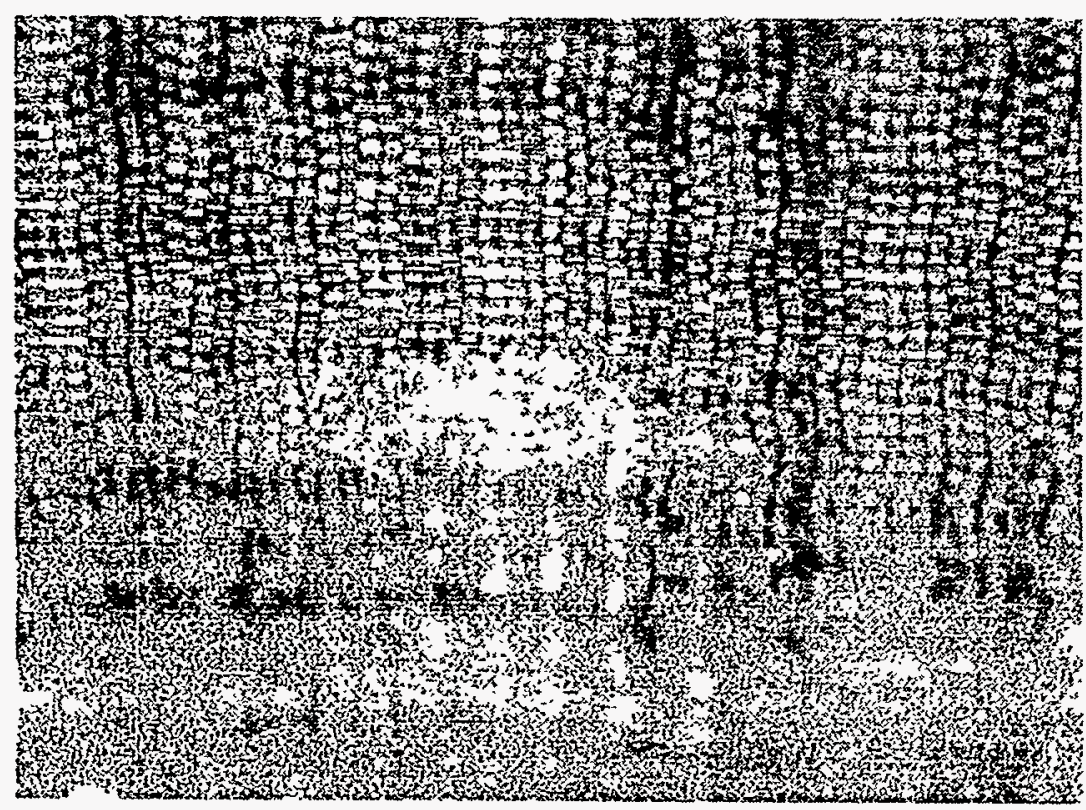

Figure A-5.2. Detail of fibrils on metallic card clothing. 


$6-\forall$

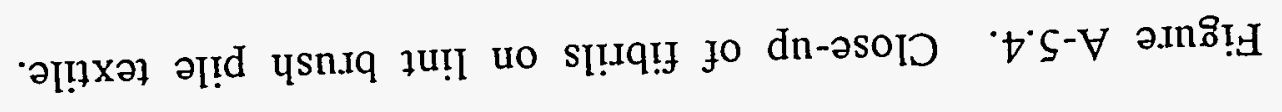

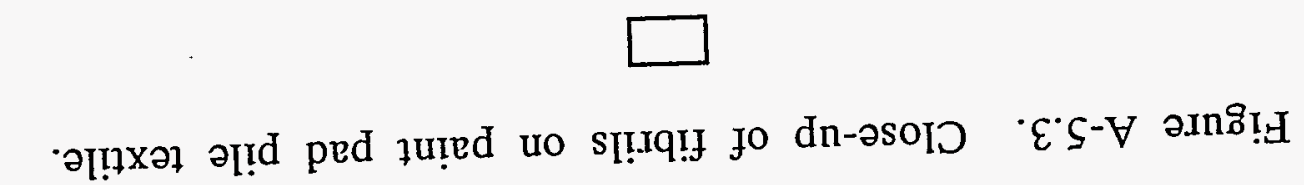

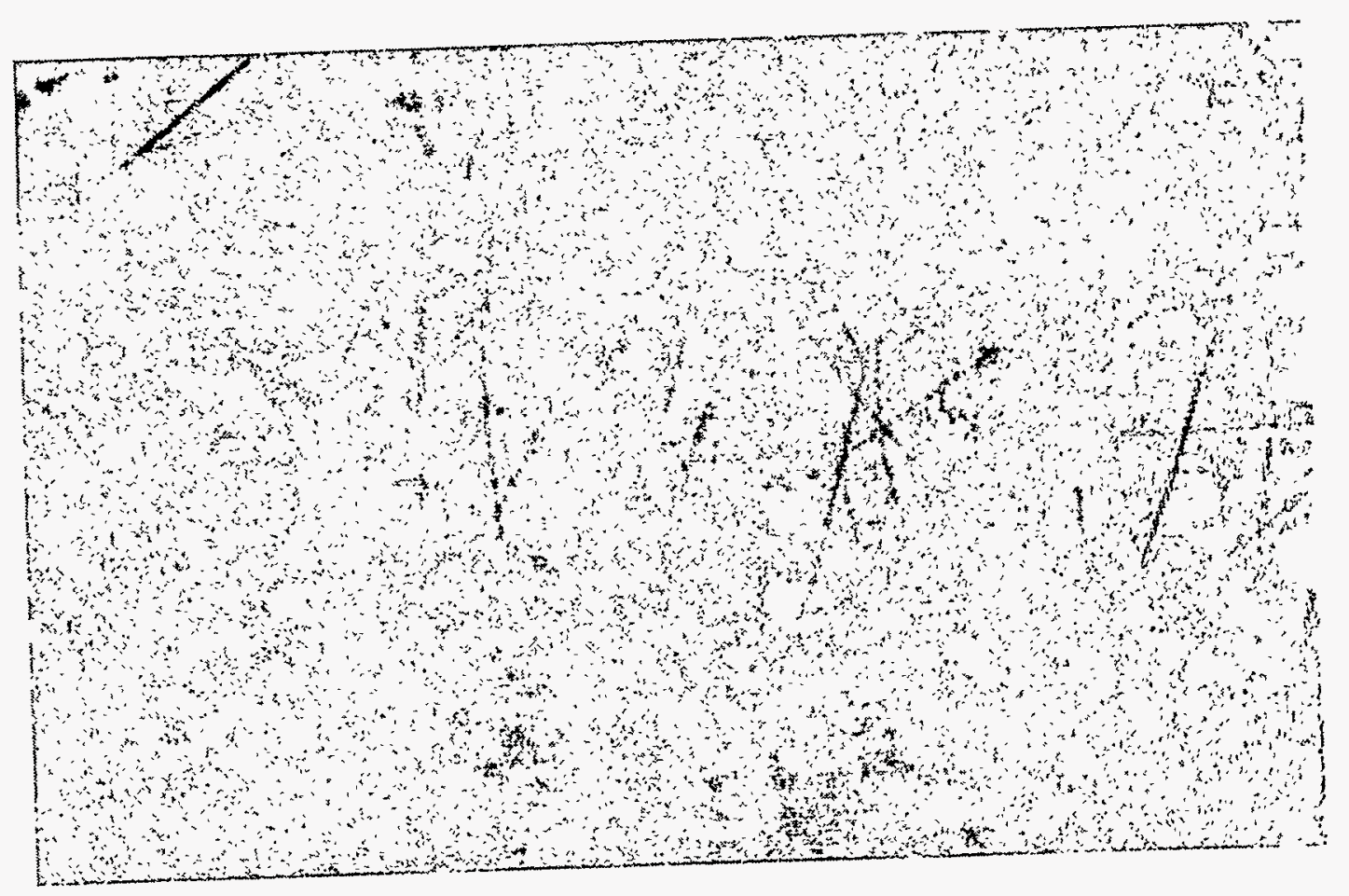


Appendix 6.

Weight Schematic Of Square Inch Plots Of Harvested Fibrils From 4 " X 10 " Center Of Fibril Growth Plate

\section{Plate \# 121 - Short}

\begin{tabular}{|c|c|c|c|}
\hline $.0122 \mathrm{gms}$ & $.0077 \mathrm{gms}$ & $.0092 \mathrm{gms}$ & $.0078 \mathrm{gms}$ \\
\hline $.0129 \mathrm{gms}$ & $.0092 \mathrm{gms}$ & $.0058 \mathrm{gms}$ & .0057 gms \\
\hline $.0132 \mathrm{gms}$ & $.0103 \mathrm{gms}$ & $.0071 \mathrm{gms}$ & $.0063 \mathrm{gms}$ \\
\hline $.0132 \mathrm{gms}$ & $.0083 \mathrm{gms}$ & $.0051 \mathrm{gms}$ & $.0073 \mathrm{gms}$ \\
\hline $.0139 \mathrm{gms}$ & $.0105 \mathrm{gms}$ & $.0065 \mathrm{gms}$ & $.0094 \mathrm{gms}$ \\
\hline .0183 & $.0106 \mathrm{gms}$ & $.0062 \mathrm{gms}$ & $.0097 \mathrm{gms}$ \\
\hline $.0189 \mathrm{gms}$ & $.0139 \mathrm{gms}$ & $.0078 \mathrm{gms}$ & $.0097 \mathrm{gms}$ \\
\hline $.0169 \mathrm{gms}$ & $.0140 \mathrm{gms}$ & $.0110 \mathrm{gms}$ & $.0081 \mathrm{gms}$ \\
\hline $.0155 \mathrm{gms}$ & $.0145 \mathrm{gms}$ & $.0106 \mathrm{gms}$ & .0075 \\
\hline $.0101 \mathrm{gms}$ & $.0091 \mathrm{gms}$ & $.0070 \mathrm{gms}$ & .0049 \\
\hline
\end{tabular}

Weight Schematic of Square Inch Plots of Harvested Fibrils from 4 " X 10 " Center of Fibril Growth Plate

Plate \# 1H- Modified Baseline

\begin{tabular}{|l|l|ll|ll|}
\hline $.0110 \mathrm{gms}$ & $.0064 \mathrm{gms}$ & $.0071 \mathrm{gms}$ & .0090 & $\mathrm{gms}$ \\
\hline $.0101 \mathrm{gms}$ & .0069 & $\mathrm{gms}$ & $.0092 \mathrm{gms}$ & .0109 & $\mathrm{gms}$ \\
\hline $.0107 \mathrm{gms}$ & $.0072 \mathrm{gms}$ & $.0100 \mathrm{gms}$ & .0155 & $\mathrm{gms}$ \\
\hline $.0097 \mathrm{gms}$ & $.0092 \mathrm{gms}$ & $.0155 \mathrm{gms}$ & .0123 & $\mathrm{gms}$ \\
\hline $.0108 \mathrm{gms}$ & $.0127 \mathrm{gms}$ & $.0125 \mathrm{gms}$ & $.0180 \mathrm{gms}$ \\
\hline $.0104 \mathrm{gms}$ & $.0163 \mathrm{gms}$ & $.0188 \mathrm{gms}$ & $.0194 \mathrm{gms}$ \\
\hline $.0199 \mathrm{gms}$ & $.0286 \mathrm{gms}$ & $.0146 \mathrm{gms}$ & $.0188 \mathrm{gms}$ \\
\hline $.0171 \mathrm{gms}$ & $.0251 \mathrm{gms}$ & $.0124 \mathrm{gms}$ & $.0146 \mathrm{gms}$ \\
\hline $.0151 \mathrm{gms}$ & $.0179 \mathrm{gms}$ & $.0149 \mathrm{gms}$ & $.0143 \mathrm{gms}$ \\
\hline $.0130 \mathrm{gms}$ & $.0151 \mathrm{gms}$ & $.0125 \mathrm{gms}$ & $.0107 \mathrm{gms}$ \\
\hline
\end{tabular}


Appendix 7. Photographs from Fibril Orienting Experiments

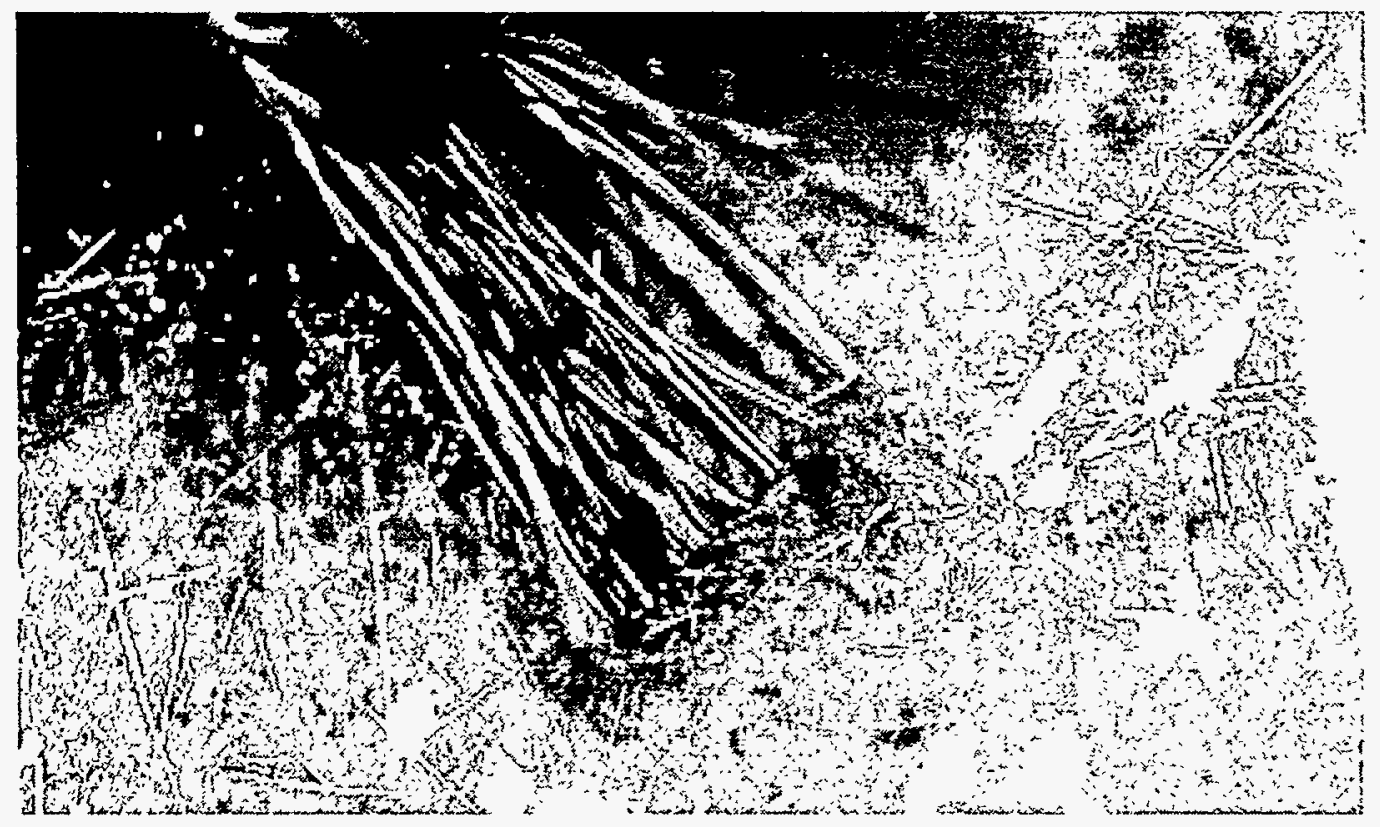

Figure A-7.1. Brush used on fibril growth.

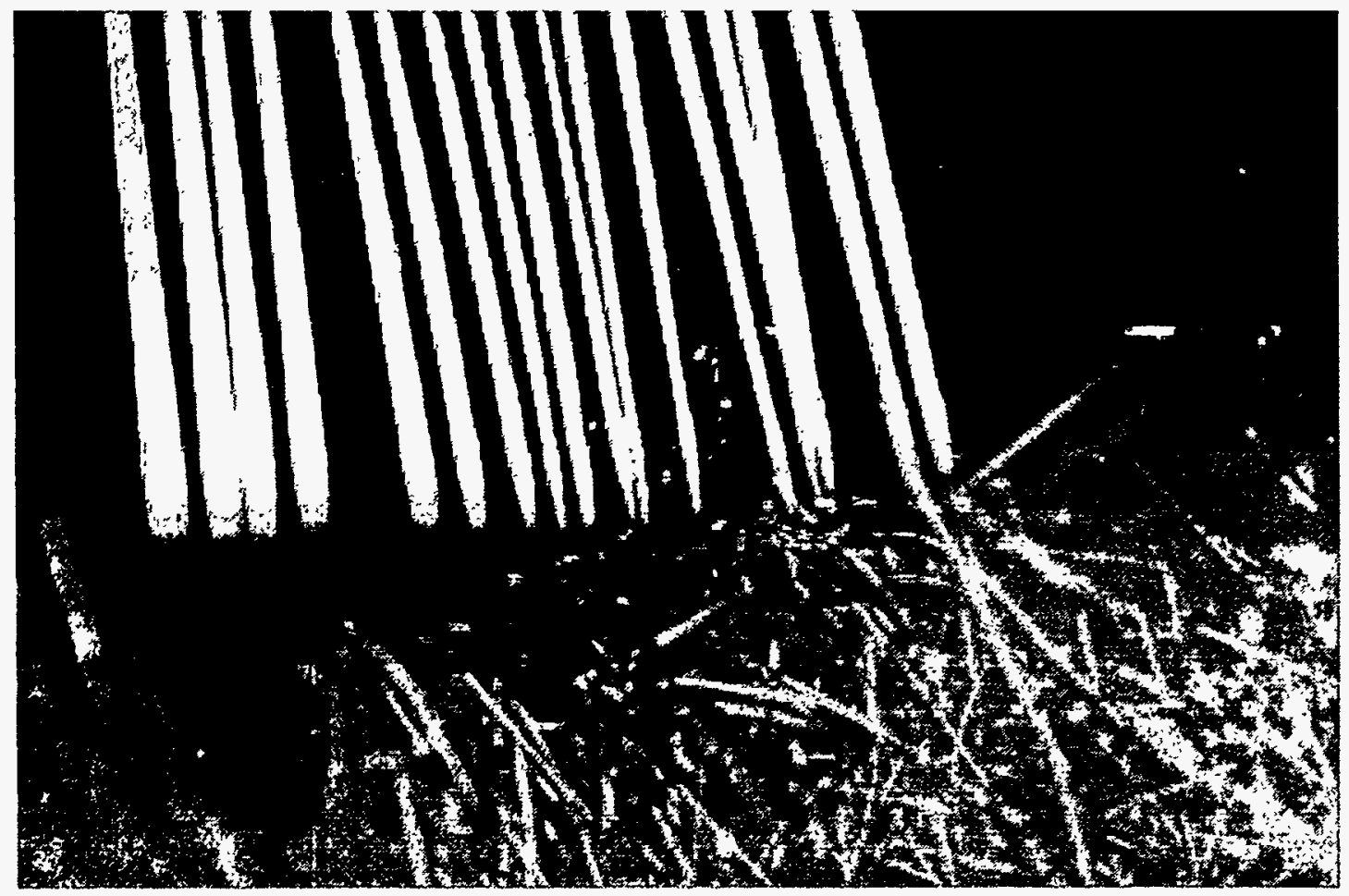

Figure A-7.2. Pin comb used on fibril growth. 


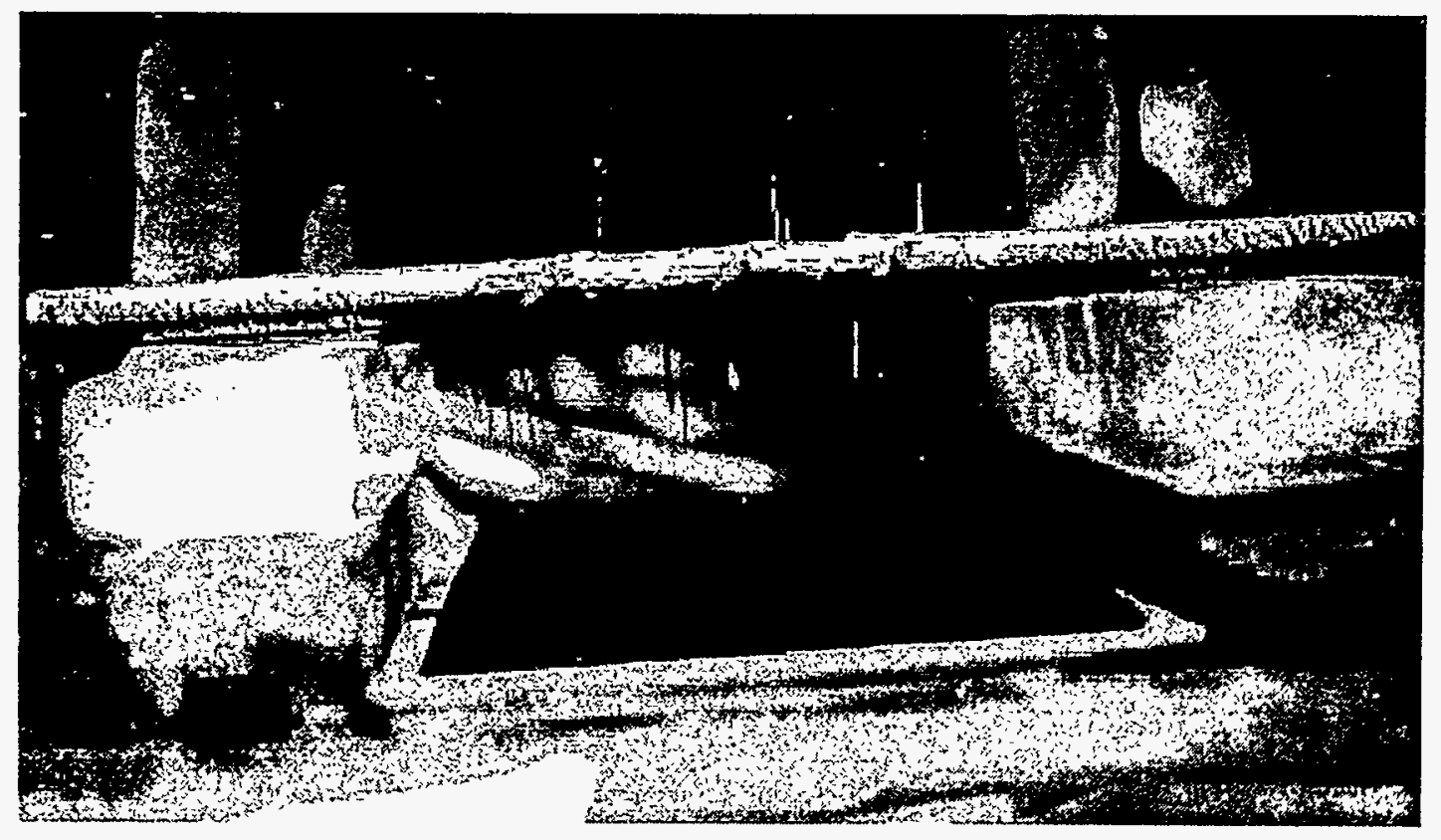

Figure A-7.3. Rotary comb.used on fibril growth.

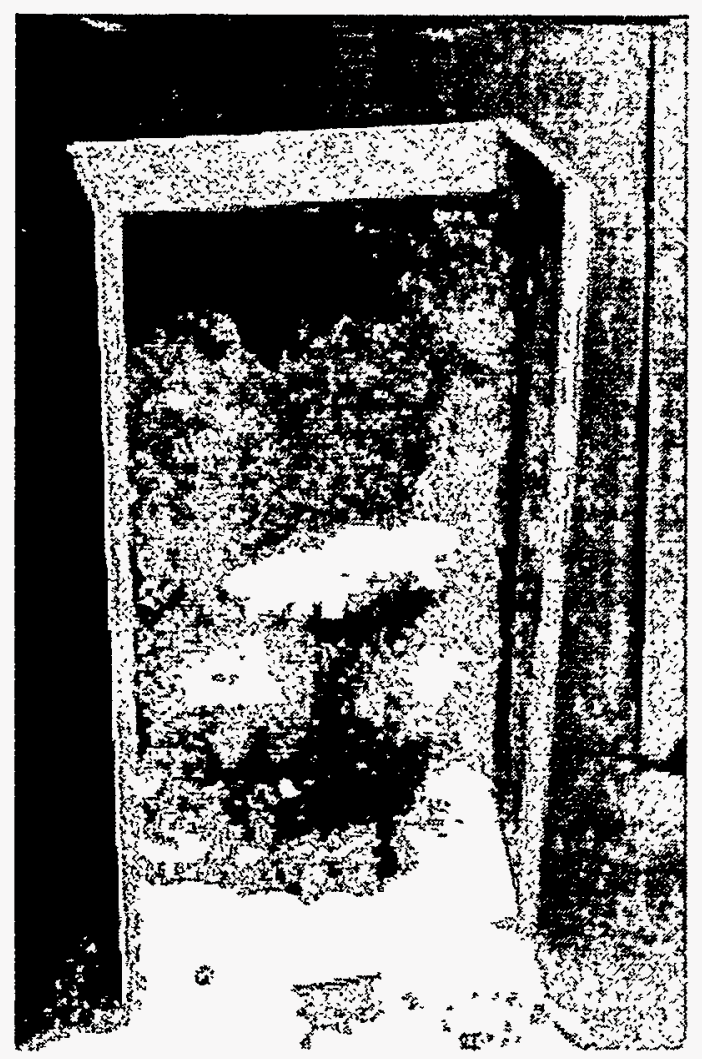

Figure A-7.4. Salt particles used on fibril plate. 


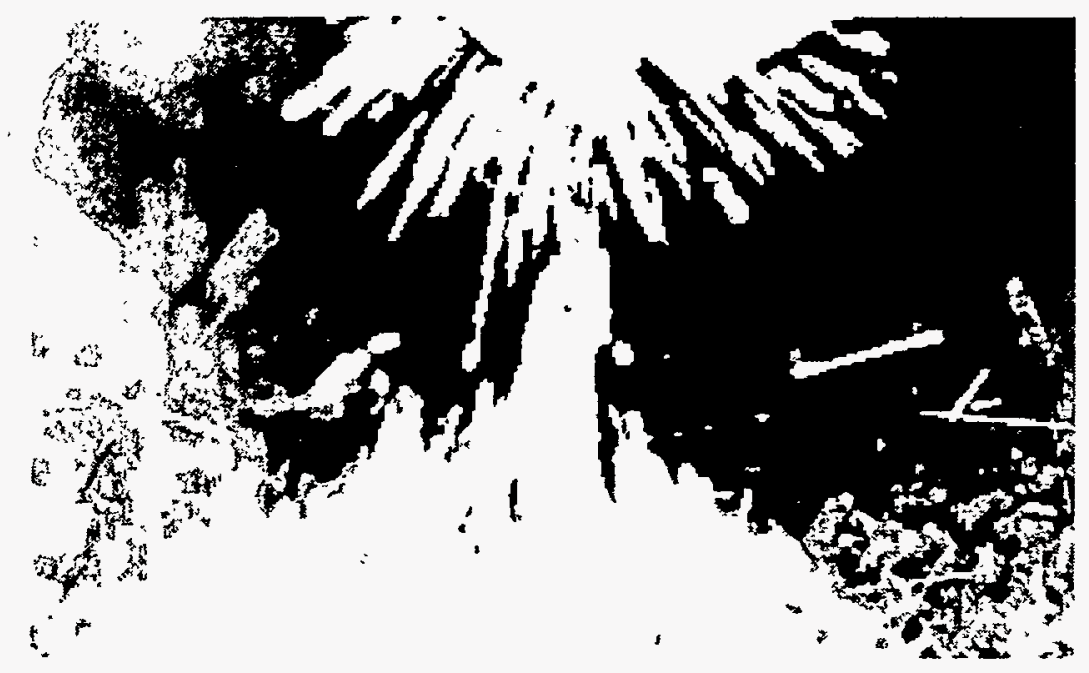

Figure A-7.5. Magnetic Powder used on fibril plates. 
Appendix 8. Clean Room and Safety Procedures

\section{A8.1 Entry}

1) A full-face respirator with HEPA filters is retrieved from storage and put on before entering booth.

2) Two pairs of disposable vinyl gloves are put on. Double gloving is used in case top layer tears and because it keeps 'Jottom glove set clean.

3) A full, new Tyvek suit with hood and feet is put on. The wrist suit/glove opening is taped with masking tape to seal.

4) A new plastic trash bag is mounted in booth for the easy disposal of wiping towels.

\section{A8.2 Work and Clean-Up}

1) As much as possible, work will occur within a confined area within the booth. For example, during work with the fibril plates, work progresses within cardboard walls such that the air currents do not disturb the work. This area will be thoroughly vacuumed with the HEPA vacuum after every use, and then wet-wiped with paper towels. Such a work area will be stored in plastic bags in the booth between uses.

2) All tools utilized during the course of working will be vacuumed, and then wet-wiped. During the work period vacuuming will occur as needed when tools are utilized and set aside. When leaving the confined work area described above, gloves will be vacuumed and wet-wiped to keep fibril particles from being deposited elsewhere via gloves.

3) All fibril sources, either in plate or loose, boxed form, will be closed and resealed before clean-up starts.

4) The greater area around the work area will be vacuumed first, then the rest of the booth will be vacuumed, starting from near the door and working backward toward the booth filters. Tabletops used 
will be vacuumed and wet-wiped. Wiping cloths will be disposable in a daily trash bag and this bag will be sealed and put in doubled plastic bags inside a covered trash can, and the tops of the bags closed inside the trash can between work periods.

5) Once a week, a more thorough vacuuming will take plate, including vertical surfaces, crevices, and other non-specific areas and surfaces.

\section{A8.3 Exit}

1) The respirator is vacuumed, and then wet-wiped.

2) Outside layer of gloves is removed and thrown away.

3) Tyvek suit is removed and thrown away.

4) Gloves are removed and thrown away. Trash bag is closed, deposited inside a doubled trash bag inside trash can, closed and covered.

5) Booth is departed, and only then is the respirator removed and stored, with the inside areas protected from dust with some kind of cover.

6) Doubled trash bag with a week's accumulation of daily bagged trash is sent out with the general facility trash once a week.

\section{A8.4 Additional Safety Suggestions}

During his visit to APD, Inc. the Carborundum Health, Safety, and Environment officer made helpful suggestions for improving the safety of the working environment. They are listed here for reference by future workers.

1) Keep the booth uncluttered so that the air flow is direct and uncluttered. Items stored in the booth can disrupt air currents and cause fibrils to lodge on surfaces instead of being vacuumed and filtered. 
2) Test the air currents within the booth with smoke generators to see if any furniture should be moved, etc. to promote direct airflow to the filters.

3) Work surfaces should be plastic laminate or a similar slick material to provide a smooth surface for removal of fibrils by wet-wiping.

4) Purchasing an additional local working hood under which to work with the fibrils within the overall vacuum environment is advised and would provide a magnitude level of increased cleanliness.

5) When removing Tyvek coveralls at the end of a work session, stand in directly in front of the air suction fans in order to remove any residual fibrils from the coveralls.

6) Store the respirator at all times in a plastic bag to keep clean.

7) Test connector panels of the booth and seal with silicone or other material. There are air leaks past the blowers but before the HEPA filters.

\section{Appendix 8.6 Fibril Safe Working Practices}

Toxicology studies have not been completely performed on the $\mathrm{SiC}$ fibrils, but the fine diameter of the $\mathrm{SiC}$ fibrils are similar to fibers which are known respiratory hazards, such as certain types of asbestos. Long-term health risks have dictated that safety precautions are required for any personnel working with the fibrils. Carborundum has indicated that in the future it intends to include the $\mathrm{SiC}$ fibrils as a product in their Stewardship Program, which develops and tracks safety practices used by customers of a product. Working together until this formal program is implemented, Carborundum and Advanced Product Development, Inc., have insured the safe handling of the fibrils by informed workers.

The first safety issue to be addressed was the need for a separate work area to prevent exposure to the fibrils by non-project personnel. An environmental control booth was installed before work with the fibrils began. Although not technically a clean room 
type facility, (it is intended for use as an isolation booth for industrial grinding operations), the booth is negatively pressurized and has had High Efficiency Particulate Air (HEPA) filters installed on the exhaust stack. Personal safety gear was purchased next. Fullface respirators with HEPA filters, Tyvek work coveralls with hood and boots, and vinyl gloves were obtained. Signs were posted on the booth entrance warning that respiratory protection was required hefore entering. APD developed guidelines for work practices for project personnel which were shared with co-workers ( Appendix 9).

Carborundum sent a Health, Safety, and Environment staff officer to observe working practices and to test the air for fibril exposure during a typical work day. He made suggestions for improving safe working conditions within the booth (Appendix 9). At this writing, the official safety evaluation report. of this visit is pending at Carborundum. 



\section{Appendix IIA}

\section{Economic Analysis}

Conservative Approach 



\section{Fibril Production Data for Different Furnace Configurations}

\begin{tabular}{|c|c|c|c|c|c|c|c|c|c|c|c|c|c|}
\hline $\begin{array}{l}\text { Reactor } \\
\text { Design }\end{array}$ & $\begin{array}{l}\text { Reactor } \\
\text { Size, in }\end{array}$ & $\begin{array}{l}\text { Plate } \\
\text { Size, in }\end{array}$ & $\begin{array}{l}\text { Plate } \\
\text { Thick., in }\end{array}$ & $\begin{array}{c}\text { Plate } \\
\text { Surface } \\
\text { Area, in2 }\end{array}$ & $\begin{array}{l}\text { Generator } \\
\text { Plate } \\
\text { Area, in2 }\end{array}$ & $\begin{array}{c}\text { Total } \\
\text { Surface } \\
\text { Area, ft2 }\end{array}$ & $\begin{array}{c}\# \\
\text { Spaces }\end{array}$ & $\begin{array}{l}\text { Distance } \\
\text { Between } \\
\text { Plates, in }\end{array}$ & $\begin{array}{l}\text { Distance } \\
\text { Between } \\
\text { Plates, cm }\end{array}$ & $\begin{array}{c}\text { Typical } \\
\text { Fibril } \\
\text { Length, } \mathrm{cm}\end{array}$ & $\begin{array}{c}\text { Specific } \\
\text { Growth Rate } \\
\mathrm{g} / \mathrm{ft} 2-\mathrm{h}\end{array}$ & $\begin{array}{l}\text { Product } \\
\text { q/Run }\end{array}$ & $\begin{array}{l}\text { Product } \\
\text { lb/Run }\end{array}$ \\
\hline 1 & $6 \times 6 \times 12$ & $6 \times 12$ & 0.125 & 288.0 & 212.4 & 3.475 & 4 & 1.344 & 3.414 & 1.500 & 0.500 & 8.687 & 0.01915 \\
\hline $2 a$ & $6 \times 6 \times 12$ & $6 \times 12$ & 0.125 & 288.0 & 212.4 & 3.475 & 4 & 1.344 & 3.414 & 1.500 & & & 0.02394 \\
\hline $2 a-1$ & & & & 288.0 & 212.4 & 3.475 & & & & 1.500 & 0.750 & 13.031 & 0.02873 \\
\hline $2 b$ & $6 \times 6 \times 12$ & $6 \times 12$ & 0.125 & 576.0 & 424.8 & 6.950 & 8 & 0.6406 & 1.627 & 0.750 & 0.500 & 8.687 & 0.01915 \\
\hline $2 b-1$ & & & & 576.0 & 424.8 & 6.950 & & & & 0.750 & 0.750 & 13.031 & 0.02873 \\
\hline $3 a$ & $12 \times 12 \times 42$ & $12 \times 12$ & 0.125 & 4032.0 & 2973.5 & 48.649 & 28 & 1.317 & 3.345 & 1.500 & 0.625 & 152.028 & 0.33516 \\
\hline $3 a-1$ & & & & 4032.0 & 2973.5 & 48.649 & & & & 1.500 & 0.750 & 182.434 & 0.40219 \\
\hline $3 b$ & $12 \times 12 \times 42$ & $12 \times 12$ & 0.125 & 8352.0 & 6159.3 & 100.773 & 58 & 0.6358 & 1.615 & 0.750 & 0.625 & 157.458 & 0.34713 \\
\hline $3 b-1$ & & & & 8352.0 & 6159.3 & 100.773 & & & & 0.750 & 0.750 & 188.949 & 0.41655 \\
\hline $4 a$ & $18 \times 18 \times 56$ & $18 \times 18$ & 0.188 & 11016.0 & 8123.9 & 132.916 & 34 & 1.344 & 3.414 & 1.500 & 0.625 & 415.362 & 0.91570 \\
\hline $4 a-1$ & & & & 11016.0 & 8123.9 & 132.916 & & & & 1.500 & 0.750 & 498.435 & 1.09884 \\
\hline $4 b$ & $18 \times 18 \times 56$ & $18 \times 18$ & 0.188 & 23328.0 & 17203.5 & 281.469 & 72 & 0.6346 & 1.612 & 0.750 & 0.625 & 439.795 & 0.96957 \\
\hline $4 b-1$ & & & & 23328.0 & 17203.5 & 281.469 & & & & 0.750 & 0.750 & 527.754 & 1.16348 \\
\hline
\end{tabular}




\section{Prototype Plant Throughput as a Function of Operating Factor and Yield Factor}

Fibril3n

(Reactor 3b)

\begin{tabular}{|l|r|r|r|r|}
\hline & \multicolumn{1}{|c|}{ Base } & \multicolumn{1}{|c|}{ Case I } & \multicolumn{1}{|c|}{ Case II } & \multicolumn{1}{|c|}{ Case III } \\
\hline Days/Year & 310 & 310 & 310 & 310 \\
Operating Factor & 0.85 & 0.85 & 0.90 & 0.90 \\
Cycles/Unit/Day & 1.655 & 1.655 & 1.655 & 1.655 \\
Cycles/Unit/Year & 436 & 436 & 462 & 462 \\
Yield/Cycle (kg) & 0.1575 & 0.1575 & 0.1575 & 0.1575 \\
& & & & \\
Annual Unit Capacity (kg) & 68.67 & 65.67 & 72.71 & 72.71 \\
Quality Yield Factor & 0.55 & 0.70 & 0.70 & 0.85 \\
Overall Efficiency Factor & 0.4675 & 0.5950 & 0.6300 & 0.7650 \\
Cost Factor Relative to Base & & 0.7857 & 0.7421 & 0.6111 \\
Annual Unit Yield (kg) & 37.77 & 48.07 & 50.90 & 661.81 \\
No. of Furnace Units & 6 & 6 & 6 & 6 \\
Plant Capacity (kg) & 226.62 & 288.43 & 305.39 & 370.84 \\
Plant Capacity (lb) & 499.70 & 635.98 & 673.40 & 817.69 \\
\hline
\end{tabular}

\section{Process Economics Depending on Throughputs for a Prototype Plant}

\begin{tabular}{|l|r|r|r|r|}
\hline & \multicolumn{1}{|c|}{ Base } & \multicolumn{1}{|c|}{ Case I } & Case II & \multicolumn{1}{|c|}{ Case III } \\
\hline Throughput $(\mathrm{lb} / \mathrm{y})$ & 499.70 & 635.98 & 673.40 & 817.69 \\
Capital Cost $(\$ 000)$ & 5355 & 5355 & 5355 & 5355 \\
Annual \$/lb & 10716 & 8420 & 7952 & 6549 \\
& & & & \\
Unit Operating Cost $(\$ / \mathrm{lb})$ & 79.56 & & & \\
$\quad$ Raw Materials & 8.16 & & & \\
Utilities & 570.43 & & & \\
$\quad$ Electricity & 1664.67 & & & \\
$\quad$ Process Gases & 604.96 & & & \\
Labor & 874.12 & & & \\
$\quad$ Maintenance & & & & \\
Indirect Charges & 3801.90 & 2987.21 & 2821.25 & 2323.38 \\
Total & & & & \\
\hline
\end{tabular}




\section{Prototype Plant Throughput as a Function of Operating Factor and Yield Factor}

Fbri3n1

(Reactor 3b-1)

\begin{tabular}{|c|c|c|c|c|}
\hline & Base & Case I & Case II & Case III \\
\hline Days/Year & 258 & 258 & 258 & 258 \\
\hline Operating Factor & 0.85 & 0.85 & 0.90 & 0.90 \\
\hline Cycles/Unit/Day & 1.655 & 1.655 & 1.655 & 1.655 \\
\hline Cycles/Unit/Year & 363 & 363 & 384 & 384 \\
\hline Yield/Cycle (kg) & 0.1889 & 0.1889 & 0.1889 & 0.1889 \\
\hline Annual Unit Capacity (kg) & 68.58 & 68.58 & 72.62 & 72.62 \\
\hline Quality Yield Factor & $0.55 !$ & 0.70 & 0.70 & 0.85 \\
\hline Overall Efficiency Factor & $0.4675 \mid$ & 0.5950 & 0.63001 & 0.7650 \\
\hline Cost Factor Relative to Base & & $0.7857:$ & 0.7421 & 0.6111 \\
\hline Annual Unit Yield (kg) & 37.72 & 48.011 & 50.831 & 61.73 \\
\hline No. of Furnace Units & 6 & 6 & $6 !$ & 6 \\
\hline Plant Capacity (kg) & 226.33 & 288.06 & 305.00 & 370.36 \\
\hline Plant Capacity (lb) & 499.06 & 635.16 & 672.52 & 816.64 \\
\hline
\end{tabular}

\section{Process Economics Depending on Throughputs for a Prototype Plant}

\begin{tabular}{|l|r|r|r|r|}
\hline & \multicolumn{1}{|c|}{ Base } & Case I & Case II & \multicolumn{1}{|c|}{ Case III } \\
\hline Throughput (lb/y) & 499.06 & 635.16 & 672.52 & 816.64 \\
Capital Cost (\$000) & 5355 & 5355 & 5355 & 5355 \\
Annual \$/lb & 10730 & 8431 & 7963 & 6557 \\
& & & & \\
Unit Operating Cost (\$/lb) & 72.04 & & & \\
$\quad$ Raw Materials & 6.80 & & & \\
Utilities & 475.53 & & & \\
$\quad$ Electricity & 1387.20 & & & \\
$\quad$ Process Gases & 605.74 & & & \\
Labor & 875.25 & & & \\
Maintenance & & & & \\
Indirect Charges & 3422.56 & 2689.15 & 2539.76 & \\
Total & & & & \\
\hline
\end{tabular}




\section{Semiworks Plant Throughput as a Function of Operating Factor and Yield Factor}

Fibril5n

(Reactor 4b)

\begin{tabular}{|c|c|c|c|c|c|}
\hline & & Base & Case I & Case II ; & Case III \\
\hline Days/Year & & $317^{\prime}$ & 317 & $317^{\prime}$ & 317 \\
\hline Operating Factor & & 0.85 & 0.85 & 0.90 & 0.90 \\
\hline Cycles/Unit/Day & 1 & $1.655^{\circ}:$ & 1.655 & 1.655 & 1.655 \\
\hline Cycles/U nit/Year & & $446^{\prime}$ & 446 & $472 !$ & 472 \\
\hline Yield/Cycle (kg) & ' & $0.4398 !$ & $0.4398 !$ & 0.4398 & 0.4398 \\
\hline Annual Unit Capacity (kg) & & 196.14 & 196.14 & 207.681 & 207.68 \\
\hline Quality Yield Factor & & 0.55 & 0.70 & 0.70 & 0.85 \\
\hline Overall Efficiency Factor & & 0.4675 & 05950 & 0.6300 & 0.7650 \\
\hline Cost Factor Relative to Base & & & 0.7857 & 0.7421 & 0.6111 \\
\hline Annual Unit Yield (kg) & & 107.88. & 137.30 & 145.38 ' & 176.53 \\
\hline No. of Furnace Units & & 42 & 42 & $42^{\circ}$ & 42 \\
\hline Plant Capacity (kg) & $\vdots$ & 4530.9 & $5766.6 !$ & 6105.8 & 7414.2 \\
\hline Plant Capacity (lb) & 1 & 9990.6 & 12715.3: & 13463.3 & 16348.3 \\
\hline
\end{tabular}

\section{Process Economics Depending on Throughputs for a Semiworks Plant}

\begin{tabular}{|c|c|c|c|c|c|}
\hline & 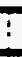 & Base & Case I & Case II & Case III \\
\hline Throughput (lb/y) & 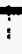 & 9990.6 & 12715.3 & 13463.3 & 16348.3 \\
\hline Capital Cost $(\$ 000)$ & ! & $53598 !$ & 53598 & 53598 & 53598 \\
\hline Annual $\$ / \mathrm{lb}$ & & $5365^{\circ}$ & 4215 & $3981 !$ & 3279 \\
\hline Unit Operating Cost $(\$ / \mathrm{lb})$ & & & & ! & \\
\hline Raw Materials & & 81.69 & & $\vdots$ & \\
\hline Utilities & & & & & \\
\hline Electricity & . & 5.70 & & & \\
\hline Process Gases & & 306.46 & & & \\
\hline Labor & & 398.92 & & & \\
\hline Maintenance & & 261.04 & & & \\
\hline Indirect Charges & & 382.11 & & & \\
\hline Total & & 1435.92 & 1128.22 & 1065.54 & 877.51 \\
\hline
\end{tabular}




\section{Semiworks Plant Throughput as a Function of Operating Factor and Yield Factor}

(Reactor 4b)

Fibril5n

\begin{tabular}{|l|r|r|r|r|}
\hline & \multicolumn{1}{|c|}{ Base } & \multicolumn{1}{|c|}{ Case I } & \multicolumn{1}{|c|}{ Case II } & \multicolumn{1}{|c|}{ Case III } \\
\hline Days/Year & 317 & 317 & 317 & 317 \\
Operating Factor & 0.85 & 0.85 & 0.90 & 0.90 \\
Cycles/Unit/Day & 1.655 & 1.655 & 1.655 & 1.655 \\
Cycles/Unit/Year & 446 & 446 & 472 & 472 \\
Yield/Cycle (kg) & 0.4398 & 0.4398 & 0.4398 & 0.4398 \\
& & & & \\
Annual Unit Capacity (kg) & 196.14 & 196.14 & 207.68 & 207.68 \\
Quality Yield Factor & 0.55 & 0.70 & 0.70 & 0.85 \\
Overall Efficiency Factor & 0.4675 & 0.5950 & 0.6300 & 0.7650 \\
Cost Factor Relative to Base & & 0.7857 & 0.7421 & 0.6111 \\
Annual Unit Yield (kg) & 107.88 & 137.30 & 145.38 & 176.53 \\
No. of Furnace Units & 42 & 42 & 42 & 42 \\
Plant Capacity (kg) & 4530.9 & 5766.6 & 6105.8 & 7414.2 \\
Plant Capacity (lb) & 9990.6 & 12715.3 & 13463.3 & 16348.3 \\
\hline
\end{tabular}

\section{Process Economics Depending on Throughputs for a Semiworks Plant}

\begin{tabular}{|l|r|r|r|r|}
\hline & \multicolumn{1}{|c|}{ Base } & Case I & Case II & Case III \\
\hline Throughput (lb/y) & 9990.6 & 12715.3 & 13463.3 & 16348.3 \\
Capital Cost $(\$ 000)$ & 53598 & 53598 & 53598 & 53598 \\
Annual \$/lb & 5365 & 4215 & 3981 & 3279 \\
& & & & \\
Unit Operating Cost $(\$ / \mathrm{lb})$ & 81.69 & & & \\
$\quad$ Raw Materials & 5.70 & & & \\
$\quad$ Utilities & 306.46 & & & \\
$\quad$ Electricity & 398.92 & & & \\
$\quad$ Process Gases & 261.04 & & & \\
Labor & 382.11 & & & \\
$\quad$ Maintenance & 1435.92 & 1128.22 & 1065.54 & \\
Indirect Charges & & & \\
Total & & & & \\
\hline
\end{tabular}




\section{Prototype Plant Throughput as a Function of Operating Factor and Yield Factor}

Fbri3n1

(Reactor $3 b-1$ )

\begin{tabular}{|l|r|r|r|r|}
\hline & \multicolumn{1}{|c|}{ Base } & \multicolumn{1}{|c|}{ Case I } & \multicolumn{1}{|c|}{ Case II } & \multicolumn{1}{|c|}{ Case III } \\
\hline Days/Year & 258 & 258 & 258 & 258 \\
Operating Factor & 0.85 & 0.85 & 0.90 & 0.90 \\
Cycles/Unit/Day & 1.655 & 1.655 & 1.655 & 1.655 \\
Cycles/Unit/Year & 363 & 363 & 384 & 384 \\
Yield/Cycle (kg) & 0.1889 & 0.1889 & 0.1889 & 0.1889 \\
& & & & \\
Annual Unit Capacity (kg) & 68.58 & 68.58 & 72.62 & 72.62 \\
Quality Yield Factor & 0.55 & 0.70 & 0.70 & 0.85 \\
Overall Efficiency Factor & 0.4675 & 0.5950 & 0.6300 & 0.7650 \\
Cost Factor Relative to Base & & 0.7857 & 0.7421 & 0.6111 \\
Annual Unit Yield (kg) & 37.72 & 48.01 & 50.83 & 61.73 \\
No. of Furnace Units & 6 & 6 & 6 & 6 \\
Plant Capacity (kg) & 226.33 & 288.06 & 305.00 & 370.36 \\
Plant Capacity (lb) & 499.06 & 635.16 & 672.52 & 816.64 \\
\hline
\end{tabular}

\section{Process Economics Depending on Throughputs for a Prototype Plant}

\begin{tabular}{|l|r|r|r|r|}
\hline & \multicolumn{1}{|c|}{ Base } & \multicolumn{1}{|c|}{ Case I } & Case II & \multicolumn{1}{|c|}{ Case III } \\
\hline Throughput $(\mathrm{Ib} / \mathrm{y})$ & 499.06 & 635.16 & 672.52 & 816.64 \\
Capital Cost $(\$ 000)$ & 5355 & 5355 & 5355 & 5355 \\
Annual \$/lb & 10730 & 8431 & 7963 & 6557 \\
& & & & \\
Unit Operating Cost $(\$ / \mathrm{lb})$ & 72.04 & & & \\
$\quad$ Raw Materials & 6.80 & & & \\
$\quad$ Utilities & 475.53 & & & \\
$\quad$ Electricity & 1387.20 & & & \\
$\quad$ Process Gases & 605.74 & & & \\
$\quad$ Labor & 875.25 & & & \\
Maintenance & & & & \\
Indirect Charges & 3422.56 & 2689.15 & 2539.76 & 2091.56 \\
Total & & & & \\
\hline
\end{tabular}




\section{Semiworks Plant Throughput as a Function of Operating Factor and Yield Factor}

Fbrilnn1

(Reactor $4 b-1$ )

\begin{tabular}{|l|r|r|r|r|}
\hline & \multicolumn{1}{|c|}{ Base } & \multicolumn{1}{|c|}{ Case I } & \multicolumn{1}{|c|}{ Case II } & \multicolumn{1}{c|}{ Case III } \\
\hline Days/Year & 264 & 264 & 264 & 264 \\
Operating Factor & 0.85 & 0.85 & 0.90 & 0.90 \\
Cycles/Unit/Day & 1.655 & 1.655 & 1.655 & 1.655 \\
Cycles/Unit/Year & 371 & 371 & 393 & 393 \\
Yield/Cycle (kg) & 0.5278 & 0.5278 & 0.5278 & 0.5278 \\
& & & & \\
Annual Unit Capacity (kg) & 196.02 & 196.02 & 207.55 & 207.55 \\
Quality Yield Factor & 0.55 & 0.70 & 0.70 & 0.85 \\
Overall Efficiency Factor & 0.4675 & 0.5950 & 0.6300 & 0.7650 \\
Cost Factor Relative to Base & & 0.7857 & 0.7421 & 0.6111 \\
Annual Unit Yield (kg) & 107.81 & 137.21 & 145.28 & 176.42 \\
No. of Furnace Units & 42 & 42 & 42 & 42 \\
Plant Capacity (kg) & 4528.0 & 5763.0 & 6101.9 & 7409.5 \\
Plant Capacity (b) & 9984.3 & 12707.3 & 13454.8 & 16338.0 \\
\hline
\end{tabular}

\section{Process Economics Depending on Throughputs for a Semiworks Plant}

\begin{tabular}{|c|c|c|c|c|}
\hline & Base & Case 1 & Case II & Case III \\
\hline Throughput (lb/y) & 9984.3 & 12707.3 & 13454.8 & 16338.0 \\
\hline Capital Cost $(\$ 000)$ & 53598 & 53598 & 53598 & 53598 \\
\hline Annual $\$ / \mathrm{lb}$ & 5368 & 4218 & 3984 & 3281 \\
\hline Unit Operating Cost & & & & \\
\hline Raw Materials & 73.53 & & & \\
\hline Utilities & & & & \\
\hline Electricity & 4.73 & & & \\
\hline Process Gases & 254.47 & & & \\
\hline Labor & 331.63 & & & \\
\hline Maintenance & 260.58 & & & \\
\hline Indirect Charges & 381.44 & & & \\
\hline Total & 1306.38 & 1026.44 & 969.42 & 798.34 \\
\hline
\end{tabular}



Appendix IIB

Economic Analysis

Aggressive Approach 



\section{Fibril Production Data for Different Reactor Configurations}

(With Graphite Rods as Growth Substrates*)

\begin{tabular}{|c|c|c|c|c|c|c|c|c|c|c|c|c|c|}
\hline $\begin{array}{l}\text { Reactor } \\
\text { Design }\end{array}$ & $\begin{array}{l}\text { Reactor } \\
\text { Size, in }\end{array}$ & $\begin{array}{l}\text { Plate } \\
\text { Size, in }\end{array}$ & $\begin{array}{l}\text { Plate } \\
\text { Thick., in }\end{array}$ & $\begin{array}{c}\text { Plate } \\
\text { Surtace } \\
\text { Area, in2 }\end{array}$ & $\begin{array}{l}\text { Generator } \\
\text { Plate } \\
\text { Area, in2 }\end{array}$ & $\begin{array}{c}\text { Total } \\
\text { Surface } \\
\text { Area, } \mathrm{ft} 2\end{array}$ & $\begin{array}{c}\# \\
\text { Spaces }\end{array}$ & $\begin{array}{l}\text { Distance } \\
\text { Between } \\
\text { Plates, in }\end{array}$ & $\begin{array}{l}\text { Distance } \\
\text { Between } \\
\text { Plates, cm }\end{array}$ & $\begin{array}{c}\text { Typical } \\
\text { Fibril } \\
\text { Length, } \mathrm{cm}\end{array}$ & $\begin{array}{c}\text { Specific } \\
\text { Growth Rate } \\
\mathrm{g} / \mathrm{ft} 2-\mathrm{h}\end{array}$ & $\begin{array}{l}\text { Product } \\
\text { g/Run }\end{array}$ & $\begin{array}{l}\text { Product } \\
\text { lb/Run }\end{array}$ \\
\hline 1 & $6 \times 6 \times 12$ & $6 \times 12$ & 0.125 & 288.0 & 212.4 & 3.475 & 4 & 1.344 & 3.414 & 1.500 & 0.9593 & 10.000 & 0.02205 \\
\hline $2 a$ & & $6 \times 12$ & & 288.0 & 212.4 & 3.475 & 4 & 1.344 & 3.414 & 1.500 & 1.4389 & 15.000 & 0.03307 \\
\hline $2 b$ & $6 \times 6 \times 12$ & $6 \times 12$ & 0.125 & 576.0 & 424.8 & 6.950 & 8 & 0.6406 & 1.627 & 0.750 & 1.4389 & 30.000 & 0.06614 \\
\hline $2 c$ & $6 \times 6 \times 12$ & $6 \times 12$ & 0.125 & & & 14.000 & 4 & 1.344 & 3.414 & 0.200 & 1.4286 & 60.000 & 0.13228 \\
\hline 3c & $12 \times 12 \times 42$ & $12 \times 12$ & 0.125 & & & 113.621 & 28 & 1.317 & 3.345 & 0.200 & 1.4286 & 486.947 & 1.07352 \\
\hline $4 c$ & $18 \times 18 \times 56$ & $18 \times 18$ & 0.188 & & & 313.767 & 34 & 1.344 & 3.414 & 0.200 & 1.4286 & 1344.716 & 2.96454 \\
\hline
\end{tabular}

*Growth substrate rod sizes $0.5^{\prime \prime} \times 12^{\mathrm{A}}$ for $3 \mathrm{C}$ and $0.5^{4} \times 18^{\mathrm{p}}$ for $4 \mathrm{C}$ 


\section{Prototype Plant Throughput as a Function of Operating Factor and Yield Factor}

Fbrl3c1

(Reactor 3c)

\begin{tabular}{|l|r|r|r|r|}
\hline & \multicolumn{1}{|c|}{ Base } & \multicolumn{1}{|c|}{ Case I } & \multicolumn{1}{|c|}{ Case II } & \multicolumn{1}{|c|}{ Gase III } \\
\hline Days/Year & 249 & 249 & 249 & 249 \\
Operating Factor & 0.85 & 0.85 & 0.90 & 0.90 \\
Cycles/Unit/Day & 2.000 & 2.000 & 2.000 & 2.000 \\
Cycles/Unit/Year & 423 & 423 & 448 & 448 \\
Yield/Cycle (kg) & 0.4869 & 0.4869 & 0.4869 & 0.4869 \\
& & & & \\
Annual Unit Capacity (kg) & 206.12 & 206.12 & 218.25 & 218.25 \\
Quality Yield Factor & 0.55 & 0.70 & 0.70 & 0.85 \\
Overall Efficiency Factor & 0.4675 & 0.5950 & 0.6300 & 0.7650 \\
Cost Factor Relative to Base & & 0.7857 & 0.7421 & 0.6111 \\
Annual Unit Yield (kg) & 113.37 & 144.29 & 152.77 & 185.51 \\
No. of Furnace Units & 2 & 2 & 2 & 2 \\
Plant Capacity (kg) & 226.74 & 288.57 & 305.55 & 371.02 \\
Plant Capacity (b) & 499.96 & 636.31 & 673.74 & 818.11 \\
\hline
\end{tabular}

\section{Process Economics Depending on Throughputs for a Prototype Plant}

\begin{tabular}{|l|r|r|r|r|}
\hline & \multicolumn{1}{|c|}{ Base } & \multicolumn{1}{|c|}{ Case I } & \multicolumn{1}{|c|}{ Case II } & \multicolumn{1}{|c|}{ Case III } \\
\hline Throughput (lb/y) & 499.96 & 636.31 & 673.74 & 818.11 \\
Capital Cost $(\$ 000)$ & 2512 & 2512 & 2512 & 2512 \\
Annual $\$ / \mathrm{lb}$ & 5.024 & 3.948 & 3.728 & 3.070 \\
& & & & \\
Unit Operating Cost $(\$ / \mathrm{lb})$ & & & & \\
$\quad$ Raw Materials & 45.11 & & & \\
$\quad$ Utilities & 2.71 & & & \\
$\quad$ Electricity & 184.36 & & & \\
$\quad$ Process Gases & 1225.04 & & & \\
$\quad$ Labor & 257.30 & & & \\
$\quad$ Maintenance & 479.53 & & & \\
$\quad$ Indirect Charges & & & & \\
Total & 2194.05 & 1723.90 & 1628.12 & 1340.81 \\
\hline
\end{tabular}




\section{Semiworks Plant Throughput as a Function of Operating Factor and Yield Factor}

Fbrl5c1

(Reactor 4c)

\begin{tabular}{|l|r|r|r|r|}
\hline & \multicolumn{1}{|c|}{ Base } & \multicolumn{1}{|c|}{ Case I } & \multicolumn{1}{|c|}{ Case II } & \multicolumn{1}{|c|}{ Case III } \\
\hline Days/Year & 301 & 301 & 301 & 301 \\
Operating Fastor & 0.85 & 0.85 & 0.90 & 0.90 \\
Cycles/Unit/Day & 2.000 & 2.000 & 2.000 & 2.000 \\
Cycles/Unit/Year & 512 & 512 & 542 & 542 \\
Yield/Cycle (kg) & 1.3447 & 1.3447 & 1.3447 & 1.3447 \\
& & & & \\
Annual Unit Capacity (kg) & 688.09 & 688.09 & 728.57 & 728.57 \\
Quality Yield Factor & 0.55 & 0.70 & 0.70 & 0.85 \\
Overall Efficiency Factor & 0.4675 & 0.5950 & 0.6300 & 0.7650 \\
Cost Factor Relative to Base & & 0.7857 & 0.7421 & 0.6111 \\
Annual Unit Yield (kg) & 378.45 & 481.66 & 510.00 & 619.28 \\
No. of Furnace Units & 12 & 12 & 12 & 12 \\
Plant Capacity (kg) & 4541.4 & 5780.0 & 6120.0 & 7431.4 \\
Plant Capacity (lb) & 10013.8 & 12744.8 & 13494.5 & 16386.2 \\
\hline
\end{tabular}

\section{Process Economics Depending on Throughputs for a Semiworks Plant}

\begin{tabular}{|l|r|r|r|r|}
\hline & \multicolumn{1}{|c|}{ Base } & \multicolumn{1}{|c|}{ Case I } & \multicolumn{1}{|c|}{ Case II } & \multicolumn{1}{|c|}{ Case III } \\
\hline Throughput (lb/y) & 10013.8 & 12744.8 & 13494.5 & 16386.2 \\
Capital Cost $(\$ 000)$ & 17419 & 17419 & 17419 & 17419 \\
Annual \$/lb & 1739.5 & 1366.8 & 1290.8 & 1063.0 \\
Unit Operating Cost $(\$ / \mathrm{lb})$ & & & & \\
$\quad$ Raw Materials & 46.03 & & & \\
$\quad$ Utilities & 1.87 & & & \\
$\quad$ Electricity & 100.42 & & & \\
$\quad$ Process Gases & 183.76 & & & \\
Labor & 82.91 & & & \\
Maintenance & 133.80 & & & \\
$\quad$ Indirect Charges & & & & \\
Total & 548.79 & 431.19 & & \\
\hline
\end{tabular}




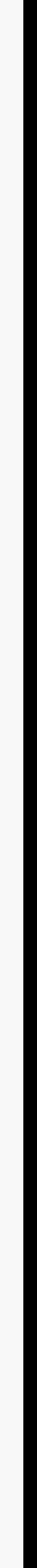




\section{Arnold}

British Coal Corporation

Coal Research Establishment

Stoke Orchard, Cheltenham

Glochester, England GL52 4RZ

Assistant Manager for Energy

Research \& Development

DOE

DGL Oak Ridge Operaiions

P.O. Box 2001

Oak Ridge, TN 37831

H. Atwell

Dow Corning Corporation

3901 S. Saginaw Road

Midland, MI 48686-0995

R. A. Bajura

DOE

Morgantown Energy Technology Center

P.O. Box 880

Morgantown, WV 26505

W. T. Bakker

Electric Power Research Institute

P.O. Box 10412

3412 Hillview Avenue

Palo Alto, CA 94303

A. L. Baldwin

DOE

Pittsburgh Energy Technology Center

P.O. Box 10940

Pittsburgh, PA 15236

C. P. Ballard

Allied Signal, Inc.

Ceramics Program

P.O. Box 1021

Morristown, NJ 07962-1021

R. C. Bedick

DOE

Morgantown Energy Technology Center

P.O. Box 880

Morgantown, WV 26505
C. Bower

British Coal Corporation

Coal Research Establishment

Stoke Orchard, Cheltenham

Glochester, England GL52 4RZ

R. Bradley

Oak Ridge National Laboratory

P.O. Box 2008

Oak Ridge, TN 37831

T. D. Burchell

Oak Ridge National Laboratory

P.O. Box 2008

Oak Ridge, TN 37831

R. J. Buss

Sandia National Laboratories

Department 6211, MS 0710

Albuquerque, NM 87185

G. A. Carlson

Sandia National Laboratories

Department 6211, MS 0710

Albuquerque, NM 87185

P. T. Carlson

Oak Ridge National Laboratory

P.O. Box 2008

Oak Ridge, TN 37831

J. P. Carr

DOE

Office of Fossil Energy

FE-72

19901 Germantown Road

Germantown, MD 20874-1290

Harry Cheung

Union Carbide Corporation

Linde Division

P.O. Box 44

175 East Park Drive

Tonawanda, NY 14151-0044 
W. J. Chmura

The Torrington Company

Advanced Technology Center

59 Field Street

Torrington, CT 06790

D. C. Cicero

DOE

Morgantown Energy Technology Center

P.O. Box 880

Morgantown, WV 26505

W. S. Coblenz

U.S. Department of Defense

Advanced Research Project Agency

3701 North Fairfax Drive

Arlington, VA 22203-1714

N. C. Cole

Oak Ridge National Laboratory

P.O. Box 2008

Oak Ridge, TN 37831

N. Corbin

The Norton Company

High Performance Ceramics Division

Goddard Road

Northborough, MA 01532-1545

T. B. Cox

AMA Research \& Development Center 5950 McIntyre Street

Golden, CO 80403

F. W. Crouse, Jr.

DOE

Morgantown Energy Technology Center

P.O. Box 880

Morgantown, WV 26505

W. A. Curtin

Virginia Polytechnic Institute \& State University

Department of Materials Engineering

Blackburg, VA 24601
J. B. Darby

DOE

Office of Basic Energy Sciences

Materials Sciences Division

ER-131

19901 Germantown Road

Germantown, MD 20874-1290

S. W. Dean

Air Products and Chemicals

P.O. Box 538

Allentown, PA 18105

L. W. R. Dicks

Shell Development Company

P.O. Box 1380

Houston, TX 77251-1380

DOE

Office of Scientific \& Technical Information

P.O. Box 62

Oak Ridge, TN 37831

For distribution by microfiche as shown in DOE/TIC-4500

Distribution Category: UC-114

(Coal Based Materials \& Components)

B. Draskovich

Allied Signal Aerospace

Garrett Engine Division

111 S. 34th Street

P.O. Box 5217

Phoenix, AR 85010-5217

J. DiCarlo

NASA-Lewis Research Center

21000 Brookpark Road

Cleveland, $\mathrm{OH} 44135$

J. J. Eberhardt

DOE

Office of Conservation and Renewable

Energy

CE-12 Forrestal Building

Washington, DC 20545 
W. A. Ellingson

Argonne National Laboratory

9700 S. Cass Avenue

Argonne, IL 60439

B. Bartlett

Pratt \& Whitney

P.O. Box 109600

West Palm Beach, FL 33410-9600

A. G. Evans

Division of Applied Sciences

Harvard University

311 Pierce Hall

Cambridge, MA 02138

S. G. Fishman

Office of Naval Research

Code 431, 800 N. Quincy Street

Arlington, VA 22217

F. Gac

Los Alamos National Laboratory

P.O. Box 1663

Los Alamos, NM 87545

M. Gold

Babcock \& Wilcox

Domestic Fossil Operations

20 South Van Buren Avenue

Baberton, OH 44023

R. E. Green, Jr.

The Johns Hopkins University

Materials Science \& Engineering

Maryland Hall

Baltimore, MD 21218

T. D. Gulden

GA Technologies, Inc.

P.O. Box 85608

San Diego, CA 92138

P. W. Heitman

General Motors Corporation

Allison Gas Turbine Division

P.O. Box 420, MS: W-5

Indianopolis, IN 46206-0420
S. P. Henslee

Argonne National Laboratory-West

P.O. Box 2528

Idaho Falls, ID 83403-2528

J. L. Hill

A.P. Green Refractories Company

Green Bivd.

Mexico, MO 65265

E. E. Hoffman

DOE

DOE Oak Ridge Operations

P.O. Box 2008

Building 4500N, MS 6269

Oak Ridge, TN 37831

N. T. Holcombe

DOE

Morgantown Energy Technology Center

P.O. Box 880

Morgantown, WV 26505

C. M. Huang

Tennessee Valley Authority

Energy Demonstration \& Technology

MR2N58A

Chattanooga, TN 37402-2801

W. J. Huber

LOE

Morgantown Energy Technology Center

P.O. Box 880

Morgantown, WV 26505

M. A. Janney

Oak Ridge National Laboratory

P.O. Box 2008

Oak Ridge, TN 37831
A. M. Johnson
G.E. Aircraft Engines
One Neumann Way, MD H85
Cincinnati, OH 45215-6301 
T. I. Johnson

Babcock \& Wilcox

1562 Beeson Street

Alliance, $\mathrm{OH} 44601$

R. R. Judkins

Oak Ridge National Laboratory

P.O. Box 2008

Oak Ridge, TN 37831

\section{A. Katz}

Wright Laboratory

Metals and Ceramics Division

WL/MLLM

Wright-Patterson AFB, OH 45433

J. D. Katz

Los Alamos National Laboratory

P.O. Box 1663

Los Alamos, NM 87545

D. W. Keefer

Idaho National Engineering Laboratory

P.O. Box 1625

Idaho Falls, ID 83415

D. J. Kenton

EC Technologies

3614 Highpoint Drive

San Antonio, TX 78217

P. Khandelwal (Speed Code W-5)

Allison Gas Turbine Division

P.O. Box 420

Indianapolis, IN 46206-0420

S. K. Lau (25 copies)

The Carborundum Company

Technology Division

P.O. Box 832

Niagara Falls, NY 14302

R. A. Lawson (8 copies)

Oak Ridge National Laboratory

P.O. Box 2008

Oak Ridge, TN 37831
M. A. Leitheiser

3M Company

Ceramic Materials Department

201-4N-01 3M Center

St. Paul, MN 55144

G. D. Linsey

Pratt \& Whitney

400 Main Street

East Hartford, CT 06108

E. L. Long, Jr.

Oak Ridge National Laboratory

P.O. Box 2008

Oak Ridge, TN 37831

R. B. Loop

DOE

Idaho Operations Office

P.O. Box 1625

Idaho Falls, ID 83415

S. G. Malghan

National Institute of Standards \& Technology

U. S. Dept. of Commerce

Building 220, Room A215

Gaithersburg, MD 20899

M. J. Mayfield

DOE

Morgantown Energy Technology Center

P.O. Box 880

Morgantown, WV 26505

G. V. McGurl

DOE

Pittsburgh Energy Technology Center

P.O. Box 10940

Pittsburgh, PA 15236

D. R. Messier

Army Materials Technology Laboratory

SLCMT-MCC

Watertown, MA 02172-0001 
T. Mulholland

Lava Crucible-Refractories Co.

P.O. Box 278

Zelienople, PA 16063

H. Narita

New Energy \& Industrial Technology

Development

Sunshine 60 Building

P.O. Box 1151

1-1 Higashi-Ikebukuro 3-Chrome

Toshima-Ku, Tokyo, 170

Japan

B. North

Kennametal, Inc.

Philip McKenna Laboratory

1011 Old Salem Road

P.O. Box 639

Greensburg, PA 15601

J. E. Notestein

DOE

Morgantown Energy Technology Center

P.O. Box 880

Morgantown, WV 26505

J. Oakey

British Coal Corporation

Coal Research Establishment

Stoke Orchard, Cheltenham

Glocestershire, England GL52 4RZ

T. E. O'Hare

Brookhaven National Laboratory

Department of Applied Science

Upton, Long Island, NY 11973

R. J. Pollina

AVCO Research Laboratory

2385 Revere Beach Parkway

Everett, MA 02149

M. Prager

The Materials Properties Council, Inc.

United Engineering Center

345 E. Forty-Seventh Street

New York, NY 10017
K. M. Prewo

United Technologies Research Center

MS 24, Silver Lane

East Hartford, CT 06108

B. H. Rabin

Idaho National Engineering Laboratory

P.O. Box 1625

Idaho Falls, ID 83415

K. L. Reifsnider

Virginia Polytechnic Institute \& State University

Department of Materials Engineering

Blackburg, VA 24601

R. Winston Revic

Canada Center for Mineral

\& Energy Technology

568 Booth Street

Ottawa, Ontario

Canada K1A OG1

S. L. Richlen

U.S. Department of Energy

Office of Industrial Technologies

1000 Independence Avenue, S.W.

Washington, D.C. 20585

Mahi Sahoo

Canada Center For Mineral

\& Energy Technology

568 Booth Street

Ottawa, Ontario

Canada K1A OG1

R. Santore

DOE

Pittsburgh Energy Technology Center

P.O. Box 10940

Pittsburgh, PA 15236

A. G. Sault

Sandia National Laboratories

Department 6211, MS 0710

Albuquerque, NM 87185 
R. B. Schulz

DOE

Office of Vehicle and Energy R\&D

CE-151 Forrestal Building

Washington, DC 20585

J. E. Scott

British Gas Corporation

Westfield Development Center

Cardenden, Fife

Scotland KY50HP

V. K. Sethi

Western Research Institute

365 N. 9th Street

P.O. Box 3395

University Station

Laramie, WY 82071

T. W. Sigmon

Research Triangle Institute

P.O. Box 12194

Research Triangle Park, NC 27709

\section{J. P. Singh}

Argonne National Laboratory

9700 S. Cass Avenue

Argonne, IL 60439

\section{S. C. Singhal}

Westinghouse Electric Corporation

Research and Development Center 1310 Beulah Road

Pittsburgh, PA 15235

\section{T. L. Starr}

Georgia Institute of Technology

Materials Science \& Engineering (0245)

Bunger-Henry Building, Room 276

Atlanta, GA 30332-0245

W. A. Steele

Lawrence Livermore Laboratory

P.O. Box 808, L-325

Livermore, CA 94550
J. Steibel

GE Aircraft Engines

One Neumann Way, MD H85

Cincinnati, OH 45215-6301

C. Steven

Williams International 2280 West Maple Raod

P.O. Box 200

Walled Lake, MI 48088

D. P. Stinton

Oak Ridge National Laboratory

P.O. Bux 2008

Oak Ridge, TN 37831

T. G. Stoebe

University of Washington

Department of Materials Science and Engineering

101 Wilson, FB-10

Seattle, WA 98195

J. Stringer

Electric Power Research Institute

P.O. Box 10412

3412 Hillview Avenue

Palo Alto, CA 94303

P. T. Thornton

Oak Ridge National Laboratory

P.O. Box 2008

Oak Ridge, TN 37831

T. M. Torkos

DOE

Pittsburgh Energy Technology Center

P.O. Box 10940

Pittsburgh, PA 15236

W. Troha

NASA

Lewis Research Center

21000 Brookpark Road

Cleveland, $\mathrm{OH} 44135$ 
A. Twigg

British Coal Corporation

Coal Research Establishment

Stoke Orchard, Cheltenham

Glochester, England GL52 4RZ

S. Ueda

New Energy \& Industrial Technology

Development

Sunshine 60 Building

P.O. Box 1151

1-1 Higashi-Tkebukuro 3-Chrome

Toshima-Ku, Tokyo, 170

Jap.n

A. W. Urquhart

Lanxide Corporation

1300 Marrows Road

P.O. Box 6077

Newark, DE 19714-6077

D. Utah

General Electric

P.O. Box 156301

Cincinnati, OH 45215-6301

M. Van de Voorde

European Communities Joint Research Centre

Petten Establishment

P.O. Box 2

1755 ZG Petten

The Netherlands

S. C. Weiner

Air Products and Chemicals

P.O. Box 538

Allentown, PA 18105

R. A. Wenglarz (Speed Code W-16)

Allison Gas Turbine Division

P.O. Box 420

Indianapolis, IN 46206-0420

\section{J. S. Wilson}

DOE, Morgantown Energy

Technology Center

P.O. Box 880

Morgantown, WV 26505
K. M. Zwilsky

National Materials Advisory Board

National Research Council 2101 Constitution Avenue Washington, DC 20418 


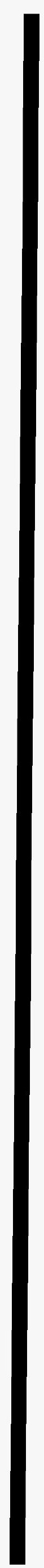

